\title{
Massive Supermultiplets in Four-Dimensional Superstring Theory
}

\author{
Wan-Zhe Fenga ${ }^{\mathrm{a}, \mathrm{b}}$, Dieter Lüst ${ }^{\mathrm{b}, \mathrm{c}}$, Oliver Schlotterer ${ }^{\mathrm{b}, \mathrm{d}}$ \\ November 1, 2018 \\ a Department of Physics, Northeastern University, Boston, MA 02115, USA \\ ${ }^{\mathrm{b}}$ Max-Planck-Institut für Physik Werner-Heisenberg-Institut, 80805 München, Germany \\ 'Arnold-Sommerfeld-Center for Theoretical Physics, \\ Ludwig-Maximilians-Universität, 80333 München, Germany \\ d Max-Planck-Institut für Gravitationsphysik Albert-Einstein-Institut, 14476 Potsdam, Germany
}

\begin{abstract}
We extend the discussion of [1] on massive Regge excitations on the first mass level of four-dimensional superstring theory. For the lightest massive modes of the open string sector, universal supermultiplets common to all four-dimensional compactifications with $\mathcal{N}=1,2$ and $\mathcal{N}=4$ spacetime supersymmetry are constructed respectively - both their vertex operators and their supersymmetry variations. Massive spinor helicity methods shed light on the interplay between individual polarization states.
\end{abstract}

LMU-ASC 10/12

MPP-2012-11

AEI-2012-018 


\section{Contents}

1 Introduction $\quad 4$

2 The first mass level in $D=10 \quad 7$

2.1 The general method . . . . . . . . . . . . . . . . . . . . . . 7

2.2 The NS sector . . . . . . . . . . . . . . . . . . . . . 8

2.3 Excited spin fields . . . . . . . . . . . . . . . . . . . . . 9

2.4 The operator content of the $\mathrm{R}$ sector . . . . . . . . . . . . . . . . . . 10

2.5 Ten-dimensional SUSY transformations . . . . . . . . . . . . . . . . . . . 11

3 CFTs of supersymmetric string vacua in four dimensions $\quad 12$

3.1 The $D=4$ spacetime supersymmetry algebra . . . . . . . . . . . . . . . . . 12

3.2 CFT realization of extended $D=4$ SUSY . . . . . . . . . . . . . . . . . . 14

3.3 CFT operators in $\mathcal{N}=4$ compactifications . . . . . . . . . . . . . . . . 15

3.4 CFT operators in $\mathcal{N}=1$ compactifications . . . . . . . . . . . . . . . . . . . . 18

3.5 CFT operators in $\mathcal{N}=2$ compactifications . . . . . . . . . . . . . . . . . . . . . 20

3.6 Summary of CFT operators . . . . . . . . . . . . . . . . . . 24

4 Massive supermultiplets for $\mathcal{N}=4 \mathrm{SUSY} \quad[24$

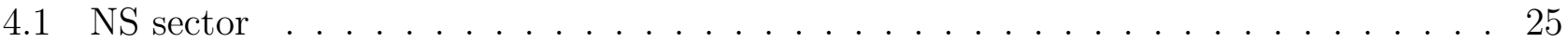

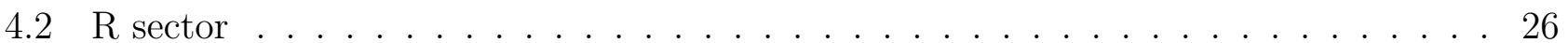

4.3 SUSY transformations . . . . . . . . . . . . . . . . . . . . . . . 28

5 Massive supermultiplets for $\mathcal{N}=1$ SUSY 34

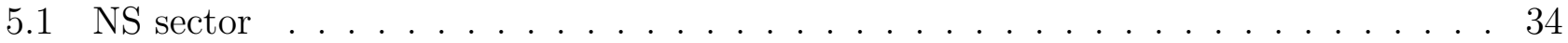

5.2 R sector ........................... . . . . . . . . . . . . .

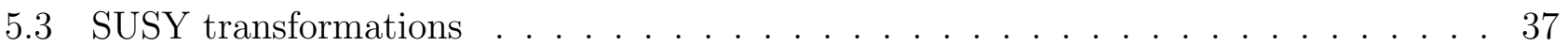

6 Massive supermultiplets for $\mathcal{N}=2$ SUSY

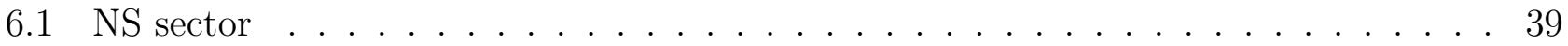

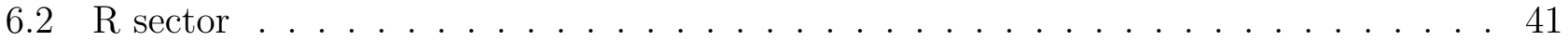

6.3 SUSY transformations . . . . . . . . . . . . . . . . . . . . . . . 42 
7 Helicity structure of massive on-shell multiplets

$7.1 \mathcal{N}=1$ supermultiplets . . . . . . . . . . . . . . . . . . 49

$7.2 \mathcal{N}=2$ supermultiplets . . . . . . . . . . . . . . . . . . 52

$7.3 \mathcal{N}=4$ supermultiplet . . . . . . . . . . . . . . . . . 56

8 Conclusions \& Outlook $\quad 60$

A Notation and convention $\quad 62$

B Operator product expansions 63

B.1 Spacetime CFT in $D=10 \ldots \ldots \ldots \ldots$. . . . . . . . . . . . . 64

B.2 Spacetime CFT in $D=4 \ldots \ldots \ldots \ldots \ldots$

B.3 Internal CFT for $\mathcal{N}=4 \mathrm{SUSY} \ldots \ldots \ldots \ldots$

B.4 Internal CFT for $\mathcal{N}=1 \mathrm{SUSY} \ldots \ldots \ldots \ldots$

C Spinor helicity methods for massive wavefunctions 68

C.1 Massive spin one boson . . . . . . . . . . . . . . . . . . . . . . . . 69

C.2 Massive spin two boson . . . . . . . . . . . . . . . . . . . . . 70

C.3 Massive spin $1 / 2$ fermions . . . . . . . . . . . . . . . . . . . . . . . . 70 70

C.4 Massive spin $3 / 2$ fermions . . . . . . . . . . . . . . . . . . 71 


\section{Introduction}

String theory as seen from the point particle perspective contains an infinite number of massive, higher spin states. As known already from the early days of string theory, these massive states lie on the so-called Regge trajectories that display the linear relation between the (mass) ${ }^{2}$ and the spin $J$ of all states. The existence of the infinitely many higher spin states is essential for the ultra-violet behavior of string scattering amplitudes. It unitarizes all string amplitudes among the massless modes in the UV via the exchange of the infinite tower of massive states, as it was first shown in the famous Veneziano amplitude that describes the scattering of four massless open string states. In addition the consistency of quantum gravity in string theory completely relies on the massive higher spin states. Since the size of a string grows with its excitation energy, larger and larger states are produced at higher and higher energies. Hence, the UV properties of string scattering amplitudes are non-Wilsonian, which is also manifest in the UV-IR mixing in string theory. As it was argued in [2] this might lead to a reformulation of the Heisenberg uncertainty principle in string theory with the result that the string scale appears at the shortest possible length scale, which can be dissolved in string scattering experiments.

The existence of massive higher spin states in string theory is not only crucial for the consistency of the theory, but is possibly also interesting from the phenomenological point of view. Since

the masses of the higher spin states are all multiples of the string scale $M_{s}=\sqrt{\alpha^{\prime-1}}$, D-brane compactifications with a low string scale $M_{s}$ in the TeV region and with large extra dimensions offer the exciting possibility that the lightest Regge excitations of massless open strings can be directly produced and detected at the LHC. As it was shown in [3, 4], four- and five-point string scattering amplitudes among standard model gauge bosons (gluons, $W, Z$-bosons, photons) and at most two external massless fermions (quarks or leptons) are completely independent from any geometrical details of the underlying D-brane model in four dimensions. Hence, the production of the first heavy colored string states (e.g. excited gluons with $J=0,2$ ) from gluon fusion and their subsequent decay into two or three hadronic jets leads to completely model independent cross sections and decay rates at the LHC. Based on these calculations the recent LHC searches for non-standard dijet events due to heavy new resonances can now exclude massive string states with masses below about $4 \mathrm{TeV}$.

This paper is not so much concerned about the phenomenological implications of massive higher spins states, but we rather like to exploit some of the basic supersymmetry properties of higher spin 
states in four dimensions, originating from supersymmetric type II compactifications. In [1] we already computed string scattering amplitudes not only with massless external string states, but also three- and four-point amplitudes with one massive excited open string state as external field. For this purpose we constructed in [1] the corresponding covariant vertex operators for the lowest massive open states in four dimensions $1^{1}$ focusing in particular on those universal open Regge states, which are present in any D-brane compactification to four dimensions (excited gluons and gluinos). Working within the world-sheet NS-R formalism, physical, massive states belong to the cohomology of the BRST operator. In addition, besides world-sheet conformal invariance, supersymmetry plays a key role for the consistency of string theory, both on the world-sheet as well as in target space. In ten spacetime dimensions, the type $\operatorname{IIB}(\mathrm{A})$ superstring exhibits extended (non-) chiral $\mathcal{N}=2$ spacetime supersymmetry with in total 32 supersymmetry charges. It follows that all massless as well as all massive closed string states are organized in supermultiplets of the ten-dimensional $\mathcal{N}=2$ supersymmetry algebra. This leads to a very subtle interplay between massive string excitations with different higher spins that belong to common supersymmetry multiplets. In fact, the covariant world-sheet vertex operators of the higher spin states must transform into each other when acting on them with the supersymmetry charge operators. Hence, spacetime supersymmetry must be reflected in the structure of the world-sheet BRST cohomology on each mass level of the higher spin excitations.

Going from ten to lower dimensions, parts or all of spacetime supersymmetry can be preserved during the compactification process. As it is known already for several years [11, 12, 13, 14], there exists a deep relation between the number of spacetime supersymmetries, preserved by the compactification, and the number of world-sheet supersymmetries of the corresponding internal superconformal field theory. Specifically, for type II compactifications on six-dimensional CalabiYau spaces, which correspond to $\hat{c}=6$ SCFT's with $(2,2)$ world sheet supersymmetry, one obtains in the closed string sector four-dimensional $\mathcal{N}=2$ effective supergravity theories with 8 preserved supercharges in the bulk. Second, type II compactification on $K 3 \times T^{2}$ with four-dimensional $\mathcal{N}=4$ spacetime supersymmetry (16 bulk supercharges) can be described by the direct product of two SCFT's with central charges $\hat{c}=4$ and $\hat{c}=2$, where the $\hat{c}=4$ part possesses $(4,4)$ supersymmetry on the world-sheet. Finally, compactifications on a six-dimensional torus leads to effective type II supergravity theories with maximal $\mathcal{N}=8$ supersymmetry (32 bulk supercharges).

However, when also including D-branes and open strings, the number of spacetime supersym-

\footnotetext{
${ }^{1}$ Additional and previous work on vertex operators for massive higher spin excitations includes [5, 6, 7, 8, 9, 10.
} 
metries is reduced by half compared to the closed string bulk sector, we just discussed above. First, the effective, four-dimensional Yang-Mills theories of type IIB, Calabi-Yau orientifolds with D3/D7branes or with D5/D9-branes (or type IIA Calabi-Yau orientifolds with intersecting D6-branes) possess just $\mathcal{N}=1$ supersymmetry. Next the IIB $K 3 \times T^{2}$ orientifolds with D5/D9-branes lead to $\mathcal{N}=2$ supersymmetric Yang-Mills theories in four dimensions..$^{2}$ And finally, toroidal compactifications of type II superstrings lead to Yang-Mills open string sectors with $\mathcal{N}=4$ supersymmetry in $D=4$.

It is the aim of this paper to extend the work of [1] in order to systematically construct the covariant vertex operators of the lowest massive open string supermultiplets for all three cases of $\mathcal{N}=4,2,1$ spacetime supersymmetry on the corresponding D-branes. We will focus in particular on those massive supermultiplets and their SUSY transformations in the universal sector, which are always present in any four-dimensional orientifold models:

- For $\mathcal{N}=4$ super Yang-Mills, there is a single massive, spin two supermultiplet with 128 bosonic as well as 128 fermionic degrees of freedom.

- The supermultiplets of the universal $\mathcal{N}=1$ sector contains one spin two supermultiplet and two spin $1 / 2$ representations with in total $12+12$ bosonic and fermion degrees of freedom.

- Finally, for $\mathcal{N}=2$ super Yang-Mills we are dealing with $40+40$ massive open string states, being organized in one spin two plus two spin one massive supermultiplets.

In this way we extend the analysis of [14] about the relation between world-sheet and spacetime supersymmetries and their closed string (massless) supermultiplet structure to the case of the massive, open string supermultiplets. At the same time we are giving here a massive version of the SUSY multiplet analysis in [15], where it was shown that SUSY Ward identities among scattering amplitudes are valid to all orders in $\alpha^{\prime}$, and where the spinor helicity methods were applied to make efficient use of these Ward identities.

The paper is organized as follows. As a warm-up case, in section 2 we first construct the covariant NS and R vertex operators of the ten-dimensional type I open string states at the first mass level. They comprise in total $128+128$ bosonic as well as fermionic states. We verify that these states form a massive representation of the ten-dimensional (type I) $\mathcal{N}=1$ SUSY algebra. Next, in section 3 we consider the SCFT's of string vacua in four dimensions, and discuss the

\footnotetext{
${ }^{2}$ These theories originate upon compactification on $T^{2}$ from $D=6$, IIB theories on $K 3$ with $(1,1)$ spacetime supersymmetry.
} 
relation between the extended world-sheet superconformal algebras and the spacetime $\mathcal{N}=4,1,2$ SUSY algebras and the covariant vertex operators for the corresponding supercharge operators. Sections 4, 5 and 6 are devoted to construct the massive open string supermultiplets, their vertex operators and their supersymmetry transformations for the three cases of $\mathcal{N}=4, \mathcal{N}=1$ and $\mathcal{N}=2$ supersymmetry in four dimensions respectively. Finally, in section 7 we study in more detail in helicity structure of the various on-shell supermultiplets.

\section{The first mass level in $D=10$}

The lightest Regge excitations of open superstring theory in ten-dimensional Minkowski spacetime were firstly constructed in 1987 [5]. Let us briefly review the general method to construct heavy string excitations as well as the explicit results of [5] and then offer a covariant approach to the excited Ramond sector states.

\subsection{The general method}

Physical states belong to the cohomology of the BRST operator $Q_{\mathrm{BRST}}$. In the world-sheet variables of the RNS formalism, it splits into three pieces of different superghost charge:

$$
\begin{aligned}
Q_{\mathrm{BRST}} & =Q_{0}+Q_{1}+Q_{2}, \\
Q_{0} & =\oint \frac{\mathrm{d} z}{2 \pi i}\left(c\left(T+T_{\beta, \gamma}\right)+b c \partial c\right), \\
Q_{1} & =-\oint \frac{\mathrm{d} z}{2 \pi i} \gamma G=-\oint \frac{\mathrm{d} z}{2 \pi i} \mathrm{e}^{\phi} \eta G, \\
Q_{2} & =-\frac{1}{4} \oint \frac{\mathrm{d} z}{2 \pi i} b \gamma^{2}=-\frac{1}{4} \oint \frac{\mathrm{d} z}{2 \pi i} b \mathrm{e}^{2 \phi} \eta \partial \eta .
\end{aligned}
$$

We denote the $c=15$ stress tensor and supercurrent of the matter fields ${ }^{3} i \partial X^{m}, \psi^{n}$ by $T$ and $G$, respectively, whereas $T_{\beta, \gamma}$ captures the $\beta, \gamma$ superghost system of $c=11$. The latter is partially bosonized in terms of exponentials $\mathrm{e}^{q \phi}$ (with $\phi$ denoting a free chiral boson) and completed by a pair of $h=1,0$ fermions $\eta, \xi$. The Grassmann odd ghost system $(b, c)$ is well-known from the bosonic string.

\footnotetext{
${ }^{3}$ Our normalization conventions for the world-sheet matter fields are fixed by

$$
i \partial X^{m}(z) i \partial X^{n}(w) \sim \frac{2 \alpha^{\prime} \eta^{m n}}{(z-w)^{2}}+\ldots, \quad \psi^{m}(z) \psi^{n}(w) \sim \frac{\eta^{m n}}{z-w}+\ldots .
$$
}


States of uniform superghost charge are BRST closed only if they are annihilated by $Q_{0}, Q_{1}$ and $Q_{2}$ separately. Closure under $Q_{0}$ forces vertex operators to be a Virasoro primary of unit weight, while $Q_{2}$ does not contribute in the ghost pictures considered in this paper. Hence, given a vertex operator ansatz of suitable conformal weight, only the $Q_{1}$ constraint involving the supercurrent

$$
G(z)=\frac{1}{2 \sqrt{2 \alpha^{\prime}}} i \partial X_{m}(z) \psi^{m}(z)
$$

has to be evaluated separately.

\subsection{The NS sector}

The lowest mass $m^{2}=-k^{2}=1 / \alpha^{\prime}$ for Regge excitations assigns conformal weight $h=-1$ to the plane wave $\mathrm{e}^{i k \cdot X}$ which introduces spacetime momentum into vertex operators. In the NS sector of canonical superghost charge -1 , it can combine with the $h=\frac{1}{2}$ field $\mathrm{e}^{-\phi}$ and a $h=\frac{3}{2}$ combination of $i \partial X^{m}, \psi^{n}$ oscillators to form a Virasoro primary of unit conformal weight in total. (Hence, neglecting the plane wave $\mathrm{e}^{i k \cdot X}$ contribution, the massive states at first mass level always correspond to vertex operators with conformal dimension $h=2$.)

The most general $h=1$ ansatz for the first massive NS sector states involves thre $4^{4} h=\frac{3}{2}$ operators $i \partial X^{m} \psi^{n}, \psi^{m} \psi^{n} \psi^{p}$ and $\partial \psi^{m}$ along with polarization wavefunctions $B_{m n}, E_{m n p}, H_{m}$ :

$$
V^{(-1)}(B, E, H, k, z)=\left(B_{m n} i \partial X^{m} \psi^{n}+E_{m n p} \psi^{m} \psi^{n} \psi^{p}+H_{m} \partial \psi^{m}\right) \mathrm{e}^{-\phi} \mathrm{e}^{i k \cdot X}
$$

The BRST constraints arising from $Q_{1}$ admit two physical solutions ${ }^{5}$ namely a (traceless and symmetric) spin two tensor $B_{m n}$ and a three-form $E_{m n p}$ :

$$
\begin{aligned}
V^{(-1)}(B, k, z) & =\frac{1}{\sqrt{2 \alpha^{\prime}}} B_{m n} i \partial X^{m} \psi^{n} \mathrm{e}^{-\phi} \mathrm{e}^{i k \cdot X}, \quad k^{m} B_{m n}=B_{m}{ }^{m}=B_{[m n]}=0, \\
V^{(-1)}(E, k, z) & =\frac{1}{6} E_{m n p} \psi^{m} \psi^{n} \psi^{p} \mathrm{e}^{-\phi} \mathrm{e}^{i k \cdot X}, \quad k^{m} E_{m n p}=0 .
\end{aligned}
$$

Both polarizations are transverse and therefore naturally fall into representations of the stabilizer group $S O(9)$ of massive momenta. The number of degrees of freedom is $\frac{9 \cdot 10}{2}-1=44$ for $B_{m n}$ and $\frac{9 \cdot 8 \cdot 7}{1 \cdot 2 \cdot 3}=84$ for $E_{m n p}$, i.e. we have $44+84=128$ bosonic states in total.

\footnotetext{
${ }^{4}$ The addition of $\xi_{m} \psi^{m} \partial \phi \mathrm{e}^{-\phi}$ is neglected because it can be absorbed into a total derivative.

${ }^{5}$ Throughout this paper, we are setting the vertex operator normalization factor $g_{\mathrm{A}}=\sqrt{2 \alpha^{\prime}} g_{\mathrm{YM}}$ from $[3$, 4, to unity.
} 
Some of the solutions to the BRST constraint turn out to be $Q_{\mathrm{BRST}}$ exact:

$$
\begin{aligned}
{\left[Q_{\mathrm{BRST}}, \mathrm{e}^{-2 \phi} \Sigma_{[m n]} \psi^{m} \psi^{n} \partial \xi \mathrm{e}^{i k \cdot X}\right] } & \sim\left(2 \Sigma_{[m n]} i \partial X^{m} \psi^{n}+\Sigma_{[m n} k_{p]} \psi^{m} \psi^{n} \psi^{p}\right) \mathrm{e}^{-\phi} \mathrm{e}^{i k \cdot X} \\
{\left[Q_{\mathrm{BRST}}, \mathrm{e}^{-2 \phi} \pi_{m} i \partial X^{m} \partial \xi \mathrm{e}^{i k \cdot X}\right] } & \sim\left(\pi_{m} \partial \psi^{m}+\pi_{m} k_{n} i \partial X^{m} \psi^{n}\right) \mathrm{e}^{-\phi} \mathrm{e}^{i k \cdot X}, \\
{\left[Q_{\mathrm{BRST}}, \partial \mathrm{e}^{-2 \phi} \partial \xi \mathrm{e}^{i k \cdot X}\right] } & \sim\left(\left[\frac{\eta_{m n}}{2 \alpha^{\prime}}+2 k_{m} k_{n}\right] i \partial X^{m} \psi^{n}+3 k_{m} \partial \psi^{m}\right) \mathrm{e}^{-\phi} \mathrm{e}^{i k \cdot X}
\end{aligned}
$$

These spurious states parametrized by a two-form $\Sigma_{[m n]}$, a vector $\pi_{m}$ and a scalar of $S O(9)$ (i.e. subject to $k^{m} \Sigma_{m n}=k^{m} \pi_{m}=0$ ) decouple from physical states.

\subsection{Excited spin fields}

In the $\mathrm{R}$ sector, the canonical superghost vacuum is created by the $h=\frac{3}{8}$ field $\mathrm{e}^{-\phi / 2}$. Masses $m^{2}=1 / \alpha^{\prime}$ allow for an $h=\frac{13}{8}$ operator to complete fermionic vertex operators for the first mass level. The matter sector of the $\mathrm{R}$ ground states corresponds to $h=\frac{5}{8}$ spin fields $S_{\alpha}$ transforming as left-handed spinors of the Lorentz group [16, 17]. The right-handed chirality is forbidden by GSO projection. The role of $S_{\alpha}$ to open or close branch cuts for the $\psi^{m}$ is reflected in the OPE

$$
\psi^{m}(z) S_{\alpha}(w) \sim \frac{\gamma_{\alpha \dot{\beta}}^{m}}{\sqrt{2}(z-w)^{1 / 2}} S^{\dot{\beta}}(w)+\ldots .
$$

The nontrivial three-point interactions between $\psi^{m}$ and $S_{\alpha}$ render their covariant correlation functions inaccessible to the Wick theorem, one has to use techniques of [18, 19, 20] instead to compute higher order correlators. Only by breaking $S O(1,9)$ to its $S U(5)$ subgroup, one can relate the $\psi^{m}$ and $S_{\alpha}$ to a free field system of chiral bosons $H_{1,2, \ldots, 5}$ :

$$
i \partial H_{k}(z) i \partial H_{l}(w) \sim \frac{\delta_{k l}}{(z-w)^{2}}+i \partial H_{k}(w) i \partial H_{l}(w)+\ldots
$$

This technique is known as bosonization $[$ [17]:

$$
\psi^{m} \leftrightarrow \mathrm{e}^{ \pm i H_{m}}, \quad S_{\alpha} \leftrightarrow \mathrm{e}^{ \pm i H_{1} / 2} \mathrm{e}^{ \pm i H_{2} / 2} \mathrm{e}^{ \pm i H_{3} / 2} \mathrm{e}^{ \pm i H_{4} / 2} \mathrm{e}^{ \pm i H_{5} / 2}
$$

It is clear from this bosonized representation that the subleading term $\sim(z-w)^{1 / 2}$ of the OPE (2.11) involves $\mathrm{e}^{ \pm 3 i H_{k} / 2}$ primary operators, in addition to the derivatives $\partial \mathrm{e}^{ \pm i H_{k} / 2}$. The covariant

\footnotetext{
${ }^{6}$ We should admit that our discussion neglects Jordan-Wigner cocycle factors [17. These are additional algebraic objects accompanying the exponentials to ensure that $\mathrm{e}^{ \pm i H_{k}}$ and $\mathrm{e}^{ \pm i H_{l}}$ associated with different bosons $k \neq l$ anticommute. We drop cocycle factors to simplify the notation, it suffices to remember that they are implicitly present and that the bosonized representation of $\psi^{\mu}$ still obeys Fermi statistics. The instance where they contribute a phase is commented on above 6.39 .
} 
description of these new excited primary fields requires an irreducible vector spinor

$$
S_{m}^{\dot{\beta}} \leftrightarrow \quad \mathrm{e}^{ \pm i 3 H_{1} / 2} \mathrm{e}^{ \pm i H_{2} / 2} \mathrm{e}^{ \pm i H_{3} / 2} \mathrm{e}^{ \pm i H_{4} / 2} \mathrm{e}^{ \pm i H_{5} / 2}, \quad \gamma_{\alpha \dot{\beta}}^{m} S_{m}^{\dot{\beta}}=0
$$

of weight $h=\frac{13}{8}$, where the gamma tracelessness condition subtracts the descendant components $\partial S_{\alpha} \leftrightarrow \partial\left(\mathrm{e}^{ \pm i H_{1} / 2} \mathrm{e}^{ \pm i H_{2} / 2} \mathrm{e}^{ \pm i H_{3} / 2} \mathrm{e}^{ \pm i H_{4} / 2} \mathrm{e}^{ \pm i H_{5} / 2}\right)$. The introduction of $S_{m}^{\dot{\beta}}$ and $\partial S_{\alpha}$ is the covariant way to disentangle the primary field- and descendant components within the operator $\psi_{m} \psi^{n} S_{\alpha} \gamma_{n}^{\alpha \dot{\beta}}$ used in [5]. The completion of the OPE (2.11) to the subleading level reads

$$
\psi^{m}(z) S_{\alpha}(w) \sim \frac{\gamma_{\alpha \dot{\beta}}^{m} S^{\dot{\beta}}(w)}{\sqrt{2}(z-w)^{1 / 2}}+(z-w)^{1 / 2}\left[S_{\alpha}^{m}(w)+\frac{2}{\sqrt{2} 5} \gamma_{\alpha \dot{\beta}}^{m} \partial S^{\dot{\beta}}(w)\right]+\ldots
$$

in $D=10$. A more exhaustive list of OPEs involving $\psi^{m}, S_{\alpha}$ and $S_{m}^{\dot{\beta}}$ (and their counterparts of opposite $S O(1,9)$ chirality) can be found in appendix B.1. A covariant treatment of generic higher spin primary fields will be given in [21].

\subsection{The operator content of the $\mathrm{R}$ sector}

After the GSO projection, the most general vertex operator for spacetime fermions at the first mass level involves the $h=\frac{13}{8}$ operators $i \partial X^{m} S_{\alpha}, S_{m}^{\dot{\beta}}$ and $\partial S_{\alpha}$ and therefore two vector spinor wavefunctions $v_{m}^{\alpha}, \bar{\rho}_{\dot{\beta}}^{m}$ as well as spinor wavefunction $u^{\alpha}$ :

$$
V^{(-1 / 2)}(v, \bar{\rho}, u, k, z)=\left(v_{m}^{\alpha} i \partial X^{m} S_{\alpha}+\bar{\rho}_{\dot{\beta}}^{m} S_{m}^{\dot{\beta}}+u^{\alpha} \partial S_{\alpha}\right) \mathrm{e}^{-\phi / 2} \mathrm{e}^{i k \cdot X}
$$

Since $\bar{\rho}$ is contracted with the excited spin field $S_{m}^{\dot{\beta}}$, we can regard it as $\gamma$ traceless, i.e. $\bar{\rho}_{\dot{\beta}}^{m} \bar{\gamma}_{m}^{\dot{\beta} \alpha}=0$. The independent $Q_{1}$ BRST constraints for 2.16 can be summarized as

$$
\begin{aligned}
& 0=2 \alpha^{\prime} v_{m}^{\alpha} \not k_{\alpha \dot{\beta}}+\sqrt{2} \bar{\rho}_{m, \dot{\beta}}+\frac{1}{2} u^{\alpha} \gamma_{m \alpha \dot{\beta}}, \\
& 0=2 \sqrt{2} k_{\mu} \bar{\rho}_{\dot{\beta}}^{\mu}-\frac{3}{2} u^{\alpha} \not k_{\alpha \dot{\beta}} .
\end{aligned}
$$

Disentangling the $S O(1,9)$ irreducibles of the former allows to express $u^{\alpha}$ and $\bar{\rho}_{\dot{\beta}}^{m}$ in terms of $v_{m}^{\alpha}$,

$$
\begin{aligned}
\bar{\rho}_{\dot{\beta}}^{m} & =-\sqrt{2} \alpha^{\prime}\left(v^{m \alpha} \not k_{\alpha \dot{\beta}}+\frac{1}{10} v_{p}^{\alpha}\left(\not k \bar{\gamma}^{p} \gamma^{m}\right)_{\alpha \dot{\beta}}\right), \\
u^{\alpha} & =\frac{2 \alpha^{\prime}}{5} v_{m}^{\beta}\left(\not k \gamma^{m}\right)_{\beta}^{\alpha},
\end{aligned}
$$

whereas 2.18 yields an extra constraint on the only independent polarization $v_{m}^{\alpha}$ :

$$
v_{m}^{\alpha} \gamma_{\alpha \dot{\beta}}^{m}=2 \alpha^{\prime} k^{m} v_{m}^{\alpha} \not k_{\alpha \dot{\beta}}
$$


As recognized in [5], there is a physical solution $v_{m}^{\alpha} \equiv \chi_{m}^{\alpha}$ of spin $3 / 2$

$$
\begin{aligned}
V^{(-1 / 2)}(\chi, k) & =\frac{1}{\sqrt{2} \alpha^{1 / 4}}\left(\chi_{m}^{\alpha} i \partial X^{m} S_{\alpha}-\sqrt{2} \alpha^{\prime} \chi^{m \alpha} \not k_{\alpha \dot{\beta}} S_{m}^{\dot{\beta}}\right) \mathrm{e}^{-\phi / 2} \mathrm{e}^{i k \cdot X} \\
0 & =k^{m} \chi_{m}^{\alpha}=\chi_{m}^{\alpha} \gamma_{\alpha \dot{\beta}}^{m}
\end{aligned}
$$

and one spurious state associated with the gamma trace choice $v_{m}^{\alpha}=k_{m} \Theta^{\alpha}+\frac{1}{4} \Theta^{\beta}\left(\not k \gamma_{m}\right)_{\beta}^{\alpha}$

$$
\begin{aligned}
{\left[Q_{\text {BRST }}, \mathrm{e}^{-3 \phi / 2} \partial \xi \Theta^{\alpha} \not k_{\alpha \dot{\beta}} S^{\dot{\beta}} \mathrm{e}^{i k \cdot X}\right] \sim\left(\left[k_{m} \Theta^{\alpha}+\frac{1}{4} \Theta^{\beta}\left(\not k \gamma_{m}\right)_{\beta}{ }^{\alpha}\right] i \partial X^{m} S_{\alpha}\right.} \\
\left.-\frac{1}{\sqrt{2}}\left[\alpha^{\prime} k^{m} \Theta^{\alpha} \not k_{\alpha \dot{\beta}}+\frac{1}{10} \Theta^{\alpha} \gamma_{\alpha \dot{\beta}}^{m}\right] S_{m}^{\dot{\beta}}+\frac{6}{5} \Theta^{\alpha} \partial S_{\alpha}\right) \mathrm{e}^{-\phi / 2} \mathrm{e}^{i k \cdot X}
\end{aligned}
$$

which allows to gauge away the $u^{\alpha}$ wavefunction.

\subsection{Ten-dimensional SUSY transformations}

The SUSY charge in open superstring theory is given by the massless gaugino vertex at zero momentum [11]:

$$
\mathcal{Q}_{\alpha}^{(-1 / 2)}=\frac{1}{\alpha^{\prime 1 / 4}} \oint \frac{\mathrm{d} z}{2 \pi i} S_{\alpha} \mathrm{e}^{-\phi / 2}
$$

It transforms $\mathrm{R}$ sector states in their canonical $-1 / 2$ superghost picture into canonical NS vertex operators $\sim \mathrm{e}^{-\phi}$. The contour integral is evaluated by performing OPEs between the $S_{\alpha}$ and $\mathrm{e}^{-\phi / 2}$ fields from the supercharge at point $z$ and the vertex operator $V^{(-1 / 2)}(w)$ of the fermion in question. Appendix B.1 gathers the required OPEs for the $D=10$ case.

The inverse transformation from the NS sector to the $\mathrm{R}$ sector requires the $+1 / 2$ picture representative of the SUSY generator

$$
\mathcal{Q}_{\alpha}^{(+1 / 2)}=\frac{1}{2 \alpha^{\prime 3 / 4}} \oint \frac{\mathrm{d} z}{2 \pi i} i \partial X_{m} \gamma_{\alpha \dot{\beta}}^{m} S^{\dot{\beta}} \mathrm{e}^{+\phi / 2}
$$

The latter allows to write down the ghost neutral $\mathcal{N}=1$ SUSY algebra in ten dimensions,

$$
\left\{\mathcal{Q}_{\alpha}^{(+1 / 2)}, \mathcal{Q}_{\beta}^{(-1 / 2)}\right\}=\left(\gamma^{m} C\right)_{\alpha \beta} P_{m}, \quad P_{m}=\frac{1}{2 \alpha^{\prime}} \oint \frac{\mathrm{d} z}{2 \pi i} i \partial X_{m}
$$

Let us list the SUSY variations of the physical $D=10$ vertex operators. The NS sector states (2.8) and 2.9 have already been discussed in [5]

$$
\begin{aligned}
& {\left[\eta^{\alpha} \mathcal{Q}_{\alpha}^{(+1 / 2)}, V^{(-1)}(B, k)\right]=V^{(-1 / 2)}\left(\chi_{m}^{\alpha}=\frac{1}{\sqrt{2}} B_{m n}\left(\eta \not k \gamma^{n}\right)^{\alpha}\right)} \\
& {\left[\eta^{\alpha} \mathcal{Q}_{\alpha}^{(+1 / 2)}, V^{(-1)}(E, k)\right]=V^{(-1 / 2)}\left(\chi_{m}^{\alpha}=\frac{1}{12 \sqrt{\alpha^{\prime}}}\left[E_{m n p}\left(\eta \gamma^{n p}\right)^{\alpha}-\frac{1}{3} E_{n p q}\left(\eta \gamma_{m} \gamma^{n p q}\right)^{\alpha}\right.\right.}
\end{aligned}
$$




$$
\left.\left.-\frac{\alpha^{\prime}}{3} k_{m} E_{n p q}\left(\eta \not k \gamma^{n p q}\right)^{\alpha}\right]\right)
$$

In addition, we use the covariant OPEs from appendix B.1 to compute the SUSY variation of the massive gravitino 2.22):

$$
\begin{gathered}
{\left[\eta^{\alpha} \mathcal{Q}_{\alpha}^{(-1 / 2)}, V^{(-1 / 2)}(\chi, k)\right]=V^{(-1)}\left(B_{m n}=\frac{\alpha^{\prime}}{\sqrt{2}}\left(\eta \not k \chi_{(m}\right) k_{n)}+\frac{1}{\sqrt{2}}\left(\eta \gamma_{(m} \chi_{n)}\right)\right)} \\
+V^{(-1)}\left(E_{m n p}=3 \alpha^{\prime 1 / 2}\left(\eta \gamma_{[m} \chi_{n}\right) k_{p]}-\frac{3}{2} \alpha^{\prime 1 / 2}\left(\eta \gamma_{[n p} \not k \chi_{m]}\right)\right) .
\end{gathered}
$$

\section{CFTs of supersymmetric string vacua in four dimensions}

In this section we will first review some basic facts about extended supersymmetry algebras in four spacetime dimensions and about the general relation between extended spacetime supersymmetries and world-sheet supersymmetries. In part, we are following the work in references [12, 13, 14]. Our conventions for indices w.r.t. Lorentz symmetry $S O(1,3)$ and R-symmetries $S O(6)$ or $S U(2)$ are gathered in appendix A.

\subsection{The $D=4$ spacetime supersymmetry algebra}

The $\mathcal{N}$ supercharges $\mathcal{Q}_{a}^{I}$ as well as the complex conjugate operators $\overline{\mathcal{Q}}_{\bar{I}}^{\dot{a}}$ satisfy the $\mathcal{N}$-extended supersymmetry algebra $(I, \bar{I}=1 \ldots, \mathcal{N})$

$$
\begin{aligned}
\left\{\mathcal{Q}_{a}^{I}, \overline{\mathcal{Q}}_{\bar{J}}^{\dot{b}}\right\} & =C_{\bar{J}}^{I}\left(\sigma^{\mu} \varepsilon\right)_{a}{ }^{\dot{b}} P_{\mu}, \\
\left\{\mathcal{Q}_{a}^{I}, \mathcal{Q}_{b}^{J}\right\} & =\varepsilon_{a b} \mathcal{Z}^{I J} .
\end{aligned}
$$

$P^{\mu}$ is the momentum operator and the $\mathcal{Z}^{I J}$ are central charges, which are antisymmetric in $I, J$ and can therefore appear in the $\mathcal{N} \geq 2$ supersymmetry algebra only.

Next let us discuss the representations of the extended supersymmetry algebras, namely how the supercharges in general act on massless and on massive states. Let us first recall the case of massless states. Here we can choose a frame where the momenta are $k^{\mu}=(E, 0,0, E)$, the supercharges are

$$
\mathcal{Q}_{1}^{I} \equiv \mathcal{Q}^{I}, \quad \overline{\mathcal{Q}}_{\bar{I}}^{\dot{1}} \equiv \overline{\mathcal{Q}}_{I}, \quad \text { whereas } \quad 0=\mathcal{Q}_{1}^{2}=\overline{\mathcal{Q}}_{\bar{I}}^{\dot{2}}
$$

In terms of $\mathcal{Q}^{I}$ and $\overline{\mathcal{Q}}_{I}$ the supersymmetry algebra takes the form

$$
\left\{\mathcal{Q}^{I}, \overline{\mathcal{Q}}_{J}\right\}=\delta_{J}^{I},
$$




$$
\left\{\mathcal{Q}^{I}, \mathcal{Q}^{J}\right\}=\left\{\overline{\mathcal{Q}}_{I}, \overline{\mathcal{Q}}_{J}\right\}=0
$$

where we have rescaled the supersymmetry charges by $\sqrt{E}$. The $2 \mathcal{N}$ supercharges $\mathcal{Q}^{I}$ and $\overline{\mathcal{Q}}_{I}$ build an $S O(2 \mathcal{N})$ Clifford algebra

$$
\begin{aligned}
\Gamma_{2 I-1} & =\mathcal{Q}^{I}+\overline{\mathcal{Q}}_{\bar{I}}, \quad \Gamma_{2 I}=i\left(\mathcal{Q}^{I}-\overline{\mathcal{Q}}_{\bar{I}}\right), \\
\left\{\Gamma_{i}, \Gamma_{j}\right\} & =2 \delta_{i j}, \quad i, j=1, \ldots, 2 \mathcal{N}
\end{aligned}
$$

whose representations have dimension $2^{\mathcal{N}}$. The generators for $S O(2 \mathcal{N})$ rotations are

$$
\Lambda_{i j}=\frac{1}{4 i}\left[\Gamma_{i}, \Gamma_{j}\right]
$$

This group contains a $S U(\mathcal{N}) \times U(1)$ subgroup specified by the following generators

$$
\begin{aligned}
\Lambda_{J}^{I} & =\frac{1}{2}\left[\mathcal{Q}^{I}, \overline{\mathcal{Q}}_{\bar{J}}\right]-\frac{1}{2 \mathcal{N}} \delta_{J}^{I}\left[\mathcal{Q}^{K}, \overline{\mathcal{Q}}_{\bar{K}}\right] \text { for } S U(\mathcal{N}), \\
\Lambda & =\frac{1}{4}\left[\mathcal{Q}^{I}, \overline{\mathcal{Q}}_{I}\right] \text { for } U(1) .
\end{aligned}
$$

For massless states, this $S U(\mathcal{N})$ commutes with the $S O(2)$ helicity group. Hence this group classifies massless states. The eigenvalue of the supercharge under the $U(1)$, which is called intrinsic helicity, is the same as under spacetime helicity. Therefore one can define a new generator $\Lambda^{\prime}$ through a shift by the $z$ component $j^{3}$ of the spin, called superhelicity,

$$
\Lambda^{\prime}=j^{3}-\Lambda,
$$

which commutes with $\mathcal{Q}^{I}$.

Next let us consider massive states rotated into their rest frame $k^{\mu}=(m, 0,0,0)$. Now also the second helicity components of the supercharge spinors become active, i.e. give rise to nonzero supersymmetry transformations on massive states. We will denote them as follows:

$$
\mathcal{Q}_{2}^{I} \equiv \tilde{\mathcal{Q}}^{I}, \quad \overline{\mathcal{Q}}_{\bar{I}}^{\dot{2}} \equiv \overline{\tilde{\mathcal{Q}}}_{\bar{I}}
$$

The supersymmetry algebra between the $\tilde{\mathcal{Q}}$ looks like

$$
\left\{\tilde{\mathcal{Q}}^{I}, \overline{\tilde{\mathcal{Q}}}_{\bar{J}}\right\}=m C_{\bar{J}}^{I}, \quad\left\{\tilde{\mathcal{Q}}^{I}, \tilde{\mathcal{Q}}^{J}\right\}=\left\{\overline{\tilde{\mathcal{Q}}}_{I}, \overline{\tilde{\mathcal{Q}}}_{J}\right\}=0
$$

Now the $\left(\mathcal{Q}^{I}, \bar{Q}_{\bar{I}}\right)$ and $\left(\tilde{\mathcal{Q}}^{I}, \overline{\tilde{\mathcal{Q}}}_{\bar{I}}\right)$ build an $S O(4 \mathcal{N})$ Clifford algebra on the states without central charges. Consequently, the dimension of massive representations is a multiple of $2^{2 \mathcal{N}}$. The maximal 
subalgebra that commutes with the $S O(3)$ little group of the massive states is $U S p(2 \mathcal{N})$. Therefore massive states without central charges build representations of $U S p(2 \mathcal{N})$. As for the massless states one can consider an $S U(\mathcal{N}) \times U(1)$ subgroup with generators $\Lambda_{t o t}=\Lambda+\tilde{\Lambda}$ where the $\tilde{\Lambda}$ are defined from the $\tilde{\mathcal{Q}}$ as in 3.6 . In section 7 we will introduce an organization scheme for massive SUSY representations based on spinor helicity methods which keeps track of the spin quantum numbers along a reference axis of choice.

However, in the presence of central charges $\mathcal{Z}^{I J}$, the operators $\mathcal{Q}^{I}$ and $\tilde{\mathcal{Q}}^{I}$ generate a smaller $S O(2 \mathcal{N})$ Clifford algebra, whose maximal subalgebra is $S O(3) \times S p(\mathcal{N})$. Therefore states with central charges only build representations of $S p(\mathcal{N})$.

\subsection{CFT realization of extended $D=4$ SUSY}

As it is well known, there is a beautiful relation between the $\mathcal{N}$-extended spacetime supersymmetry algebras and the $n$-extended internal superconformal algebras with corresponding Kac-Moody symmetry $g$. We will assume in the following that we are dealing with holomorphic spacetime supercharges that all originate from the right-moving sector of the compactified string theory, as it is always the case for heterotic string compactifications. As we will discuss, for purely holomorphic supercharges, the massive BPS states with non-vanishing central charges are of perturbative nature. However in type II compactifications, the supercharges can originate from the left-moving as well as the right-moving sector of the string theory. In this case, some of the massive BPS states with central charges are non-perturbative, as they are given in terms of wrapped type II D-branes. These non-perturbative states will not be discussed in this paper.

In SCFT, the holomorphic supercharges $\mathcal{Q}^{I}$ and $\overline{\mathcal{Q}}_{\bar{I}}$ can be always realized by the world-sheet fields of the uncompactified four-dimensional Minkowski spacetime together with those of the internal Kac-Moody symmetries. This fact allows for a completely model-independent realization of the spacetime supersymmetry algebra without any reference to "geometrical" details of the internal SCFT. To be more specific, compactifications to four-dimensional Minkowski spacetime which allow for a CFT description, still have $S O(1,3)$ vectors $i \partial X^{\mu}$ and $\psi^{\mu}$ in their world-sheet theory, the first four components of the ten-dimensional ancestors $i \partial X^{m}$ and $\psi^{m}$. Similarly, the

ten-dimensional $S O(1,9)$ spin field $S_{\alpha}$ factorizes into separate $h=\frac{1}{4}$ and $h=\frac{3}{8}$ primaries $S_{a}$ and $\Sigma$, the former being a Weyl spinor of $S O(1,3)$ and the latter falling into representations of the 
R-symmetry. In fact, both $S O(1,3)$ chiralities can occur, i.e.

$$
S_{\alpha} \equiv S_{a} \Sigma^{I} \oplus S^{\dot{b}} \bar{\Sigma}_{I}
$$

The number of $\left(\Sigma^{I}, \bar{\Sigma}_{I}\right)$ species coincides with the number of spacetime supersymmetries, we will discuss the $\mathcal{N}=4,1,2$ cases below. In each case, the (left- and right-handed) supercharges in their canonical ghost picture are given by

$$
\mathcal{Q}_{a}^{(-1 / 2) I}=\frac{1}{\alpha^{\prime 1 / 4}} \oint \frac{\mathrm{d} z}{2 \pi i} S_{a} \Sigma^{I} \mathrm{e}^{-\phi / 2}, \quad \overline{\mathcal{Q}}_{\bar{J}}^{(-1 / 2), \dot{b}}=\frac{1}{\alpha^{\prime 1 / 4}} \oint \frac{\mathrm{d} z}{2 \pi i} S^{\dot{b}} \bar{\Sigma}_{\bar{J}} \mathrm{e}^{-\phi / 2}
$$

Independent on the fate of the internal spin fields $\Sigma^{I}, \bar{\Sigma}_{I}$, the interactions of the $h=\frac{1}{4}$ spacetime spin fields $S_{a}, S^{\dot{b}}$ with the NS fermions is governed by

$$
\psi^{\mu}(z) S_{a}(w) \sim \frac{\sigma_{a \dot{b}}^{\mu} S^{\dot{b}}(w)}{\sqrt{2}(z-w)^{1 / 2}}+(z-w)^{1 / 2}\left[S_{a}^{\mu}(w)+\frac{1}{\sqrt{2}} \sigma_{a \dot{b}}^{\mu} \partial S^{\dot{b}}(w)\right]+\ldots
$$

In lines with the discussion of subsection 2.3, one can bosonize the left- and right-handed spin fields as $\mathrm{e}^{ \pm i\left(H_{1}+H_{2}\right) / 2}$ and $\mathrm{e}^{ \pm i\left(H_{1}-H_{2}\right) / 2}$, respectively. In order to reconcile bosonization techniques with $S O(1,3)$ symmetry, we align $\mathrm{e}^{ \pm 3 i H_{j} / 2}$ components showing up in the subleading term of the OPE 3.12 into covariant excited spin fields $S_{\mu}^{\dot{b}}, S_{a}^{\mu}$ of weight $h=\frac{5}{4}$ :

$$
S_{a}^{\mu} \leftrightarrow \mathrm{e}^{ \pm 3 i H_{1} / 2} \mathrm{e}^{ \pm i H_{2} / 2}, \quad S_{\mu}^{\dot{b}} \leftrightarrow \mathrm{e}^{ \pm 3 i H_{1} / 2} \mathrm{e}^{\mp i H_{2} / 2}, \quad \bar{\sigma}_{\mu}^{\dot{b} a} S_{a}^{\mu}=\sigma_{a \dot{b}}^{\mu} S_{\mu}^{\dot{b}}=0
$$

A large list of OPEs between $\left(\psi^{\mu}, S_{a}, S^{\dot{b}}, S_{\mu}^{\dot{b}}, S_{a}^{\mu}\right)$ including subleading singularities can be found in appendix B.2.

\subsection{CFT operators in $\mathcal{N}=4$ compactifications}

The internal SCFT in maximally supersymmetric $\mathcal{N}=4$ compactifications to $D=4$ dimensions can be understood in terms of free fields $i \partial Z^{m}, \Psi^{m}$ with $m=4,5, \ldots, 9$ which represent the internal components of the ten-dimensional $i \partial X^{m=0,1, \ldots, 9}, \psi^{m=0,1, \ldots, 9}$ and transform as vectors of the internal rotation group $S O(6)$. The corresponding $h=\frac{3}{8}$ spin fields $\Sigma^{I}$ and $\bar{\Sigma}_{\bar{J}}$, responsible for branch cuts of $\Psi^{m}$, transform as spinors of the $S O(6) \equiv S U(4)$ with left-handed (right-handed) index $I(\bar{J})$. They enter the dimensional reduction $S O(1,9) \rightarrow S O(1,3) \times S O(6)$ of the $D=10$ SUSY charges

$$
\mathcal{Q}_{a}^{(-1 / 2) I}=\frac{1}{\alpha^{\prime 1 / 4}} \oint \frac{\mathrm{d} z}{2 \pi i} S_{a} \Sigma^{I} \mathrm{e}^{-\phi / 2}, \quad \overline{\mathcal{Q}}_{\bar{J}}^{(-1 / 2), \dot{b}}=\frac{1}{\alpha^{\prime 1 / 4}} \oint \frac{\mathrm{d} z}{2 \pi i} S^{\dot{b}} \bar{\Sigma}_{\bar{J}} \mathrm{e}^{-\phi / 2}
$$

where the internal $S O(6) \equiv S U(4)$ is interpreted as the R-symmetry group. The ten-dimensional bosonization prescription can be straightforwardly applied to $\Psi^{m}, \Sigma^{I}, \bar{\Sigma}_{\bar{J}}$ (e.g. $\left.\Sigma^{I} \leftrightarrow \mathrm{e}^{ \pm i\left(H_{3}+H_{4}+H_{5}\right) / 2}\right)$, 
and excited spin fields $\Sigma_{m}^{I}$ and $\bar{\Sigma}_{\bar{J}}^{m}$ of weight $h=\frac{11}{8}$ are constructed in close analogy to their tenand four-dimensional counterparts 2.14 and (3.13):

$$
\Sigma_{m}^{I} \leftrightarrow \quad \mathrm{e}^{ \pm 3 i H_{3} / 2} \mathrm{e}^{ \pm i H_{4} / 2} \mathrm{e}^{ \pm i H_{5} / 2}, \quad \gamma_{\bar{J} I}^{m} \Sigma_{m}^{I}=\bar{\gamma}_{m}^{I \bar{J}} \bar{\Sigma}_{\bar{J}}^{m}=0
$$

The internal supercurrent is built from the $m=4,5, \ldots, 9$ components of its ten-dimensional ancestor (2.6)

$$
G_{\text {int }}=\frac{1}{2 \sqrt{2 \alpha^{\prime}}} i \partial Z_{m} \Psi^{m}
$$

and gives rise to internal central charg $\oint^{7} c=9$. OPEs among the $\Psi^{k}, \Sigma^{I}, \bar{\Sigma}_{\bar{J}}$ and $\Sigma_{m}^{I}, \bar{\Sigma}_{\bar{J}}^{m}$ are gathered in appendix B.3. Identities between six-dimensional gamma and charge conjugation matrices can for instance be found in the appendix of [19]. The following figure 1 aims to give an overview of the conformal fields in the spacetime and $\mathcal{N}=4$ internal $\mathrm{CFT}^{8}$

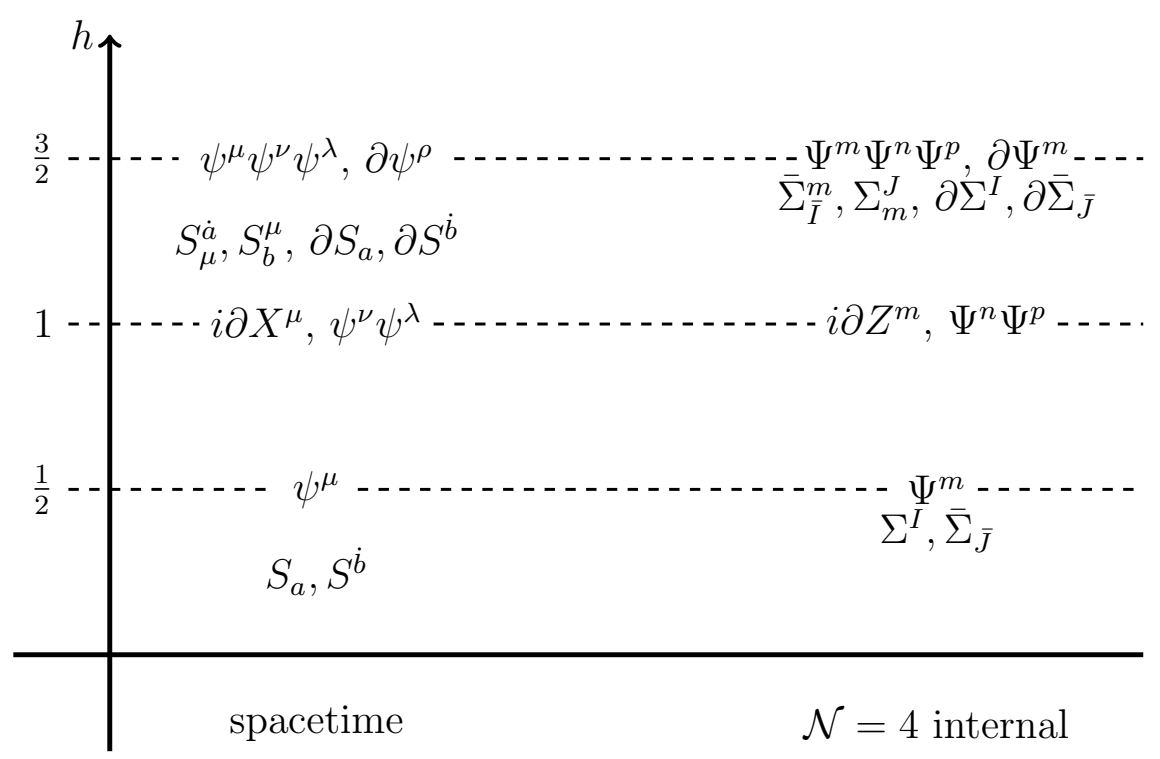

Figure 1: Conformal fields in the spacetime CFT and the internal CFT of $\mathcal{N}=4$ supersymmetric compactifications

${ }^{7}$ The underlying OPEs are

$$
i \partial Z_{m}(z) i \partial Z_{n}(w) \sim \frac{2 \alpha^{\prime} \delta_{m n}^{(6)}}{(z-w)^{2}}+\ldots, \quad \Psi_{m}(z) \Psi_{n}(w) \sim \frac{\delta_{m n}^{(6)}}{z-w}+\ldots .
$$

${ }^{8}$ The fermionic bilinear states $\psi^{\nu} \psi^{\lambda}$ and $\Psi^{n} \Psi^{p}$ at weight $h=1$ by themselves should be eliminated by the GSO projection, but trilinear combinations $\Psi^{m} \psi^{\nu} \psi^{\lambda}$ and $\psi^{\mu} \Psi^{n} \Psi^{p}$ which mix between spacetime components and internal fields would survive after the GSO projection. That is why we include the bilinears into the bookkeeping. 
The higher ghost picture version of the SUSY generators 3.14 is given by

$$
\begin{aligned}
\mathcal{Q}_{a}^{(+1 / 2), I} & =\frac{1}{2 \alpha^{\prime 3 / 4}} \oint \frac{\mathrm{d} z}{2 \pi i}\left[i \partial X_{\mu} \sigma_{a \dot{b}}^{\mu} S^{\dot{b}} \Sigma^{I}+S_{a} i \partial Z^{m} \gamma_{m}^{I \bar{J}} \bar{\Sigma}_{\bar{J}}\right] \mathrm{e}^{+\phi / 2}, \\
\overline{\mathcal{Q}}_{\bar{J}}^{(+1 / 2), \dot{b}} & =\frac{1}{2 \alpha^{\prime 3 / 4}} \oint \frac{\mathrm{d} z}{2 \pi i}\left[i \partial X^{\mu} \sigma_{\mu}^{\dot{b} a} S_{a} \bar{\Sigma}_{\bar{J}}+S^{\dot{b}} i \partial Z_{m} \bar{\gamma}_{\bar{J} I}^{m} \Sigma^{I}\right] \mathrm{e}^{+\phi / 2},
\end{aligned}
$$

their anticommutator with the $(-1 / 2)$ picture analogues 3.14 yields the following ghost-neutral SUSY algebra with nontrivial central charges $\mathcal{Z}^{I J}$ and $\overline{\mathcal{Z}}_{\bar{I} \bar{J}}$ :

$$
\begin{aligned}
& \left\{\mathcal{Q}_{a}^{(+1 / 2), I}, \overline{\mathcal{Q}}_{\bar{J}}^{(-1 / 2), \dot{b}}\right\}=C^{I}{ }_{\bar{J}}\left(\sigma^{\mu} \varepsilon\right)_{a}{ }^{\dot{b}} P_{\mu}, \quad P_{\mu}=\frac{1}{2 \alpha^{\prime}} \oint \frac{\mathrm{d} z}{2 \pi i} i \partial X_{\mu}, \\
& \left\{\mathcal{Q}_{a}^{(+1 / 2), I}, \mathcal{Q}_{b}^{(-1 / 2), J}\right\}=\varepsilon_{a b} \mathcal{Z}^{I J}, \quad \mathcal{Z}^{I J}=\frac{1}{2 \alpha^{\prime}} \oint \frac{\mathrm{d} z}{2 \pi i} i \partial Z^{m}\left(\gamma_{m} C\right)^{I J} \\
& \left\{\overline{\mathcal{Q}}_{\bar{I}}^{(+1 / 2), \dot{a}}, \overline{\mathcal{Q}}_{\bar{J}}^{(-1 / 2), \dot{b}}\right\}=\varepsilon^{\dot{a} \dot{b}} \overline{\mathcal{Z}}_{\bar{I} \bar{J}}, \quad \overline{\mathcal{Z}}_{\bar{I} \bar{J}}=\frac{1}{2 \alpha^{\prime}} \oint \frac{\mathrm{d} z}{2 \pi i} i \partial Z_{m}\left(\bar{\gamma}^{m} C\right)_{\bar{I} \bar{J}} .
\end{aligned}
$$

The central charges arise due to poles in the operator product expansion of $\mathcal{Q}_{a}^{(+1 / 2), I}$ and $\mathcal{Q}_{b}^{(-1 / 2), J}$ caused by internal free fermions and bosons $\Psi^{m}$ and $\partial Z_{m}$. The latter appear in the internal supercurrent $G_{\mathrm{int}} \sim i \partial Z_{m} \Psi^{m}$ and generate an internal Kac-Moody algebra

$$
g=S O(6) \times[U(1)]^{6}
$$

with dimension one currents

$$
j_{S O(6)}^{m n}(z)=\Psi^{m} \Psi^{n}(z), \quad j_{U(1)^{6}}^{m}(z)=i \partial Z^{m}(z)
$$

The fields $Z_{m}(z)$ can be viewed as the coordinates of a (holomorphic) torus compactification on a six-dimensional torus $T^{6}$. Their world-sheet superpartners $\Psi^{m}$ generate a $U(1)^{6}$ spacetime gauge symmetry, and the six spacetime gauge bosons are the six graviphotons, which arise in any compactification on a (holomorphic) six-torus. States that carry non-vanishing internal momenta $p^{m}$ on the (holomorphic) six-torus always have the following field as part of their vertex operator:

$$
\left|p^{m}\right\rangle \sim \mathrm{e}^{i p^{m} Z_{m}(z)}
$$

Switching to the more convenient bispinor basis, the six central charge operators (in the zero ghost picture) of the $\mathcal{N}=4$ supersymmetry algebra are nothing else than the free bosons $Z^{m}$ :

$$
\mathcal{Z}^{I J}(z)=\frac{1}{2 \alpha^{\prime}}\left(\gamma_{m} C\right)^{I J} i \partial Z^{m}(z)
$$

It follows that the internal momentum states $\left|p^{m}\right\rangle$ are precisely those states that carry nonvanishing $\mathcal{N}=4$ central charges. They break the internal world-sheet $S O(6)$ symmetry to $S O(5)$. 
At the same time, states with non-vanishing momenta $p^{m}$ build representations of the spacetime automorphism group for massive states with central charges, which is $S p(4) \cong S O(5)$. On the other hand, states with vanishing internal momenta, $\left|p^{m}=0\right\rangle$, build internal $S O(6)$ representations, respectively at the same time representations of the group $U S p(8)$, which classifies massive states without central charges. The subsequent discussions only take into account the states at zero internal momentum $\left(p^{m}=0\right)$.

\subsection{CFT operators in $\mathcal{N}=1$ compactifications}

In this subsection, we summarize universal aspects of internal $c=9$ SCFTs describing $D=4$ superstring compactifications which preserve $\mathcal{N}=1$ SUSY in spacetime [12, 13, 14]. The existence of one supercharge species

$$
\mathcal{Q}_{a}^{(-1 / 2)}=\frac{1}{\alpha^{1 / 4}} \oint \frac{\mathrm{d} z}{2 \pi i} S_{a} \Sigma^{+} \mathrm{e}^{-\phi / 2}, \quad \overline{\mathcal{Q}}^{(-1 / 2) \dot{b}}=\frac{1}{\alpha^{\prime 1 / 4}} \oint \frac{\mathrm{d} z}{2 \pi i} S^{\dot{b}} \Sigma^{-} \mathrm{e}^{-\phi / 2}
$$

with $h=\frac{3}{8}$ spin fields $\Sigma^{ \pm}$implies that the world-sheet supersymmetry is enhanced to $\mathcal{N}=2$. This can be traced back to the existence of a $U(1)$ Kac-Moody current $\mathcal{J}$ of $h=1$ which emerges from the mutual OPEs of spin fields with opposite charge:

$$
\Sigma^{ \pm}(z) \Sigma^{\mp}(w) \sim \frac{1}{(z-w)^{3 / 4}} \pm \frac{\sqrt{3}}{2}(z-w)^{1 / 4} \mathcal{J}(w)+\ldots
$$

The internal supercurrents $G_{\text {int }}^{ \pm}$can be split into two components of opposite $U(1)$ charge,

$$
G_{\text {int }}=\frac{1}{\sqrt{2}}\left(G_{\text {int }}^{+}+G_{\text {int }}^{-}\right)
$$

subject to the superconformal $\mathcal{N}=2$ algebra ${ }^{9}$

$$
\begin{aligned}
\mathcal{J}(z) \mathcal{J}(w) & \sim \frac{1}{(z-w)^{2}}+\mathcal{J}(w) \mathcal{J}(w)+\ldots \\
\mathcal{J}(z) G_{\text {int }}^{ \pm}(w) & \sim \pm \frac{G_{\text {int }}^{ \pm}(w)}{\sqrt{3}(z-w)}+\mathcal{J}(w) G_{\text {int }}^{ \pm}(w)+\ldots \\
G_{\text {int }}^{ \pm}(z) G_{\text {int }}^{ \pm}(w) & \sim G_{\text {int }}^{ \pm}(w) G_{\text {int }}^{ \pm}(w)+\ldots \\
G_{\text {int }}^{ \pm}(z) G_{\text {int }}^{\mp}(w) & \sim \frac{3 / 2}{(z-w)^{3}} \pm \frac{\sqrt{3} \mathcal{J}(w)}{2(z-w)^{2}}+\frac{2 T_{\text {int }}(w) \pm \sqrt{3} \partial \mathcal{J}(w)}{4(z-w)}+\ldots
\end{aligned}
$$

\footnotetext{
${ }^{9}$ In contrast to [12, 13, 14, we normalize $\mathcal{J}$ such that it has canonical two-point functions $\langle\mathcal{J}(z) \mathcal{J}(w)\rangle=$ $1 \cdot(z-w)^{-2}$. This simplifies (subleading) OPE coefficients and normalization factors in vertex operators.
} 
with internal $c=9$ energy momentum tensor $T_{\text {int }}$. The OPE of alike spin fields gives rise to new $h=\frac{3}{2}$ Virasoro primary operators

$$
\Sigma^{ \pm}(z) \Sigma^{ \pm}(w) \sim(z-w)^{3 / 4} \mathcal{O}^{ \pm}(w)+\ldots
$$

with twice the $U(1)$ charge of the spin fields, and iterated OPEs with $\Sigma^{ \pm}$create an infinite tower of further conformal primaries with higher weights and charges.

A large sector of the internal CFT can be captured by bosonization. Let $H(z)$ denote a canonically normalized free \& chiral boson, then we have the following representation for some for the aforementioned operators:

$$
\mathcal{J} \equiv i \partial H, \quad \Sigma^{ \pm} \equiv \mathrm{e}^{ \pm i \sqrt{3} H / 2}, \quad \mathcal{O}^{ \pm} \equiv \mathrm{e}^{ \pm i \sqrt{3} H}
$$

However, the internal supercurrent (or energy momentum tensor) cannot be fully bosonized. Instead, we can represent $G_{\text {int }}^{ \pm}$as

$$
G_{\text {int }}^{ \pm}=\sqrt{\frac{3}{2}} \mathrm{e}^{ \pm \frac{i}{\sqrt{3}} H} g^{ \pm}
$$

where the $h=\frac{4}{3}$ operators $g^{ \pm}$are local with respect to $H$ and satisfy

$$
\begin{aligned}
g^{ \pm}(z) g^{\mp}(w) & \sim \frac{1}{(z-w)^{8 / 3}}+\frac{0}{(z-w)^{5 / 3}}+\ldots \\
g^{ \pm}(z) g^{ \pm}(w) & \sim \frac{g^{ \pm}(w) g^{ \pm}(w)}{(z-w)^{1 / 3}}+\ldots
\end{aligned}
$$

On these grounds, we can understand the OPE of the supercurrent with internal spin fields,

$$
\begin{aligned}
G_{\text {int }}^{ \pm}(z) \Sigma^{\mp}(w) & \sim \sqrt{\frac{3}{2}} \frac{\tilde{\Sigma}^{\mp}(w)}{(z-w)^{1 / 2}}+\ldots \\
G_{\text {int }}^{ \pm}(z) \Sigma^{ \pm}(w) & \sim(z-w)^{1 / 2} g^{ \pm} \mathrm{e}^{ \pm \frac{5 i}{2 \sqrt{3}}}(w)+\ldots
\end{aligned}
$$

which introduces excited spin fields $\tilde{\Sigma}^{ \pm}$of $h=\frac{11}{8}$ in case of opposite $U(1)$ charges $G_{\text {int }}^{ \pm} \leftrightarrow \Sigma^{\mp}$,

$$
\tilde{\Sigma}^{ \pm} \equiv g^{\mp} \mathrm{e}^{ \pm \frac{i}{2 \sqrt{3}} H}
$$

Figure 2 gives an overview of the universal Virasoro primaries in the internal $c=9$ SCFT. More detailed OPEs including subleading singularities can be found in appendix B.4.

From these OPEs, we obtain the following $+1 / 2$ ghost picture version for the SUSY charge

$$
\mathcal{Q}_{a}^{(+1 / 2)}=\oint \frac{\mathrm{d} z}{2 \pi i}\left[\frac{\sqrt{3}}{\alpha^{\prime 1 / 4}} S_{a} \tilde{\Sigma}^{+}+\frac{1}{2 \alpha^{\prime 3 / 4}} i \partial X_{\mu} \sigma_{a \dot{b}}^{\mu} S^{\dot{b}} \Sigma^{+}\right] \mathrm{e}^{+\phi / 2}
$$




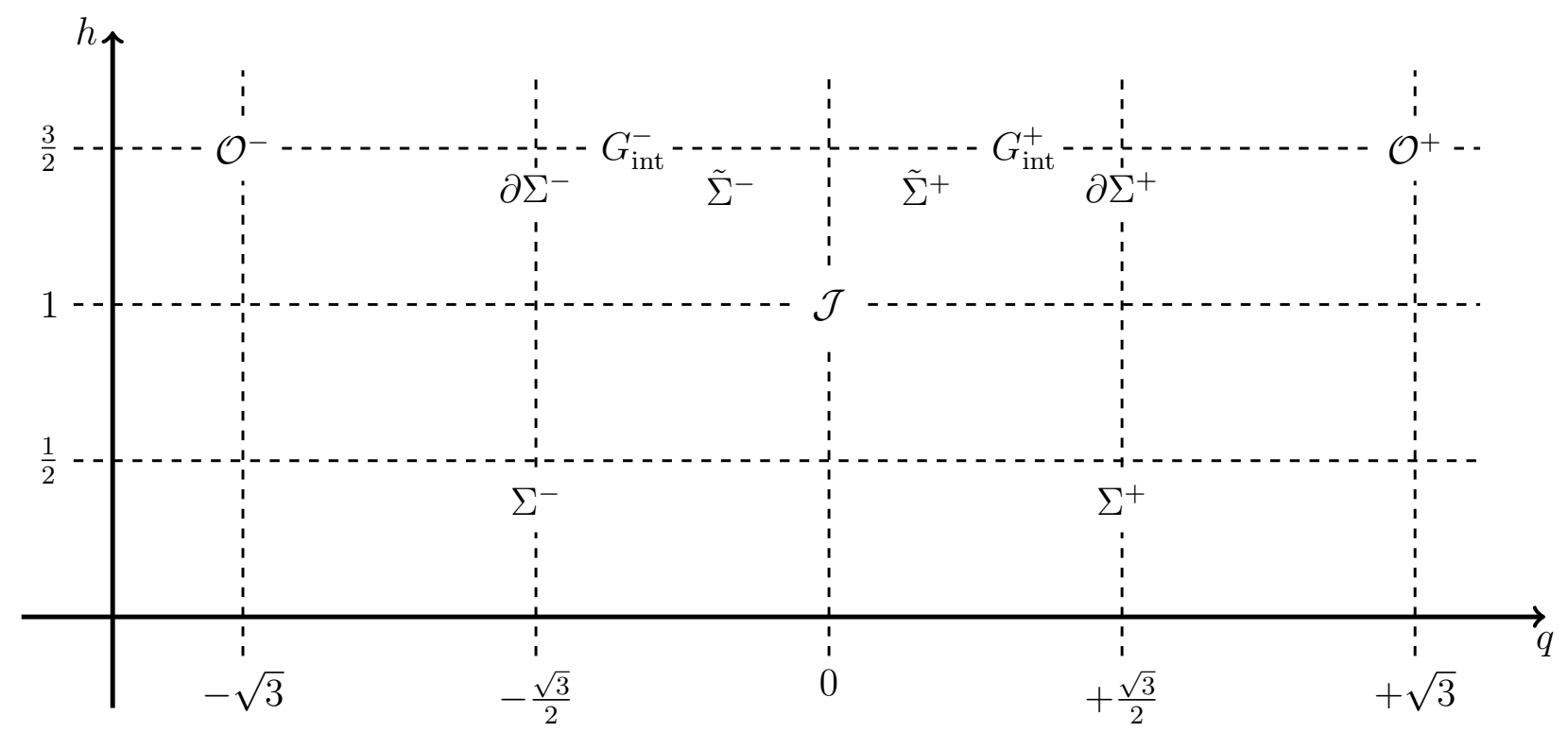

Figure 2: Conformal fields in the $\mathcal{N}=1$ internal CFT, together with their weight $h$ and $U(1)$ charge $q$.

$$
\overline{\mathcal{Q}}^{(+1 / 2) \dot{b}}=\oint \frac{\mathrm{d} z}{2 \pi i}\left[\frac{\sqrt{3}}{\alpha^{\prime 1 / 4}} S^{\dot{b}} \tilde{\Sigma}^{-}+\frac{1}{2 \alpha^{\prime 3 / 4}} i \partial X^{\mu} \bar{\sigma}_{\mu}^{\dot{b} a} S_{a} \Sigma^{-}\right] \mathrm{e}^{+\phi / 2}
$$

which yield the $\mathcal{N}=1$ SUSY algebra

$$
\left\{\mathcal{Q}_{a}^{(+1 / 2)}, \overline{\mathcal{Q}}^{(-1 / 2), \dot{b}}\right\}=\left(\sigma^{\mu} \varepsilon\right)_{a}^{\dot{b}} P_{\mu}, \quad P_{\mu}=\frac{1}{2 \alpha^{\prime}} \oint \frac{\mathrm{d} z}{2 \pi i} i \partial X_{\mu}
$$

\subsection{CFT operators in $\mathcal{N}=2$ compactifications}

In superstring compactifications which preserve $\mathcal{N}=2$ spacetime SUSY, it can be shown along the lines of [13, 14] that the internal CFT splits into two decoupled sectors with central charges $c=6$ and $c=3$, respectively. Starting point are the two supercharges

$$
\mathcal{Q}_{a}^{(-1 / 2), i}=\frac{1}{\alpha^{\prime 1 / 4}} \oint \frac{\mathrm{d} z}{2 \pi i} S_{a} \Sigma^{i} \mathrm{e}^{-\phi / 2}, \quad \overline{\mathcal{Q}}^{(-1 / 2), \dot{b} i}=\frac{1}{\alpha^{\prime 1 / 4}} \oint \frac{\mathrm{d} z}{2 \pi i} S^{\dot{b}} \bar{\Sigma}^{i} \mathrm{e}^{-\phi / 2}
$$

containing two species of spin fields $\Sigma^{i=1,2}$ and $\bar{\Sigma}^{i=1,2}$. The latter turn out to factorize into decoupled primaries $\lambda^{i}$ and $\mathrm{e}^{ \pm i H / 2}$ from the $c=6$ and $c=3$ sector, respectively:

$$
\Sigma^{i}=\lambda^{i} \mathrm{e}^{+i H / 2}, \quad \bar{\Sigma}^{i}=\lambda^{i} \mathrm{e}^{-i H / 2} .
$$

The $c=3$ part can be represented in terms of a single free chiral boson $H$ subject to (2.12). Its contribution $\frac{1}{2}(i \partial H)^{2}$ to the $c=3$ energy momentum tensor assigns conformal weight $h\left(\mathrm{e}^{ \pm i H / 2}\right)=$ 
$1 / 8$ (or more generally, $\left.h\left(\mathrm{e}^{i q H}\right)=q^{2} / 2\right)$. Moreover, OPEs of the partial spin fields $\mathrm{e}^{ \pm i H / 2}$ introduce $h=\frac{1}{2}$ fermions $\mathrm{e}^{ \pm i H}$ and excited spin fields $\mathrm{e}^{ \pm 3 i H / 2}$ of weight $h=\frac{9}{8}$.

On the other hand, the $\lambda^{i}$ fields from the $c=6$ sector have weight $h\left(\lambda^{i}\right)=1 / 4$ and form an $S U(2)$ doublet. Their operator algebra ${ }^{10}$ gives rise to an $S U(2)$ triplet of $h=1$ currents $\mathcal{J}^{A=1,2,3}$ :

$$
\lambda^{i}(z) \lambda^{j}(w) \sim \frac{\varepsilon^{i j}}{(z-w)^{1 / 2}}+\frac{1}{\sqrt{2}}(z-w)^{1 / 2}\left(\tau_{A} \varepsilon\right)^{i j} \mathcal{J}^{A}(w)+\ldots
$$

The $\tau_{A}$ denote the standard (traceless) $S U(2)$ Pauli matrices $\left\{\left(\begin{array}{ll}0 & 1 \\ 1 & 0\end{array}\right),\left(\begin{array}{cc}0 & -i \\ i & 0\end{array}\right),\left(\begin{array}{ccc}1 & 0 \\ 0 & -1\end{array}\right)\right\}$ subject to the multiplication rule $\tau_{A} \tau_{B}=\delta_{A B}+i \varepsilon_{A B C} \tau^{C}$.

The currents obey the $S U(2)$ current algebra at level $k=1$, we use normalization conventions

$$
\mathcal{J}^{A}(z) \mathcal{J}^{B}(w) \sim \frac{\delta^{A B}}{(z-w)^{2}}+\frac{i \sqrt{2} \varepsilon^{A B C} \mathcal{J}_{C}(w)}{z-w}+\ldots
$$

in which their interaction with the spin fields is governed by

$$
\begin{aligned}
\mathcal{J}^{A}(z) \lambda^{i}(w) & \sim \frac{\left(\tau^{A}\right)^{i}{ }_{j} \lambda^{j}(w)}{\sqrt{2}(z-w)}+\sqrt{2}\left(\tau^{A}\right)^{i}{ }_{j} \partial \lambda^{j}(w)+\ldots \\
\lambda^{i}(z) \mathcal{J}^{A}(w) & \sim \frac{\left(\tau^{A}\right)^{i}{ }_{j} \lambda^{j}(w)}{\sqrt{2}(z-w)}-\frac{1}{\sqrt{2}}\left(\tau^{A}\right)^{i}{ }_{j} \partial \lambda^{j}(w)+\ldots
\end{aligned}
$$

Note that also the $\lambda^{i}$ and $\mathcal{J}^{A}$ fit into a bosonization scheme according to

$$
\mathcal{J}^{A=3} \equiv i \partial H_{3}, \quad \mathcal{J}^{A=1} \pm i \mathcal{J}^{A=2} \equiv \sqrt{2} \mathrm{e}^{ \pm i \sqrt{2} H_{3}}, \quad \lambda^{i=1,2}=\mathrm{e}^{ \pm i H_{3} / \sqrt{2}}
$$

with $H_{3}$ being nonsingular with respect to the $c=3$ boson $H$. This fixes the choice of the $S U(2)$ Cartan subalgebra.

The world-sheet supercurrents associated with the two decoupled CFTs,

$$
G_{\text {int }} \equiv G_{c=3}+G_{c=6}
$$

can be split according to their charges under the $h=1$ currents. In the $c=3$ sector, we find a

\footnotetext{
${ }^{10}$ The contraction rules for the antisymmetric $\varepsilon^{i j}, \varepsilon_{i j}$ tensors introduce signs in some of the OPEs:
}

$$
\lambda_{i}(z) \lambda^{j}(w) \sim \frac{+\delta_{i}^{j}}{(z-w)^{1 / 2}}, \quad \lambda_{i}(z) \lambda_{j}(w) \sim \frac{-\varepsilon_{i j}}{(z-w)^{1 / 2}}, \quad \lambda^{i}(z) \lambda_{j}(w) \sim \frac{-\delta_{j}^{i}}{(z-w)^{1 / 2}} .
$$


free field representation in terms of internal $h=1$ coordinate: ${ }^{11} i \partial Z^{ \pm}$,

$$
G_{c=3}=\frac{1}{2 \sqrt{2 \alpha^{\prime}}}\left(i \partial Z^{+} \mathrm{e}^{-i H}+i \partial Z^{-} \mathrm{e}^{i H}\right)
$$

The fermions $\Psi^{ \pm}(z)=e^{ \pm i H(z)}$ together with the free bosons $Z^{ \pm}$generate an internal Kac-Moody algebra

$$
g=S O(2) \times[U(1)]^{2}
$$

with dimension one currents

$$
j_{S O(2)}(z)=\Psi^{+} \Psi^{-}(z)=i \partial H(z), \quad j_{U(1)}^{ \pm}(z)=i \partial Z^{ \pm}(z)
$$

As for the $\mathcal{N}=4$ case, the fields $Z_{ \pm}(z)$ can be viewed as the coordinates of a (holomorphic) torus compactification on a two-dimensional torus $T^{2}$.

Also the supercurrent of the $c=6$ sector cannot be fully built from the bosonization prescription (3.52, it additionally requires the introduction of an $S U(2)$ doublet of $h=5 / 4$ fields $g_{i}$ :

$$
G_{c=6}=\frac{1}{\sqrt{2}}\left(\mathrm{e}^{i H_{3} / \sqrt{2}} g_{1}+\mathrm{e}^{-i H_{3} / \sqrt{2}} g_{2}\right)=\frac{1}{\sqrt{2}} \lambda^{i} g_{i}
$$

The $g_{i}$ decouple from the $\lambda^{i}$ and $\mathcal{J}^{A}$, and their $\mathrm{OPE}$

$$
g_{i}(z) g_{j}(w) \sim \frac{\varepsilon_{i j}}{(z-w)^{5 / 2}}+\frac{0}{(z-w)^{3 / 2}}+\ldots
$$

makes sure that the supercurrents satisfy the required $\mathcal{N}=4$ superconformal algebra at $c=6$. A summary of operators in the internal SCFTs common to $\mathcal{N}=2$ compactifications are presented in figure 3 .

The internal supercurrent yields the following higher ghost picture SUSY charges:

$$
\mathcal{Q}_{a}^{(+1 / 2), i}=\frac{1}{\sqrt{2} \alpha^{\prime 3 / 4}} \oint \frac{\mathrm{d} z}{2 \pi i}\left[\frac{1}{\sqrt{2}} i \partial X_{\mu} \sigma_{a \dot{b}}^{\mu} S^{\dot{b}} \lambda^{i} \mathrm{e}^{i H / 2}+i \partial Z^{+} S_{a} \lambda^{i} \mathrm{e}^{-i H / 2}\right.
$$

${ }^{11}$ As usual, the OPEs between $i \partial Z^{ \pm}$are normalized as

$$
i \partial Z^{ \pm}(z) i \partial Z^{\mp}(w) \sim \frac{2 \alpha^{\prime}}{(z-w)^{2}}+\ldots, \quad i \partial Z^{ \pm}(z) i \partial Z^{ \pm}(w) \sim i \partial Z^{ \pm}(w) i \partial Z^{ \pm}(w)+\ldots .
$$

${ }^{12} \varepsilon$ contractions yield signs opposite to the $\lambda^{i} \lambda_{j}$ case:

$$
g^{i}(z) g_{j}(w) \sim \frac{+\delta_{j}^{i}}{(z-w)^{5 / 2}}, \quad g^{i}(z) g^{j}(w) \sim \frac{-\varepsilon^{i j}}{(z-w)^{5 / 2}}, \quad g_{i}(z) g^{j}(w) \sim \frac{-\delta_{i}^{j}}{(z-w)^{5 / 2}} .
$$




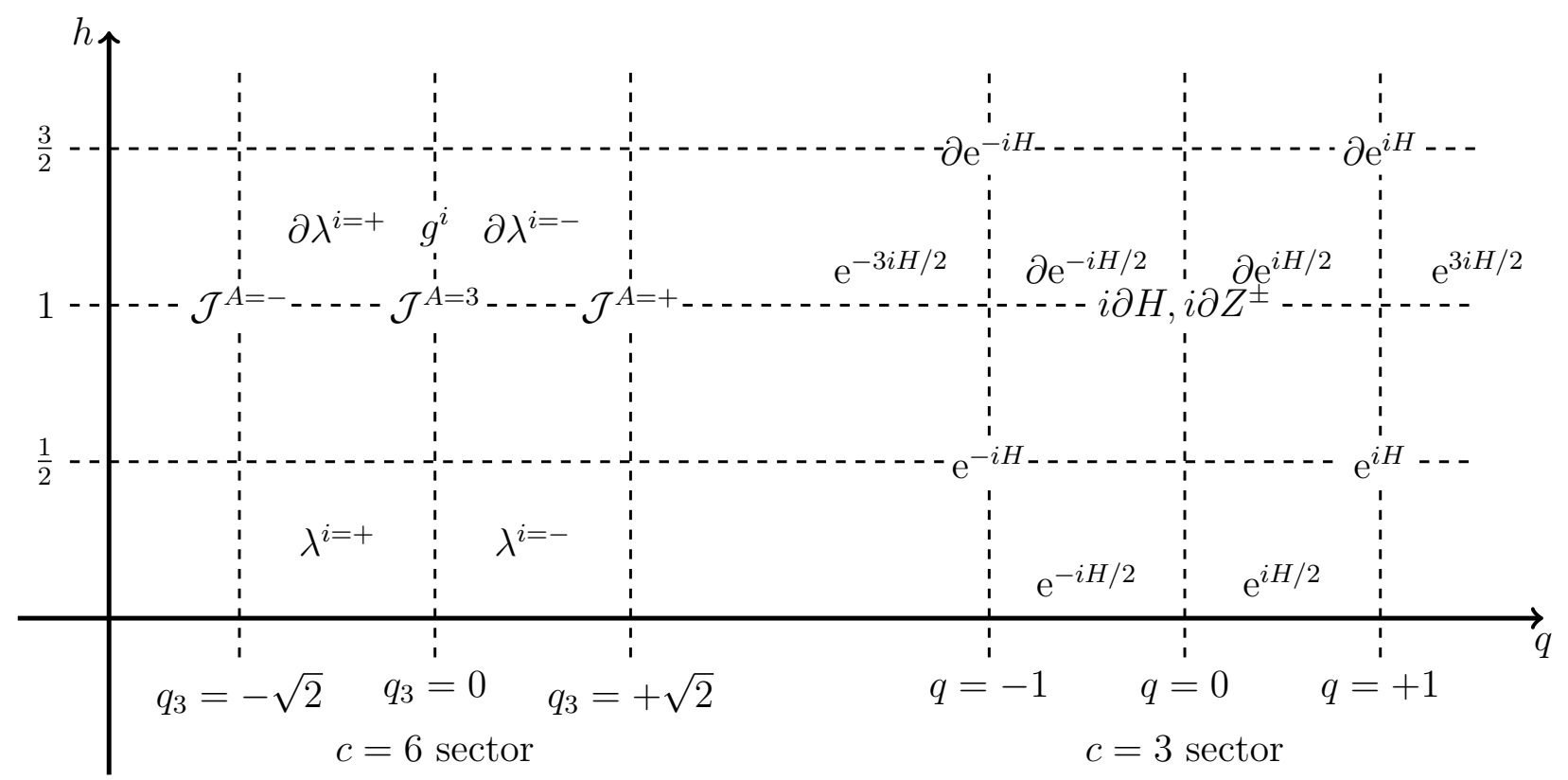

Figure 3: Universal operator content of the internal CFT associated with $\mathcal{N}=2$ spacetime SUSY, including weight $h$ and charges $q_{3}, q$ under $i \partial H_{3}$ and $i \partial H$, respectively.

$$
\begin{aligned}
& \left.-2 \sqrt{\alpha^{\prime}} g^{i} S_{a} \mathrm{e}^{i H / 2}\right] \mathrm{e}^{\phi / 2} \\
& \overline{\mathcal{Q}}^{(+1 / 2), \dot{b} i}=\frac{1}{\sqrt{2} \alpha^{\prime 3 / 4}} \oint \frac{\mathrm{d} z}{2 \pi i}\left[\frac{1}{\sqrt{2}} i \partial X^{\mu} \bar{\sigma}_{\mu}^{\dot{b} a} S_{a} \lambda^{i} \mathrm{e}^{-i H / 2}+i \partial Z^{-} S^{\dot{b}} \lambda^{i} \mathrm{e}^{i H / 2}\right. \\
& \left.-2 \sqrt{\alpha^{\prime}} g^{i} S^{\dot{b}} \mathrm{e}^{-i H / 2}\right] \mathrm{e}^{\phi / 2}
\end{aligned}
$$

The anticommutator of equal chirality generators gives rise to a complex central charge operator, which can be written in terms of the free bosons $Z^{ \pm}$:

$$
\begin{aligned}
\left\{\mathcal{Q}_{a}^{(+1 / 2), i}, \mathcal{Q}_{b}^{(-1 / 2), j}\right\} & =\varepsilon_{a b} \mathcal{Z}^{i j}, & \mathcal{Z}^{i j} & =\frac{\varepsilon^{i j}}{\sqrt{2} \alpha^{\prime}} \oint \frac{\mathrm{d} z}{2 \pi i} i \partial Z^{+}, \\
\left\{\overline{\mathcal{Q}}^{(+1 / 2), \dot{a} i}, \overline{\mathcal{Q}}^{(-1 / 2), \dot{b} j}\right\} & =\varepsilon^{\dot{a} \dot{b}} \overline{\mathcal{Z}}^{i j}, & \overline{\mathcal{Z}}^{i j} & =\frac{\varepsilon^{i j}}{\sqrt{2} \alpha^{\prime}} \oint \frac{\mathrm{d} z}{2 \pi i} i \partial Z^{-} .
\end{aligned}
$$

It again follows that the internal momentum states $\left|p^{ \pm}\right\rangle$of the two-torus are precisely those states that carry non-vanishing $\mathcal{N}=2$ central charges. They completely break the internal world-sheet $S O(2)$ symmetry. On the other hand, states with vanishing internal momenta, $\left|p^{ \pm}=0\right\rangle$, build internal $S O(2)$ representations, resp. representations of the group $U S p(4)$, which classifies the $\mathcal{N}=2$ massive states without central charges. 


\subsection{Summary of CFT operators}

To conclude this section on the internal SCFTs associated with $D=4$ compactifications of different supercharges, figure 4 summarizes the field content of the different sectors. This is a good reference to build the most general ansatz for physical vertex operators.

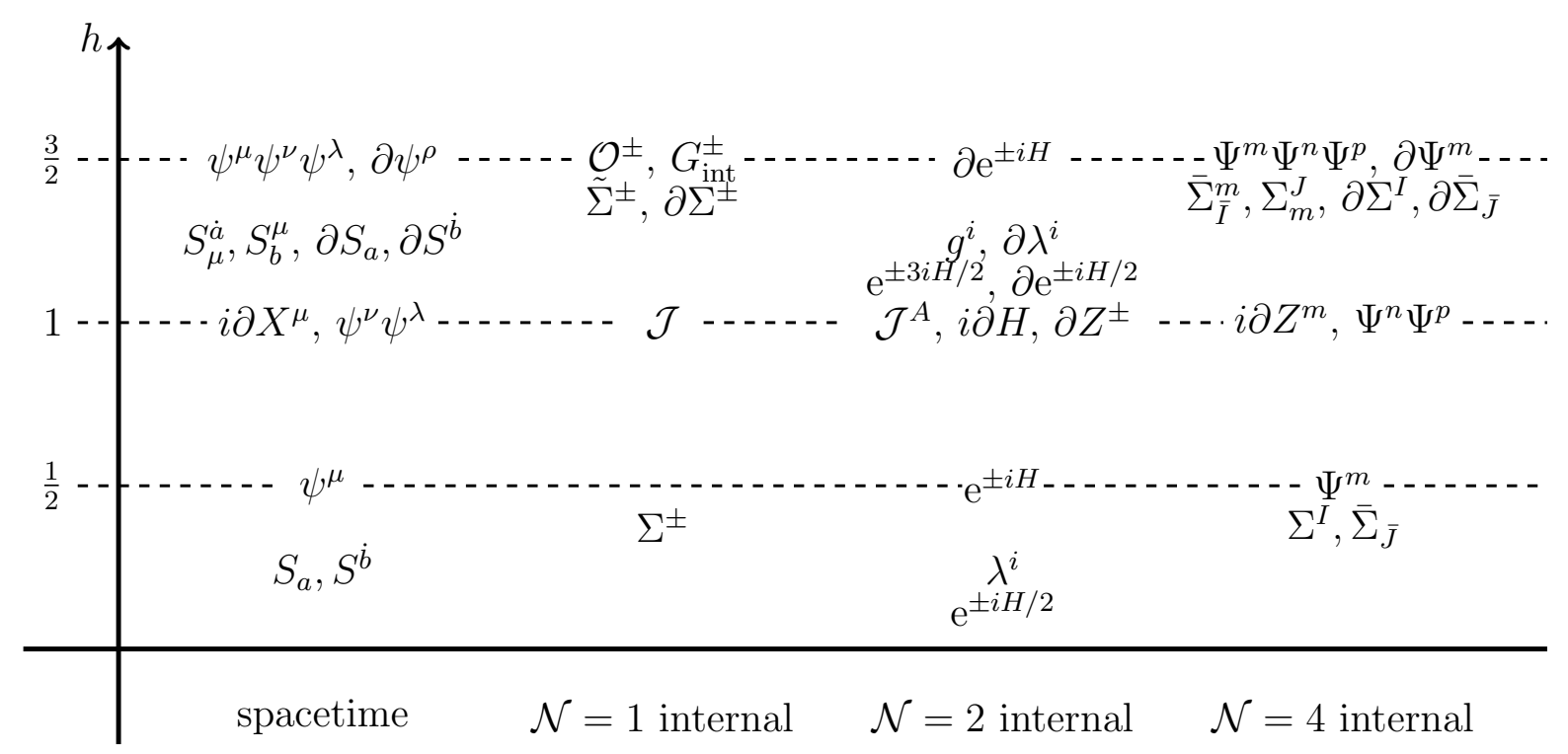

Figure 4: Conformal fields together with their weight in various decoupling CFT sectors

\section{Massive supermultiplets for $\mathcal{N}=4$ SUSY}

Having introduced the CFT setup for the construction of massive string state, let us now turn to explicit vertex operators on the first mass level. We will first of all examine the four-dimensional field content of maximally supersymmetric superstring compactifications to $D=4$ with $\mathcal{N}=4$ SUSY. This is the dimensional reduction of the ten-dimensional multiplet, so we will again find all the 256 states which have been discussed from the $D=10$ viewpoint in section 2. They form a massive $\mathcal{N}=4$ multiplet in four dimensions for which we will work out the spin and R-symmetry content as well as the SUSY transformations. 


\subsection{NS sector}

With the internal CFT operators from figure 1 at hand, the following $h=3 / 2$ combinations must be considered in the most general NS vertex operator at first mass level:

$$
\begin{aligned}
V^{(-1)}= & \left(\alpha_{\mu \nu} i \partial X^{\mu} \psi^{\nu}+e_{\mu \nu \lambda} \psi^{\mu} \psi^{\nu} \psi^{\lambda}+h_{\mu} \partial \psi^{\mu}+\beta_{\mu}^{m} i \partial X^{\mu} \Psi_{m}\right. \\
& +\gamma_{\mu}^{m} \psi^{\mu} i \partial Z_{m}+d_{\mu}^{m n} \psi^{\mu} \Psi_{m} \Psi_{n}+Y^{m} \partial \Psi_{m}+\omega_{\mu \nu}^{m} \psi^{\mu} \psi^{\nu} \Psi_{m} \\
& \left.+\zeta^{m n} i \partial Z_{m} \Psi_{n}+\Omega^{m n p} \Psi_{m} \Psi_{n} \Psi_{p}\right) \mathrm{e}^{-\phi} \mathrm{e}^{i k \cdot X}
\end{aligned}
$$

Requiring vanishing $Q_{1}$ variation for 4.1) implies the following on-shell constraints for the ten wavefunctions above:

$$
\begin{array}{rlrl}
0 & =\alpha_{\mu}^{\mu}+k^{\mu} h_{\mu}+\zeta_{m}^{m} & 0 & =2 \alpha^{\prime} Y^{m}+k^{\mu} \gamma_{\mu}^{m} \\
0 & =\alpha_{[\mu \nu]}+3 k^{\lambda} e_{\lambda \mu \nu} & 0 & =\beta_{\mu}^{m}-\gamma_{\mu}^{m}+2 k^{\lambda} \omega_{\lambda \mu}^{m} \\
0 & =2 \alpha^{\prime} \alpha_{\mu \nu} k^{\nu}+h_{\mu} & 0 & =k^{\mu} d_{\mu}^{m n}+\zeta^{[m n]}
\end{array}
$$

This leaves the following 128 physical solutions

- one transverse and traceless spin two tensor

$$
V_{\alpha}^{(-1)}=\frac{1}{\sqrt{2 \alpha^{\prime}}} \alpha_{\mu \nu} i \partial X^{\mu} \psi^{\nu} \mathrm{e}^{-\phi} \mathrm{e}^{i k \cdot X}, \quad k^{\mu} \alpha_{\mu \nu}=\alpha_{[\mu \nu]}=\alpha_{\mu}{ }^{\mu}=0
$$

- 27 transverse vectors (in the vector and two-form representations of the R-symmetry $S O(6)$ )

$$
\begin{aligned}
V_{d}^{(-1)}= & \frac{1}{2} d_{\mu}^{m n} \psi^{\mu} \Psi_{m} \Psi_{n} \mathrm{e}^{-\phi} \mathrm{e}^{i k \cdot X}, \quad k^{\mu} d_{\mu}^{m n}=0 \\
V_{\beta^{ \pm}}^{(-1)}= & \frac{1}{2 \sqrt{2 \alpha^{\prime}}} \beta_{\mu}^{ \pm, m}\left(i \partial X^{\mu} \Psi_{m}+i \partial Z_{m} \psi^{\mu}\right. \\
& \left. \pm i \alpha^{\prime} \varepsilon^{\mu \nu \lambda \rho} k_{\nu} \psi_{\lambda} \psi_{\rho} \Psi_{m}\right) \mathrm{e}^{-\phi} \mathrm{e}^{i k \cdot X}, \quad k^{\mu} \beta_{\mu}^{ \pm, m}=0
\end{aligned}
$$

- 42 scalar degrees of freedom (scalars, spin two and and three-form with respect to $S O(6)$ )

$$
\begin{aligned}
V_{\Phi^{ \pm}}^{(-1)} & =\frac{1}{2 \sqrt{2 \alpha^{\prime}}} \Phi^{ \pm}\left[\left(\eta_{\mu \nu}+2 \alpha^{\prime} k_{\mu} k_{\nu}\right) i \partial X^{\mu} \psi^{\nu}+2 \alpha^{\prime} k_{\mu} \partial \psi^{\mu}\right. \\
& \left. \pm \frac{i \alpha^{\prime}}{3} \varepsilon_{\mu \nu \lambda \rho} \psi^{\mu} \psi^{\nu} \psi^{\lambda} k^{\rho}\right] \mathrm{e}^{-\phi} \mathrm{e}^{i k \cdot X} \\
V_{\zeta}^{(-1)} & =\frac{1}{\sqrt{2 \alpha^{\prime}}} \zeta^{m n} i \partial Z_{m} \Psi_{n} \mathrm{e}^{-\phi} \mathrm{e}^{i k \cdot X}, \quad \zeta^{[m n]}=\zeta^{m}=0 \\
V_{\Omega}^{(-1)} & =\Omega^{m n p} \Psi_{m} \Psi_{n} \Psi_{p} \mathrm{e}^{-\phi} \mathrm{e}^{i k \cdot X} .
\end{aligned}
$$


The 46 spurious NS sector states from ten dimensions are aligned into six representations of $S O(1,3) \times S O(6)$. They can be constructively obtained as BRST variations of ghost charge -2 objects, see 2.10):

$$
\begin{aligned}
V_{\pi(\mathrm{sp})}^{(-1)} & \sim\left[\left(\pi_{\mu} k_{\nu}+k_{\mu} \pi_{\nu}\right) i \partial X^{\mu} \psi^{\nu}+2 \pi_{\mu} \partial \psi^{\mu}\right] \mathrm{e}^{-\phi} \mathrm{e}^{i k \cdot X}, \quad k^{\mu} \pi_{\mu}=0 \\
V_{\Sigma(\mathrm{sp})}^{(-1)} & \sim\left[2 \Sigma_{[\mu \nu]} i \partial X^{\mu} \psi^{\nu}+2 \alpha^{\prime} \Sigma_{[\mu \nu} k_{\lambda]} \psi^{\mu} \psi^{\nu} \psi^{\lambda}\right] \mathrm{e}^{-\phi} \mathrm{e}^{i k \cdot X}, \quad k^{\mu} \Sigma_{\mu \nu}=0 \\
V_{\Lambda_{1}(\mathrm{sp})}^{(-1)} & \sim \Lambda_{1}\left[\left(\eta_{\mu \nu}+4 \alpha^{\prime} k_{\mu} k_{\nu}\right) i \partial X^{\mu} \psi^{\nu}+6 \alpha^{\prime} k_{\mu} \partial \psi^{\mu}+i \partial Z_{m} \Psi^{m}\right] \mathrm{e}^{-\phi} \mathrm{e}^{i k \cdot X} \\
V_{\Lambda_{2}(\mathrm{sp})}^{(-1)} & \sim \Lambda_{2}^{m}\left(k_{\mu}\left[i \partial X^{\mu} \Psi_{m}+i \partial Z_{m} \psi^{\mu}\right]+2 \partial \Psi_{m}\right) \mathrm{e}^{-\phi} \mathrm{e}^{i k \cdot X}, \\
V_{\Lambda_{3}(\mathrm{sp})}^{(-1)} & \sim \Lambda_{3}^{[m n]}\left[i \partial Z_{m} \Psi_{n}+\alpha^{\prime} k_{\mu} \psi^{\mu} \Psi_{m} \Psi_{n}\right] \mathrm{e}^{-\phi} \mathrm{e}^{i k \cdot X}, \\
V_{\Lambda_{4}(\mathrm{sp})}^{(-1)} & \sim \Lambda_{4 \mu}^{m}\left(i \partial X^{\mu} \Psi_{m}-i \partial Z_{m} \psi^{\mu}-2 \alpha^{\prime} k_{\nu} \psi^{\mu} \psi^{\nu} \Psi_{m}\right) \mathrm{e}^{-\phi} \mathrm{e}^{i k \cdot X}, \quad k^{\mu} \Lambda_{4 \mu}^{m}=0 .
\end{aligned}
$$

Each spurious state corresponds to a gauge freedom. The first one 4.9 admits to gauge away the longitudinal component of the rank two tensor $\alpha_{\mu \nu}$ whereas the second one 4.10) identifies the antisymmetric part $\alpha_{[\mu \nu]}$ together with the longitudinal three-form $e_{\mu \nu \lambda} \sim k_{[\mu} \Sigma_{\nu \lambda]}$ as unphysical. Similarly, 4.12), 4.13 and 4.14 eliminate the longitudinal components of $\left(\beta_{\mu}^{m}+\gamma_{\mu}^{m}\right), d_{\mu}^{m n}$ and $\omega_{\mu \nu}^{m}$ as well as the antisymmetric parts $\beta_{\mu}^{m}-\gamma_{\mu}^{m}$ and $\zeta_{[m n]}$. The trace of $\alpha_{\mu \nu}$ can be gauged away using (4.11).

Once the three- and two-forms $e_{\mu \nu \lambda}$ and $\omega_{\mu \nu}^{k}$ are reduced to there transverse part, contraction with $\varepsilon^{\mu \nu \lambda \rho} k_{\rho}$ dualizes them to a scalar and a vector, respectively. As we will see below, supersymmetry suggests to include these dualized states into the complex combinations (4.5) and (4.6).

\section{$4.2 \mathrm{R}$ sector}

In the $\mathrm{R}$ sector, the SCFT operators of appropriate weight give rise to a vertex operator ansatz with six wavefunctions:

$$
\begin{aligned}
V^{\left(-\frac{1}{2}\right)}= & \left(v_{\mu, I}^{a} i \partial X^{\mu} S_{a} \Sigma^{I}+\bar{\rho}_{\dot{b}, I}^{\mu} S_{\mu}^{\dot{b}} \Sigma^{I}+u_{I}^{a} \partial S_{a} \Sigma^{I}\right. \\
& \left.+y_{I}^{a} S_{a} \partial \Sigma^{I}+\bar{r}_{m, \bar{b}}^{\bar{J}} i \partial Z^{m} S^{\dot{b}} \bar{\Sigma}_{\bar{J}}+s_{m}^{a, \bar{J}} S_{a} \bar{\Sigma}_{\bar{J}}^{m}\right) \mathrm{e}^{-\phi / 2} \mathrm{e}^{i k \cdot X}
\end{aligned}
$$

The same set of states also exists with opposite chiralities with respect to both $S O(1,3)$ and $S O(6)$ (e.g. $v_{\mu, I}^{a} S_{a} \Sigma^{I} \leftrightarrow \bar{v}_{\mu, \dot{J}}^{\bar{J}} S^{\dot{b}} \bar{\Sigma}_{\bar{J}}$ ). However, the BRST constraints for the polarizations in 4.15 decouple from those of the other chirality sector which we did not display, so the discussion will be limited to the six wavefunctions shown in 4.15 for the moment. The full list of physical and 
spurious states follows from doubling the solutions of the on-shell constraints. Imposing invariance under $Q_{1}$ yields the following three independent constraints:

$$
\begin{aligned}
& 0=2 \alpha^{\prime} v_{I}^{\mu, a} \not k_{a \dot{b}}+\sqrt{2} \bar{\rho}_{\dot{b}, I}^{\mu}+\frac{1}{2} u_{I}^{a} \sigma_{a \dot{b}}^{\mu}, \\
& 0=2 \alpha^{\prime} \bar{r}_{m, \dot{b}}^{\bar{J}} \not k^{\dot{b} a}+\sqrt{2} s_{m}^{a, \bar{J}}-\frac{1}{2} y_{I}^{a} \gamma_{m}^{I \bar{J}}, \\
& 0=k_{\mu} \bar{\rho}_{\dot{b}, I}^{\mu}+\frac{1}{2 \sqrt{2}} \bar{r}_{m, \bar{b}}^{\bar{J}} \bar{\gamma}_{\bar{J} I}^{m} .
\end{aligned}
$$

The first two equations can be further disentangled into a trace and a traceless part with respect to the $\sigma^{\mu}$ and $\gamma_{m}$ matrices. Since excited spin fields are $\sigma$ and $\gamma$ traceless, the associated wavefunctions satisfy $\bar{\rho}_{\dot{b}, I}^{\mu} \bar{\sigma}_{\mu}^{\dot{b} a}=s_{m}^{a, \bar{J}} \bar{\gamma}_{\bar{J} I}^{m}=0$ by construction. Hence, the aforementioned projections simplify the BRST constraints to

$$
\begin{aligned}
u_{I}^{a} & =\alpha^{\prime} v_{\mu, I}^{b}\left(\not k \bar{\sigma}^{\mu}\right)_{b}{ }^{a} \\
\bar{\rho}_{\dot{b}, I}^{\mu} & =-\sqrt{2} \alpha^{\prime}\left(v_{I}^{\mu, a} \not k_{a \dot{b}}+\frac{1}{4} v_{\lambda, I}^{a}\left(\not k \bar{\sigma}^{\lambda} \sigma^{\mu}\right)_{a \dot{b}}\right) \\
y_{I}^{a} & =-\frac{2 \alpha^{\prime}}{3} \bar{r}_{m, \dot{b}}^{\bar{J}} \bar{\gamma}_{\bar{J} I}^{m} \not k^{\not b a} \\
s_{m}^{a, \bar{J}} & =-\sqrt{2} \alpha^{\prime}\left(\bar{r}_{m, \dot{b}}^{\bar{J}} \not k^{\dot{b} a}+\frac{1}{6} \bar{r}_{n, \dot{b}}^{\bar{I}}\left(\bar{\gamma}^{n} \gamma_{m}\right)_{\bar{I}}^{\bar{J}} \not k^{\dot{b} a}\right) \\
\bar{r}_{m, \bar{J}}^{\bar{J}} \bar{\gamma}_{\bar{J} I}^{m} & =2 \alpha^{\prime} k_{\mu} v_{I}^{\mu, a} \not k_{a \dot{b}}-v_{\mu, I}^{a} \sigma_{a \dot{b}}^{\mu}
\end{aligned}
$$

where $\bar{\rho}, u, y$ and $s$ are expressed in terms of $v$ and $\bar{r}$. It turns out that both spin $3 / 2$ and spin $1 / 2$ components of the vector spinors $v_{I}$ as well as the $\gamma$ traceless components of $\bar{r}$ give rise to an independent physical solution. The former is the $D=4$ analogue of the ten-dimensional spin $3 / 2$ state 2.22). But additionally, we find spin 1/2 Dirac fermions $\left(a^{b}, \bar{r}_{m, a}^{\bar{I}}\right)$ - both in the fundamental spinor- and in the spin $3 / 2$ representations of the R-symmetry $S O(6)$. To summarize the physical states built from 4.15 and its opposite chirality counterpart:

- eight transverse and $\sigma$ traceless spin $3 / 2$ vector spinors

$$
\begin{aligned}
V_{\chi}^{\left(-\frac{1}{2}\right)} & =\frac{1}{\sqrt{2} \alpha^{1 / 4}} \chi_{\mu, I}^{a}\left(i \partial X^{\mu} S_{a}-\sqrt{2} \alpha^{\prime} \not k_{a \dot{b}} S^{\mu \dot{b}}\right) \Sigma^{I} \mathrm{e}^{-\phi / 2} \mathrm{e}^{i k \cdot X} \\
V_{\bar{\chi}}^{\left(-\frac{1}{2}\right)} & =\frac{1}{\sqrt{2} \alpha^{\prime 1 / 4}} \bar{\chi}_{\mu, \dot{a}}^{\bar{I}}\left(i \partial X^{\mu} S^{\dot{a}}-\sqrt{2} \alpha^{\prime} \not k^{\dot{a} b} S_{b}^{\mu}\right) \bar{\Sigma}_{\bar{I}} \mathrm{e}^{-\phi / 2} \mathrm{e}^{i k \cdot X} \\
0 & =k^{\mu} \chi_{\mu, I}^{a}=\chi_{\mu, I}^{a} \sigma_{a \dot{b}}^{\mu}=k_{\mu} \bar{\chi}_{\dot{b}}^{\mu, \bar{J}}=\bar{\chi}_{\dot{b}}^{\mu, \bar{J}} \bar{\sigma}_{\mu}^{\dot{b} a}
\end{aligned}
$$

- 48 spin $1 / 2$ fermions (eight in the fundamental and 40 in spin $3 / 2$ representations of $S O(6)$ )

$$
V_{a}^{\left(-\frac{1}{2}\right)}=\frac{\alpha^{\prime 1 / 4}}{2} a_{I}^{b}\left(\left(\sigma_{\mu} \not k\right)_{b}{ }^{a} S_{a} i \partial X^{\mu}-4 \partial S_{b}\right) \Sigma^{I} \mathrm{e}^{-\phi / 2} \mathrm{e}^{i k \cdot X}
$$




$$
\begin{aligned}
V_{\bar{a}}^{\left(-\frac{1}{2}\right)} & =\frac{\alpha^{1 / 4}}{2} \bar{a}_{\dot{b}}^{\bar{I}}\left(\left(\bar{\sigma}_{\mu} \not k\right)^{\dot{b}}{ }_{\dot{a}} S^{\dot{a}} i \partial X^{\mu}-4 \partial S^{\dot{b}}\right) \bar{\Sigma}_{\bar{I}} \mathrm{e}^{-\phi / 2} \mathrm{e}^{i k \cdot X}, \\
V_{r}^{\left(-\frac{1}{2}\right)} & =\frac{1}{\sqrt{2} \alpha^{\prime 1 / 4}} r_{m, I}^{a}\left(i \partial Z^{m} \Sigma^{I} S_{a}-\sqrt{2} \alpha^{\prime} \not k_{a \dot{b}} S^{\dot{b}} \Sigma^{m, I}\right) \mathrm{e}^{-\phi / 2} \mathrm{e}^{i k \cdot X}, \\
V_{\bar{r}}^{\left(-\frac{1}{2}\right)} & =\frac{1}{\sqrt{2} \alpha^{\prime 1 / 4}} \bar{r}_{m, \dot{a}}^{\bar{I}}\left(i \partial Z^{m} \bar{\Sigma}_{\bar{I}} S^{\dot{a}}-\sqrt{2} \alpha^{\prime} \not k^{\dot{a} b} S_{b} \bar{\Sigma}_{\bar{I}}^{m}\right) \mathrm{e}^{-\phi / 2} \mathrm{e}^{i k \cdot X} .
\end{aligned}
$$

The following spurious solutions have been subtracted to remove internal derivatives $\partial \Sigma^{I}$ from the vertex operators:

$$
\begin{aligned}
V_{\Theta(\mathrm{sp})}^{\left(-\frac{1}{2}\right)} \sim & \Theta_{I}^{a}\left[\left(\not k_{a \dot{b}} \bar{\sigma}_{\mu}^{\dot{b} b}+4 k_{\mu} \delta_{a}^{b}\right) i \partial X^{\mu} S_{b} \Sigma^{I}-2 \sqrt{2}\left(\alpha^{\prime} k^{\mu} \not k_{a \dot{b}}+\frac{1}{4} \sigma_{a \dot{b}}^{\mu}\right) S_{\mu}^{\dot{b}} \Sigma^{I}\right. \\
& \left.+6 \partial S_{a} \Sigma^{I}+4 S_{a} \partial \Sigma^{I}+\not k_{a \dot{b}} \gamma_{m}^{I \bar{J}} i \partial Z^{m} S^{\dot{b}} \bar{\Sigma}_{\bar{I}}\right] \mathrm{e}^{-\phi / 2} \mathrm{e}^{i k \cdot X}, \\
V_{\bar{\Theta}(\mathrm{sp})}^{\left(-\frac{1}{2}\right)} \sim & \bar{\Theta}_{\dot{b}}^{\bar{I}}\left[\left(\not k^{\dot{b} a} \sigma_{a \dot{a}}^{\mu}+4 k^{\mu} \delta_{\dot{a}}^{\dot{b}}\right) i \partial X_{\mu} S^{\dot{a}} \bar{\Sigma}_{\bar{I}}-2 \sqrt{2}\left(\alpha^{\prime} k_{\mu} \not k^{\dot{b} a}+\frac{1}{4} \bar{\sigma}_{\mu}^{\dot{b} a}\right) S_{a}^{\mu} \bar{\Sigma}_{\bar{I}}\right. \\
& \left.+6 \partial S^{\dot{b}} \bar{\Sigma}_{\bar{I}}+4 S^{\dot{b}} \partial \bar{\Sigma}_{\bar{I}}+\not k^{\dot{b} a} \gamma_{\bar{I} J}^{m} i \partial Z_{m} S_{a} \Sigma^{J}\right] \mathrm{e}^{-\phi / 2} \mathrm{e}^{i k \cdot X} .
\end{aligned}
$$

They are the dimensional reduction of the ten-dimensional spurious state (2.23).

\subsection{SUSY transformations}

Now with all the higher order OPEs and physical spectrum in hands, we are able to compute the SUSY transformations by acting with the supercharge operators on the physical states and evaluating the corresponding contour integral.

In $\mathcal{N}=4$ SUSY, the SUSY parameters $\eta_{I}^{a}, \bar{\eta}_{\dot{a}}^{\bar{I}}$ are chiral spinors of both the $S O(1,3)$ Lorentz group and the internal $S O(6)$ R-symmetry group. For our convenience, we choose these SUSY parameters to have mass dimension $\left[M^{-\frac{1}{2}}\right]$. As we verify case by case, action of the supercharges $\mathcal{Q}_{a}^{I}$ and $\overline{\mathcal{Q}}_{\bar{J}}^{\dot{b}}$ given by 3.14 and 3.18 , 3.19 takes bosonic (fermionic) vertex operators exactly into fermionic (bosonic) vertex operators, including their couplings. The polarization wavefunction of the $\mathcal{Q}$ image state is expressed in terms of $\eta_{I}^{a}, \bar{\eta}_{\dot{a}}^{\bar{I}}$ and the pre-image wavefunction 13

Once we perform the SUSY variations, besides physical fields in the spectrum, we will also get certain spurious states. As an example, let us consider the anti-supercharge acting on the spin- $\frac{3}{2}$ fermionic state $\chi_{\mu, I}^{a}$. Evaluating the contour integral yields

$$
\left[\bar{\eta}_{\dot{a}}^{\bar{I}} \overline{\mathcal{Q}}_{\bar{I}}^{\left(-\frac{1}{2}\right), \dot{a}}, V_{\chi}^{\left(-\frac{1}{2}\right)}\right]=V_{\alpha}^{(-1)}\left(\alpha_{\mu \nu}=\frac{1}{\sqrt{2}} \bar{\eta}_{\dot{a}}^{\bar{I}}\left(\bar{\sigma}_{(\mu}^{\dot{a} a} \chi_{\nu), a, I}+\alpha^{\prime} k^{\dot{a} a} k_{(\mu} \chi_{\nu), a, I}\right) C_{\bar{I}}^{I}\right)
$$

\footnotetext{
${ }^{13}$ In our settings, all the wavefunctions of bosonic fields have mass dimension 0 , and all the wavefunction of fermionic fields have mass dimension $\frac{1}{2}$, see appendix $\mathrm{C}$ for their explicit construction in terms of (massive) spinor helicity variables.
} 


$$
\begin{aligned}
& +V_{d}^{(-1)}\left(d_{\mu}^{[m n]}=-\frac{\sqrt{\alpha^{\prime}}}{4} \bar{\eta}_{\dot{a}}^{\bar{I}} k^{\dot{\alpha} a} \chi_{\mu, a, J}\left(\gamma^{[m} \gamma^{n]} C\right)_{\bar{I}}^{J}\right) \\
& +V_{\pi(\mathrm{sp})}^{(-1)}\left(\pi_{\mu}=-\frac{\sqrt{\alpha^{\prime}}}{4} \bar{\eta}_{\dot{a}}^{\bar{I}} \not k^{\dot{a} a} \chi_{\mu, a, I} C_{\bar{I}}^{I}\right) \\
& +V_{\Sigma(\mathrm{sp})}^{(-1)}\left(\Sigma_{[\mu \nu]}=-\frac{1}{\sqrt{2}} \bar{\eta}_{\dot{a}}^{\bar{I}}\left(\bar{\sigma}_{[\mu}^{\dot{a} a} \chi_{\nu], a, I}-\alpha^{\prime} k^{\dot{a} a} k_{[\mu} \chi_{\nu], a, I}\right) C_{\bar{I}}^{I}\right) .
\end{aligned}
$$

As we can see, we obtain two physical states - a spin two boson $\alpha_{\mu \nu}$ and a vector $d_{\mu}^{[m n]}$ from (4.3) and (4.4), plus two spurious states $-\pi_{\mu}$ and $\Sigma_{[\mu \nu]}$, see 4.9 and 4.10 for their full vertex operators. We will drop out all these spurious states in our final results for simplicity.

All the physical states form one big supermultiplet of $\mathcal{N}=4$. The structure of the explicit SUSY variations listed in this section is summarized in figure 5 below. This diagram will be refined in section 7 to take helicity quantum numbers into account.

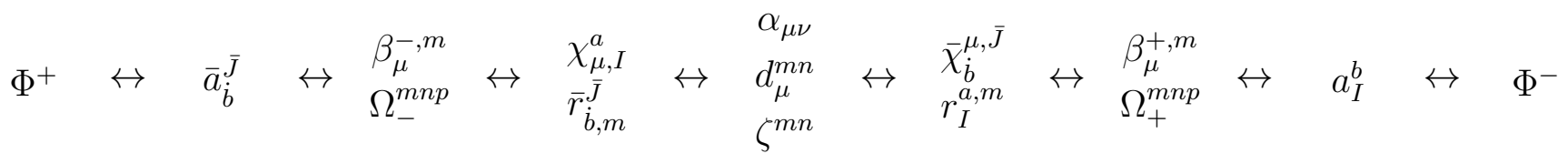

Figure 5: $\mathcal{N}=4$ SUSY multiplet: action of the left-handed SUSY charge $\mathcal{Q}_{a}^{I}$ transforms a state into (a combination of) its left neighbors, whereas $\overline{\mathcal{Q}}_{\bar{J}}^{\dot{b}}$ action maps states into right neighbors.

The pattern of SUSY variations depicted in figure 5 justifies the complex combinations (4.5) of vectors and 4.9) of scalars: The complex conjugates appear on widely separated positions of the multiplet (i.e. the $\beta^{+}$and $\beta^{-}$are separated by four $\mathcal{Q}$ actions whereas $\Phi^{+} \leftrightarrow \Phi^{-}$requires eight supercharge applications). Also, the internal scalar $\Omega^{m n p}$ splits into self-dual and anti-self-dual components $\Omega_{ \pm}^{m n p}$ which sit at different points of the multiplet.

There are group theoretic selection rules for the possible outcome of a physical state's SUSY variations, based on the $S O(1,3) \times S O(6)$ symmetry. Firstly, according to its eigenvalue under diagonal Lorentz currents, $\mathcal{Q}$ can only change the spin by $\pm \frac{1}{2}$. Secondly, transformations have to compatible with the $S O(6)$ quantum numbers involved. Representation of the $S O(6) \equiv S U(4)$ R-symmetry group are referred to using their Dynkin Labels $[k, p, q]{ }^{14}$ The SUSY variation of a state $\in[k, p, q]$ aligns into the tensor product with $[0,1,0] \ni \mathcal{Q}^{I}$ or $[0,0,1] \ni \overline{\mathcal{Q}}_{\bar{J}}$ of the SUSY

\footnotetext{
${ }^{14}$ Our conventions for the Dynkin labels $[k, p, q]$ are such that $[1,0,0]$ labels the vector representation, and $[0,1,0]$ and $[0,0,1]$ are left- and right-handed spinor. A generic representation with labels $[k, p, q]$ has dimension

$$
D_{[k, p, q]}=\frac{1}{12}(k+p+q+3)(k+p+2)(k+q+2)(k+1)(p+1)(q+1),
$$




\begin{tabular}{|c|c|c||c|c|c|}
\hline Spin & Wavefunctions & $S O(6)$ rep. & Spin & Wavefunctions & $S O(6)$ rep. \\
\hline \hline 2 & $\alpha_{\mu \nu}$ & {$[0,0,0]$} & $3 / 2$ & $\chi_{\mu, I}^{a}$ & {$[0,1,0]$} \\
\hline 1 & $\beta_{\mu}^{ \pm, m}$ & {$[1,0,0]$} & $3 / 2$ & $\bar{\chi}_{\dot{b}}^{\mu, \bar{J}}$ & {$[0,0,1]$} \\
\hline 1 & $d_{\mu}^{[m n]}$ & {$[0,1,1]$} & $1 / 2$ & $r_{m, I}^{a}$ & {$[1,1,0]$} \\
\hline 0 & $\zeta^{(m n)}$ & {$[2,0,0]$} & $1 / 2$ & $\bar{r}_{\dot{b}}^{m, \bar{J}}$ & {$[1,0,1]$} \\
\hline 0 & $\Omega_{m n l}^{+}$ & {$[0,2,0]$} & $1 / 2$ & $a_{I}^{b}$ & {$[0,1,0]$} \\
\hline 0 & $\Omega_{m n l}^{-}$ & {$[0,0,2]$} & $1 / 2$ & $\bar{a}_{\dot{b}}^{\bar{J}}$ & {$[0,0,1]$} \\
\hline 0 & $\Phi^{ \pm}$ & {$[0,0,0]$} & & & \\
\hline
\end{tabular}

Table 1: R-symmetry content of the massive $\mathcal{N}=4$ multiplet in $S O(6)$ Dynkin label notation

charge. Table 1 gives an overview of the R-symmetry representations involved (see the following subsection for the $\Omega^{ \pm}$splitting).

\subsubsection{SUSY transformation of bosonic states}

In this subsubsection, we will analyze supercharge acting on the bosonic states. The spin two field $\alpha_{\mu \nu}$ transforms into left- and right-handed spin- $\frac{3}{2}$ fermions $\mathcal{Q} \alpha \rightarrow \chi$ and $\overline{\mathcal{Q}} \alpha \rightarrow \bar{\chi}$ in lines with $[0,0,0] \otimes[0,1,0] \rightarrow[0,1,0]$ for the R-symmetry scalar $\alpha_{\mu \nu}$. The SUSY variations of this field are parallel to 2.27) in ten dimensions:

$$
\begin{aligned}
& {\left[\eta_{I}^{a} \mathcal{Q}_{a}^{\left(+\frac{1}{2}\right), I}, V_{\alpha}^{(-1)}\right]=V_{\chi}^{\left(-\frac{1}{2}\right)}\left(\chi_{\mu, I}^{b}=\frac{1}{\sqrt{2}} \eta_{I}^{a} \alpha_{\mu \nu}\left(\not k \bar{\sigma}^{\nu}\right)_{a}^{b}\right),} \\
& {\left[\bar{\eta}_{\dot{a}}^{\bar{I}} \overline{\mathcal{Q}}_{\bar{I}}^{\left(+\frac{1}{2}\right), \dot{a}}, V_{\alpha}^{(-1)}\right]=V_{\bar{\chi}}^{\left(-\frac{1}{2}\right)}\left(\bar{\chi}_{\mu, \dot{a}}^{\bar{I}}=\frac{1}{\sqrt{2}} \bar{\eta}_{\dot{a}}^{\bar{I}} \alpha_{\mu \nu}\left(\not k \sigma^{\nu}\right)^{\dot{a}}{ }_{\dot{b}}\right) .}
\end{aligned}
$$

The spin one fields fall into vector and two-form representations $[1,0,0]$ and $[0,1,1]$ of the $\mathrm{R}$ symmetry, so their SUSY image belongs to $[0,1,0] \otimes[1,0,0] \rightarrow[1,1,0] \oplus[0,0,1]$ and $[0,1,0] \otimes$ $[0,1,1] \rightarrow[0,1,0] \oplus[0,2,1] \oplus[1,0,1]$, respectively (note that $[0,2,1]$ does not occur in our multiplet). This implies that $\beta_{\mu}^{ \pm, m}$ can transform into an internal left-handed fermion $r_{m, I}^{a} \in[1,1,0]$, and rightand tensor products act as follows on Dynkin labels:

$$
\begin{aligned}
{[k, p, q] \otimes[0,1,0]=} & {[k, p, q-1] \oplus[k, p+1, q] \oplus[k+1, p-1, q] \oplus[k-1, p, q+1], } \\
{[k, p, q] \otimes[0,0,1]=} & {[k, p, q+1] \oplus[k, p-1, q] \oplus[k+1, p, q-1] \oplus[k-1, p+1, q], } \\
{[k, p, q] \otimes[1,0,0]=} & {[k, p+1, q-1] \oplus[k, p-1, q+1] \oplus[k+1, p, q] } \\
& \oplus[k+1, p-1, q-1] \oplus[k-1, p, q] \oplus[k-1, p+1, q+1] .
\end{aligned}
$$


handed spin- $\frac{3}{2}$ fermions $\bar{\chi}_{\mu \dot{a}}^{\bar{I}}$ or a spin- $\frac{1}{2}$ fermions $\bar{a}_{\dot{b}}^{\bar{I}}$, in short: $\mathcal{Q} \beta^{ \pm} \rightarrow \bar{\chi}+\bar{a}+r$. For the $S O(6)$ two-form $d^{[m n]}$, we will get the opposite chirality configuration, $\mathcal{Q} d \rightarrow \chi+a+\bar{r}$. The explicit results for the left-handed $\mathcal{Q}_{a}^{I}$ are given as follows ${ }^{15}$

$$
\begin{aligned}
{\left[\eta_{I}^{b} \mathcal{Q}_{b}^{\left(+\frac{1}{2}\right), I}, V_{\beta^{+}}^{(-1)}\right] } & =V_{\bar{\chi}}^{\left(-\frac{1}{2}\right)}\left(\bar{\chi}_{\mu, \dot{I}}^{\bar{I}}=\frac{1}{3 \sqrt{2}} \eta_{I}^{b}\left[3 \beta_{\mu}^{+, m} \not k_{b \dot{b}}-k_{\mu} \beta_{b \dot{b}}^{+, m}-\left(\beta^{+, m} \not k \sigma_{\mu}\right)_{b \dot{b}}\right] \gamma_{m}^{I \bar{I}}\right) \\
& +V_{r}^{\left(-\frac{1}{2}\right)}\left(r_{n, J}^{c}=-\frac{1}{6 \sqrt{2}} \eta_{I}^{b}\left(\beta^{+, m} \not k\right)_{b}{ }^{c}\left[6 \delta_{m n}^{(6)} \delta_{J}^{I}+\left(\gamma_{m} \bar{\gamma}_{n}\right)_{J}^{I}\right]\right), \\
{\left[\eta_{I}^{b} \mathcal{Q}_{b}^{\left(+\frac{1}{2}\right), I}, V_{\beta^{-}}^{(-1)}\right] } & =V_{\bar{a}}^{\left(-\frac{1}{2}\right)}\left(\bar{a}_{\dot{b}}^{\bar{I}}=-\frac{1}{2 \sqrt{\alpha^{\prime}}} \eta_{I}^{b} \beta_{b \dot{b}}^{-, m} \gamma_{m}^{I \bar{I}}\right), \\
{\left[\eta_{I}^{b} \mathcal{Q}_{b}^{\left(+\frac{1}{2}\right), I}, V_{d}^{(-1)}\right] } & =V_{\chi}^{\left(-\frac{1}{2}\right)}\left(\chi_{\mu, J}^{c}=\frac{1}{6 \sqrt{\alpha^{\prime}}} \eta_{I}^{b}\left[3 d_{\mu}^{m n} \delta_{b}^{c}+\left(\not d^{m n} \bar{\sigma}_{\mu}+\alpha^{\prime} k_{\mu} \phi^{m n} \not k\right)_{b}{ }^{c}\right]\left(\gamma_{m} \bar{\gamma}_{n}\right)^{I}{ }_{J}\right) \\
& +V_{\bar{r}}^{\left(-\frac{1}{2}\right)}\left(\bar{r}_{l, \dot{b}}^{\bar{I}}=\frac{1}{6 \sqrt{\alpha^{\prime}}} \eta_{I}^{b} \phi_{b \dot{b}}^{m n} \gamma_{n}^{I \bar{I}}\left[6 \delta_{m l}^{(6)} \delta_{\bar{I}}^{\bar{J}}+\left(\bar{\gamma}_{m} \gamma_{l}\right)_{\bar{I}}^{\bar{J}}\right]\right),
\end{aligned}
$$

whereas the action of right-handed $\overline{\mathcal{Q}}_{\bar{J}}^{\dot{b}}$ yields

$$
\begin{aligned}
{\left[\bar{\eta}_{\dot{b}}^{\bar{I}} \overline{\mathcal{Q}}_{\bar{I}}^{\left(+\frac{1}{2}\right), \dot{b}}, V_{\beta^{+}}^{(-1)}\right] } & =V_{a}^{\left(-\frac{1}{2}\right)}\left(a_{I}^{b}=-\frac{1}{2 \sqrt{\alpha^{\prime}}} \bar{\eta}_{\dot{i}}^{\bar{I}} \beta^{+, m, \dot{b} b} \bar{\gamma}_{m, \bar{I} I}\right) \\
{\left[\bar{\eta}_{\dot{b}}^{\bar{I}} \overline{\mathcal{Q}}_{\bar{I}}^{\left(+\frac{1}{2}\right), \dot{b}}, V_{\beta^{-}}^{(-1)}\right] } & =V_{\chi}^{\left(-\frac{1}{2}\right)}\left(\chi_{\mu, I}^{b}=\frac{1}{3 \sqrt{2}} \bar{\eta}_{\dot{b}}^{\bar{I}}\left[3 \beta_{\mu}^{-, m} \not k^{\dot{b} b}-k_{\mu} \beta^{-, m, \dot{b} b}-\left(\not \beta^{-, m} \not k \bar{\sigma}_{\mu}\right)^{\dot{b} b}\right] \bar{\gamma}_{m, \bar{I} I}\right) \\
& +V_{\bar{r}}^{\left(-\frac{1}{2}\right)}\left(\bar{r}_{n, \dot{c}}^{\bar{J}}=-\frac{1}{6 \sqrt{2}} \bar{\eta}_{\dot{b}}^{\bar{I}}\left(\not \beta^{-, m} \not k\right)_{\dot{c}}^{\dot{b}}\left[6 \delta_{m n}^{(6)} \delta_{\bar{I}}^{\bar{J}}+\left(\bar{\gamma}_{m} \gamma_{n}\right)_{\bar{I}}^{\bar{J}}\right]\right) \\
{\left[\bar{\eta}_{\dot{b}}^{\bar{I}} \overline{\mathcal{Q}}_{\bar{I}}^{\left(+\frac{1}{2}\right), \dot{b}}, V_{d}^{(-1)}\right] } & =V_{\bar{\chi}}^{\left(-\frac{1}{2}\right)}\left(\bar{\chi}_{\mu, \dot{c}}^{\bar{J}}=\frac{1}{6 \sqrt{\alpha^{\prime}}} \bar{\eta}_{\dot{b}}^{\bar{I}}\left[3 d_{\mu}^{m n} \delta_{\dot{c}}^{\dot{b}}+\left(\not d^{m n} \sigma_{\mu}+\alpha^{\prime} k_{\mu} \not \phi k\right)_{\dot{c}}^{\dot{b}}\right]\left(\bar{\gamma}_{m} \gamma_{n}\right)_{\bar{I}}^{\bar{J}}\right) \\
& +V_{r}^{\left(-\frac{1}{2}\right)}\left(r_{J, p}^{b}=\frac{1}{6 \sqrt{\alpha^{\prime}}} \bar{\eta}_{\dot{b}}^{\bar{I}} \phi^{m n, \dot{b} b} \bar{\gamma}_{n, \bar{I} I}\left[6 \delta_{m p}^{(6)} \delta_{J}^{I}+\left(\gamma_{m} \bar{\gamma}_{p}\right)_{J}^{I}\right]\right) .
\end{aligned}
$$

Then we are left with the $S O(1,3)$ scalar fields $\Phi^{ \pm}, \zeta^{(m n)}$ and $\Omega_{m n l}$. The internal states $\Omega_{m n l}$ represent both self-dual and anti-self-dual three-forms of $S O(6)$. We will denote their irreducible components as $\Omega_{m n l}^{+} \in[0,2,0]$ and $\Omega_{m n l}^{-} \in[0,0,2]$, for the self-dual and anti-self-dual part, respectively. Their defining irreducibility constraint is

$$
\Omega_{m n l}^{-}\left(\gamma^{m n l}\right)_{I \bar{I}}=\Omega_{m n l}^{+}\left(\bar{\gamma}^{m n l}\right)^{\bar{I} I}=0
$$

The $S O(6)$ selection rules constrain $\mathcal{Q}^{I} \zeta^{(m n)} \in[0,1,0] \otimes[2,0,0] \rightarrow[2,1,0] \oplus[1,0,1]$ as well as $\mathcal{Q}^{I} \Omega_{m n l}^{+} \in[0,1,0] \otimes[0,2,0] \rightarrow[0,3,0] \oplus[1,1,0]$ and $\mathcal{Q}^{I} \Omega_{m n l}^{-} \in[0,1,0] \otimes[0,0,2] \rightarrow[0,1,2] \oplus[0,0,1]$. Thus, we expect the internal spin- $\frac{1}{2}$ fermion $\bar{r}$ or $r$ by performing the SUSY transformation $\mathcal{Q} \zeta \rightarrow \bar{r}$,

\footnotetext{
${ }^{15}$ There is a subtlety in these computations (and also for some later ones) related to the fact that gamma matrices associated with spacetime and internal dimensions are anticommuting.
} 
and $\overline{\mathcal{Q}} \zeta \rightarrow r$. Three-forms, on the other hand, are mapped to either $r$ or $\bar{a}$, depending on the self-duality property $\mathcal{Q} \Omega^{+} \rightarrow r$ or $\mathcal{Q} \Omega^{-} \rightarrow \bar{a}$. The supercharges acting on $\Phi^{ \pm}$and $\zeta^{(m n)}$ yield

$$
\begin{gathered}
{\left[\eta_{I}^{b} \mathcal{Q}_{b}^{\left(+\frac{1}{2}\right), I}, V_{\Phi^{+}}^{(-1)}\right]=0, \quad\left[\bar{\eta}_{\dot{b}}^{\bar{I}} \overline{\mathcal{Q}}_{\bar{I}}^{\left(+\frac{1}{2}\right), \dot{b}}, V_{\Phi^{-}}^{(-1)}\right]=0,} \\
{\left[\bar{\eta}_{\dot{b}}^{\bar{I}} \overline{\mathcal{Q}}_{\bar{I}}^{\left(+\frac{1}{2}\right), \dot{b}}, V_{\Phi^{+}}^{(-1)}\right]=V_{\bar{a}}^{\left(-\frac{1}{2}\right)}\left(\bar{a}_{\dot{b}}^{\bar{I}}=-\alpha^{\prime-\frac{1}{2}} \Phi^{+} \bar{\eta}_{\dot{b}}^{\bar{I}}\right),} \\
{\left[\eta_{I}^{b} \mathcal{Q}_{b}^{\left(+\frac{1}{2}\right), I}, V_{\Phi^{-}}^{(-1)}\right]=V_{a}^{\left(-\frac{1}{2}\right)}\left(a_{I}^{b}=-\alpha^{\prime-\frac{1}{2}} \Phi^{-} \eta_{I}^{b}\right),}
\end{gathered}
$$

and

$$
\begin{aligned}
& {\left[\eta_{I}^{b} \mathcal{Q}_{b}^{\left(+\frac{1}{2}\right), I}, V_{\zeta}^{(-1)}\right]=V_{\bar{r}}^{\left(-\frac{1}{2}\right)}\left(\bar{r}_{\dot{b}}^{m, \bar{I}}=\frac{1}{\sqrt{2}} \eta_{I}^{b} \zeta^{(m n)} \not k_{b \dot{b}} \gamma_{n}^{I \bar{I}}\right)} \\
& {\left[\bar{\eta}_{\dot{b}}^{\bar{I}} \overline{\mathcal{Q}}_{\bar{I}}^{\left(+\frac{1}{2}\right), \dot{b}}, V_{\zeta}^{(-1)}\right]=V_{r}^{\left(-\frac{1}{2}\right)}\left(r_{I}^{m, b}=\frac{1}{\sqrt{2}} \bar{\eta}_{\dot{b}}^{\bar{I}} \zeta^{(m n)} \not k^{\dot{b} b} \bar{\gamma}_{n, \bar{I} I}\right) .}
\end{aligned}
$$

On the three-forms $\Omega_{m n l}^{ \pm}$, we obtain

$$
\begin{aligned}
& {\left[\eta_{I}^{b} \mathcal{Q}_{b}^{\left(+\frac{1}{2}\right), I}, V_{\Omega^{+}}^{(-1)}\right]=V_{r}^{\left(-\frac{1}{2}\right)}\left(r_{k, J}^{b}=-\frac{1}{4 \sqrt{\alpha^{\prime}}} \eta_{I}^{b} \Omega_{m n l}^{+}\left(\gamma_{k} \bar{\gamma}^{m n l}\right)_{J}^{I}\right),} \\
& {\left[\bar{\eta}_{\dot{b}}^{\bar{I}} \overline{\mathcal{Q}}_{\bar{I}}^{\left(+\frac{1}{2}\right), \dot{b}}, V_{\Omega^{+}}^{(-1)}\right]=V_{a}^{\left(-\frac{1}{2}\right)}\left(a_{I}^{b}=\frac{1}{2 \sqrt{2}} \bar{\eta}_{\dot{b}}^{\bar{I}} \Omega_{m n l}^{+} \not k^{\dot{b} b}\left(\bar{\gamma}^{m n l}\right)_{\bar{I} I}\right),}
\end{aligned}
$$

and

$$
\begin{aligned}
& {\left[\eta_{I}^{b} \mathcal{Q}_{b}^{\left(+\frac{1}{2}\right), I}, V_{\Omega^{-}}^{(-1)}\right]=V_{\bar{a}}^{\left(-\frac{1}{2}\right)}\left(\bar{a}_{\dot{b}}^{\bar{I}}=\frac{1}{2 \sqrt{2}} \eta_{I}^{b} \Omega_{m n l}^{-} k_{b \dot{b}}\left(\gamma^{m n l}\right)^{I \bar{I}}\right),} \\
& {\left[\bar{\eta}_{\dot{b}}^{\bar{I}} \overline{\mathcal{Q}}_{\bar{I}}^{\left(+\frac{1}{2}\right), \dot{b}}, V_{\Omega^{-}}^{(-1)}\right]=V_{\bar{r}}^{\left(-\frac{1}{2}\right)}\left(\bar{r}_{k, \dot{b}}^{\bar{J}}=-\frac{1}{4 \sqrt{\alpha^{\prime}}} \bar{\eta}_{\dot{b}}^{\bar{I}} \Omega_{m n l}^{-}\left(\bar{\gamma}_{k} \gamma^{m n l}\right)_{\bar{I}}^{\bar{J}}\right) .}
\end{aligned}
$$

\subsubsection{SUSY transformation of fermionic states}

In this subsubsection, we investigate the (anti-)supercharge acting on the fermionic states. Following the strategy outlined before, we first derive a selection rule from group theory and then perform SUSY variations to get the expression of the bosonic wavefunctions explicitly. All the transformations are symmetric under simultaneous exchange of chiralities on the supercharges and the states (where $\Phi^{+}, \beta^{+}, \Omega^{+} \leftrightarrow \Phi^{-}, \beta^{-}, \Omega^{-}$). We will only comment on one out of two inequivalent cases in the text but also give the formulae for the images under chirality reversal.

Since both the spin- $\frac{3}{2}$ fermions $(\chi, \bar{\chi})$ and the spin- $\frac{1}{2}$ states $(a, \bar{a})$ fall into (anti-)fundamental R-symmetry representations, the $S O(6)$ content of their SUSY variation is $[0,1,0] \otimes[0,1,0] \rightarrow$ $[0,2,0] \oplus[1,0,0]$ and $[0,0,1] \otimes[0,1,0] \rightarrow[0,0,0] \oplus[0,1,1]$. The (anti-)supercharge acting on 
$\chi_{\mu, I}^{a}\left(\bar{\chi}_{\mu, \dot{a}}^{\bar{I}}\right)$ will give us vectors $\beta_{\mu}^{ \pm, m}$. In the cases $\mathcal{Q}^{I} \bar{\chi}_{\mu, \dot{a}}^{\bar{I}}$ and $\overline{\mathcal{Q}}_{\bar{J}} \chi_{\mu, I}^{a}$ of opposite chirality, the spin two field $\alpha_{\mu \nu}$ and the vector $d_{\mu}^{[m n]}$ can emerge. Indeed,

$$
\begin{aligned}
{\left[\eta_{I}^{a} \mathcal{Q}_{a}^{\left(-\frac{1}{2}\right), I}, V_{\chi}^{\left(-\frac{1}{2}\right)}\right] } & =V_{\beta^{-}}^{(-1)}\left(\beta_{\mu}^{-, m}=\frac{1}{\sqrt{2}} \eta_{I}^{a} \chi_{\mu, a, J}\left(\gamma^{m} C\right)^{I J}\right) \\
{\left[\bar{\eta}_{\dot{a}}^{\bar{I}} \overline{\mathcal{Q}}_{\bar{I}}^{\left(-\frac{1}{2}\right), \dot{a}}, V_{\chi}^{\left(-\frac{1}{2}\right)}\right] } & =V_{\alpha}^{(-1)}\left(\alpha_{\mu \nu}=\frac{1}{\sqrt{2}} \bar{\eta}_{\dot{a}}^{\bar{I}}\left(\bar{\sigma}_{(\mu}^{\dot{a} a} \chi_{\nu), a, I}+\alpha^{\prime} \not k^{\dot{a} a} k_{(\mu} \chi_{\nu), a, I}\right) C_{\bar{I}}^{I}\right) \\
& +V_{d}^{(-1)}\left(d_{\mu}^{[m n]}=-\frac{\sqrt{\alpha^{\prime}}}{4} \bar{\eta}_{\dot{a}}^{\bar{I}} k^{\dot{a} a} \chi_{\mu, a, I}\left(\bar{\gamma}^{m n} C\right)_{\bar{I}}^{I}\right),
\end{aligned}
$$

and 16

$$
\begin{aligned}
{\left[\eta_{I}^{a} \mathcal{Q}_{a}^{\left(-\frac{1}{2}\right), I}, V_{\bar{\chi}}^{\left(-\frac{1}{2}\right)}\right] } & =V_{\alpha}^{(-1)}\left(\alpha_{\mu \nu}=\frac{1}{\sqrt{2}} \eta_{I}^{a}\left(\sigma_{(\mu|a \dot{a}|} \bar{\chi}_{\nu)}^{\dot{a}, \bar{I}}+\alpha^{\prime} \not k_{a \dot{a}} k_{(\mu} \bar{\chi}_{\nu)}^{\dot{a}, \bar{I}}\right) C_{\bar{I}}^{I}\right) \\
& +V_{d}^{(-1)}\left(d_{\mu}^{[m n]}=-\frac{\sqrt{\alpha^{\prime}}}{4} \eta_{I}^{a} \not k_{a \dot{a}} \bar{\chi}_{\mu}^{\dot{a}, \bar{I}}\left(\gamma^{m n} C\right)_{\bar{I}}^{I}\right), \\
{\left[\bar{\eta}_{\dot{a}}^{\bar{I}} \overline{\mathcal{Q}}_{\bar{I}}^{\left(-\frac{1}{2}\right), \dot{a}}, V_{\bar{\chi}}^{\left(-\frac{1}{2}\right)}\right] } & =V_{\beta^{+}}^{(-1)}\left(\beta_{\mu}^{+, m}=\frac{1}{\sqrt{2}} \bar{\eta}_{\dot{a}}^{\bar{I}} \bar{\chi}_{\mu}^{\dot{a}, \bar{J}}\left(\bar{\gamma}^{m} C\right)_{\bar{I} \bar{J}}\right) .
\end{aligned}
$$

The supercharge action on $a_{I}^{b}$ and $\bar{a}_{\dot{b}}^{\bar{I}}$ follows the same selection rules with respect to $S O(6)$ but different ones with respect to spacetime spin. The corresponding SUSY transformations read

$$
\begin{aligned}
{\left[\eta_{I}^{b} \mathcal{Q}_{b}^{\left(-\frac{1}{2}\right), I}, V_{a}^{\left(-\frac{1}{2}\right)}\right] } & =V_{\beta^{+}}^{(-1)}\left(\beta_{\mu}^{+, m}=\frac{\sqrt{\alpha^{\prime}}}{2} \eta_{I}^{b}\left[k_{\mu} \delta_{b}{ }^{c}+\left(\not k \bar{\sigma}_{\mu}\right)_{b}{ }^{c}\right] a_{c, J}\left(\gamma^{m} C\right)^{I J}\right) \\
& +V_{\Omega^{+}}^{(-1)}\left(\Omega_{m n l}^{+}=\frac{1}{12 \sqrt{2}} \eta_{I}^{b} a_{b, J}\left(\gamma_{m n l} C\right)^{I J}\right), \\
{\left[\bar{\eta}_{\dot{b}}^{\bar{I}} \overline{\mathcal{Q}}_{\bar{I}}^{\left(-\frac{1}{2}\right), \dot{b}}, V_{a}^{\left(-\frac{1}{2}\right)}\right] } & =V_{\Phi^{-}}^{(-1)}\left(\Phi^{-}=\sqrt{\alpha^{\prime}} \bar{\eta}_{\dot{b}}^{\bar{I}} k^{\dot{b} b} a_{b, I} C_{\bar{I}}^{I}\right),
\end{aligned}
$$

and

$$
\begin{aligned}
{\left[\eta_{I}^{b} \mathcal{Q}_{b}^{\left(-\frac{1}{2}\right), I}, V_{\bar{a}}^{\left(-\frac{1}{2}\right)}\right] } & =V_{\Phi^{+}}^{(-1)}\left(\Phi^{+}=\sqrt{\alpha^{\prime}} \eta_{I}^{b} \not k_{b \dot{b}} \bar{a}^{\dot{b}, \bar{I}} C_{\bar{I}}^{I}\right) \\
{\left[\bar{\eta}_{\dot{b}}^{\bar{I}} \overline{\mathcal{Q}}_{\bar{I}}^{\left(-\frac{1}{2}\right), \dot{b}}, V_{\bar{a}}^{\left(-\frac{1}{2}\right)}\right] } & =V_{\beta^{-}}^{(-1)}\left(\beta_{\mu}^{-, m}=\frac{\sqrt{\alpha^{\prime}}}{2} \bar{\eta}_{\dot{b}}^{\bar{I}}\left[k_{\mu} \delta_{\dot{c}}^{\dot{b}}+\left(\not k \sigma_{\mu}\right)_{\dot{c}}^{\dot{b}}\right] \bar{a}^{\dot{c}, \bar{J}}\left(\bar{\gamma}^{m} C\right)_{\bar{I} \bar{J}}\right) \\
& +V_{\Omega^{-}}^{(-1)}\left(\Omega_{m n l}^{-}=\frac{1}{12 \sqrt{2}} \bar{\eta}_{\dot{b}}^{\bar{I}} \bar{a}^{\dot{b}, \bar{J}^{\prime}}\left(\bar{\gamma}_{m n l} C\right)_{\bar{I} \bar{J}}\right) .
\end{aligned}
$$

Notice we do not get a vector $d_{\mu}^{[m n]}$ in the SUSY transformations, although it is allowed by $S O(1,3) \times S O(6)$.

Now we are left with the internal spin- $\frac{1}{2}$ fermions $r$ and $\bar{r}$. Group theory admits SUSY variations in $[0,1,0] \otimes[1,1,0] \rightarrow[1,2,0] \oplus[2,0,0] \oplus[0,1,1]$ and $[0,0,1] \otimes[1,1,0] \rightarrow[1,1,1] \oplus[1,0,0] \oplus[0,2,0]$

\footnotetext{
${ }^{16}$ The notation $M_{\mu_{1} \mu_{2} \cdots\left(\mu_{i} \cdots \mu_{j-1}\left|\mu_{j} \cdots \mu_{k}\right| \mu_{k+1} \cdots \mu_{l}\right) \cdots \mu_{n}}$ indicates we symmetrize over the indices $\mu_{i}, \cdots, \mu_{j-1}, \mu_{k+1}, \cdots, \mu_{l}$, but not over the indices $\mu_{j}, \ldots \mu_{k}$ enclosed between the bars.
} 
corresponding to vectors $d_{\mu}^{[m n]}$ and internal scalars $\zeta^{(m n)}$ in the former case and $\overline{\mathcal{Q}} r \rightarrow \beta^{ \pm}+\Omega^{+}$in the latter. The left-handed supercharge yields

$$
\begin{aligned}
{\left[\eta_{I}^{a} \mathcal{Q}_{a}^{\left(-\frac{1}{2}\right), I}, V_{r}^{\left(-\frac{1}{2}\right)}\right] } & =V_{d}^{(-1)}\left(d_{\mu}^{[m n]}=\frac{\sqrt{\alpha^{\prime}}}{2} \eta_{I}^{a}\left[k_{\mu} \delta_{a}^{b}+\left(\sigma_{\mu} \not k\right)_{a}^{b}\right] r_{b, J}^{[m}\left(\gamma^{n]} C\right)^{I J}\right) \\
& +V_{\zeta}^{(-1)}\left(\zeta^{(m n)}=\frac{1}{\sqrt{2}} \eta_{I}^{a} r_{a, J}^{(m}\left(\gamma^{n)} C\right)^{I J}\right) \\
{\left[\bar{\eta}_{\dot{a}}^{\bar{I}} \overline{\mathcal{Q}}_{\bar{I}}^{\left(-\frac{1}{2}\right), \dot{a}}, V_{r}^{\left(-\frac{1}{2}\right)}\right] } & =V_{\beta^{+}}^{(-1)}\left(\beta_{\mu}^{+, m}=\frac{1}{\sqrt{2}} \bar{\eta}_{\dot{a}}^{\bar{I}}\left(\bar{\sigma}_{\mu}^{\dot{a} a}+\alpha^{\prime} k_{\mu} k^{\dot{a} a}\right) r_{a, I}^{m} C_{\bar{I}}^{I}\right) \\
& +V_{\Omega_{+}}^{(-1)}\left(\Omega_{+}^{m n l}=-\frac{\sqrt{\alpha^{\prime}}}{4} \bar{\eta}_{\dot{a}}^{\bar{I}} \not k^{\dot{a} a} r_{a, I}^{[m}\left(\bar{\gamma}^{n l]} C\right)_{\bar{I}}^{I}\right),
\end{aligned}
$$

and the right-handed counterpart reads

$$
\begin{aligned}
{\left[\eta_{I}^{a} \mathcal{Q}_{a}^{\left(-\frac{1}{2}\right), I}, V_{\bar{r}}^{\left(-\frac{1}{2}\right)}\right] } & =V_{\beta^{-}}^{(-1)}\left(\beta_{\mu}^{-, m}=\frac{1}{\sqrt{2}} \eta_{I}^{a}\left(\sigma_{\mu a \dot{a}}+\alpha^{\prime} k_{\mu} k_{a \dot{a}}\right) \bar{r}_{\bar{I}}^{m, \dot{a}} C_{I}^{\bar{I}}\right) \\
& +V_{\Omega_{-}}^{(-1)}\left(\Omega_{-}^{m n l}=-\frac{\sqrt{\alpha^{\prime}}}{4} \eta_{I}^{a} \not_{a \dot{a}} \bar{r}^{[m|, \dot{a}, \bar{I}|}\left(\gamma^{n l]} C\right)_{\bar{I}}^{I}\right), \\
{\left[\bar{\eta}_{\dot{a}}^{\bar{I}} \overline{\mathcal{Q}}_{\bar{I}}^{\left(-\frac{1}{2}\right), \dot{a}}, V_{\bar{r}}^{\left(-\frac{1}{2}\right)}\right] } & =V_{d}^{(-1)}\left(d_{\mu}^{[m n]}=\frac{\sqrt{\alpha^{\prime}}}{2} \bar{\eta}_{\dot{a}}^{\bar{I}}\left[k_{\mu} \delta_{\dot{b}}^{\dot{a}}+\left(\bar{\sigma}_{\mu} \not k\right)^{\dot{a}}{ }_{\dot{b}}\right] \bar{r}^{[m|, \dot{b}, \bar{J}|}\left(\bar{\gamma}^{n]} C\right)_{\bar{I} \bar{J}}\right) \\
& +V_{\zeta}^{(-1)}\left(\zeta^{(m n)}=\frac{1}{\sqrt{2}} \bar{\eta}_{\dot{a}}^{\bar{I}} \bar{r}^{(m|, \dot{a}, \bar{J}|}\left(\bar{\gamma}^{n)} C\right)_{\bar{I} \bar{J}}\right) .
\end{aligned}
$$

This completes the list of SUSY transformations within the $\mathcal{N}=4$ multiplet. We will revisit these results from the spinor helicity viewpoint in section 7 .

\section{Massive supermultiplets for $\mathcal{N}=1$ SUSY}

This section is devoted to the universal SUSY multiplets common to all $D=4$ superstring compactifications which preserve $\mathcal{N}=1$ spacetime SUSY. It was already observed in [1] that 24 universal states exist, and the reference also investigates their three- and four-point couplings to massless states. We will show that they gather in three multiplets: one spin two representation of $8+8$ states and two spin $1 / 2$ representations of $2+2$ states each. The first subsections review the construction of these states and the third one contains their SUSY variations.

\subsection{NS sector}

By assembling $h=3 / 2$ combinations of the conformal fields of figure 2, one arrives at the following general form of an NS state at mass $m^{2}=1 / \alpha^{\prime}$ :

$$
V^{(-1)}=\left(\alpha_{\mu \nu} i \partial X^{\mu} \psi^{\nu}+e_{\mu \nu \lambda} \psi^{\mu} \psi^{\nu} \psi^{\lambda}+h_{\mu} \partial \psi^{\mu}+\xi_{\mu} \psi^{\mu} \mathcal{J}\right.
$$




$$
\left.+\Omega_{+} \mathcal{O}^{+}+\Omega_{-} \mathcal{O}^{-}+c_{+} G_{\text {int }}^{+}+c_{-} G_{\text {int }}^{-}\right) \mathrm{e}^{-\phi} \mathrm{e}^{i k \cdot X} .
$$

This is BRST invariant if the polarization tensors satisfy

$$
\begin{array}{lll}
0=\alpha_{\mu}{ }^{\mu}+k^{\mu} h_{\mu}+\frac{3}{2 \sqrt{\alpha^{\prime}}}\left(c_{+}+c_{-}\right) & 0=\alpha_{[\mu \nu]}+3 e_{\mu \nu \lambda} k^{\lambda} \\
0=k^{\mu} \xi_{\mu}+\frac{\sqrt{3}}{2 \sqrt{\alpha^{\prime}}}\left(c_{-}-c_{+}\right) & 0=2 \alpha^{\prime} \alpha_{\mu \nu} k^{\nu}+h_{\mu}
\end{array}
$$

Twelve physical states solve this system of equations:

- one transverse and traceless spin two tensor

$$
V_{\alpha}^{(-1)}=\frac{1}{\sqrt{2 \alpha^{\prime}}} \alpha_{\mu \nu} i \partial X^{\mu} \psi^{\nu} \mathrm{e}^{-\phi} \mathrm{e}^{i k \cdot X}, \quad k^{\mu} \alpha_{\mu \nu}=\alpha_{[\mu \nu]}=\alpha_{\mu}{ }^{\mu}=0
$$

- one transverse vector

$$
V_{d}^{(-1)}=d_{\mu} \psi^{\mu} \mathcal{J} \mathrm{e}^{-\phi} \mathrm{e}^{i k \cdot X}, \quad k^{\mu} d_{\mu}=0
$$

- two complex scalars

$$
\begin{aligned}
V_{\Phi^{ \pm}}^{(-1)}= & \frac{\Phi^{ \pm}}{2 \sqrt{2 \alpha^{\prime}}}\left[\left(\eta_{\mu \nu}+2 \alpha^{\prime} k_{\mu} k_{\nu}\right) i \partial X^{\mu} \psi^{\nu}+2 \alpha^{\prime} k_{\mu} \partial \psi^{\mu}\right. \\
& \left. \pm \frac{i \alpha^{\prime}}{3} \varepsilon_{\mu \nu \lambda \rho} \psi^{\mu} \psi^{\nu} \psi^{\lambda} k^{\rho}\right] \mathrm{e}^{-\phi} \mathrm{e}^{i k \cdot X} \\
V_{\Omega^{ \pm}}^{(-1)}= & \Omega^{ \pm} \mathcal{O}^{ \pm} \mathrm{e}^{-\phi} \mathrm{e}^{i k \cdot X} .
\end{aligned}
$$

In addition, we have spurious solutions to the BRST constraints:

$$
\begin{aligned}
& V_{\pi(\mathrm{sp})}^{(-1)} \sim {\left[\left(\pi_{\mu} k_{\nu}+k_{\mu} \pi_{\nu}\right) i \partial X^{\mu} \psi^{\nu}+2 \pi_{\mu} \partial \psi^{\mu}\right] \mathrm{e}^{-\phi} \mathrm{e}^{i k \cdot X}, \quad k^{\mu} \pi_{\mu}=0 } \\
& V_{\Sigma(\mathrm{sp})}^{(-1)} \sim\left[2 \Sigma_{[\mu \nu]} i \partial X^{\mu} \psi^{\nu}+2 \alpha^{\prime} \Sigma_{[\mu \nu} k_{\lambda]} \psi^{\mu} \psi^{\nu} \psi^{\lambda}\right] \mathrm{e}^{-\phi} \mathrm{e}^{i k \cdot X}, \quad k^{\mu} \Sigma_{\mu \nu}=0 \\
& V_{\Lambda_{0}(\mathrm{sp})}^{(-1)} \sim \Lambda_{0}\left[\left(G_{\text {int }}^{+}-G_{\text {int }}^{-}\right)-\sqrt{3 \alpha^{\prime}} k_{\mu} \psi^{\mu} \mathcal{J}\right] \mathrm{e}^{-\phi} \mathrm{e}^{i k \cdot X} \\
& V_{\Lambda_{1}(\mathrm{sp})}^{(-1)} \sim \Lambda_{1}\left[\left(\eta_{\mu \nu}+4 \alpha^{\prime} k_{\mu} k_{\nu}\right) i \partial X^{\mu} \psi^{\nu}+6 \alpha^{\prime} k_{\mu} \partial \psi^{\mu}\right. \\
&\left.+2 \sqrt{\alpha^{\prime}}\left(G_{\text {int }}^{+}+G_{\text {int }}^{-}\right)\right] \mathrm{e}^{-\phi} \mathrm{e}^{i k \cdot X}
\end{aligned}
$$

The last two spurious states allow to gauge away both the $c^{ \pm}$scalars and the longitudinal component of the massive vector $\xi_{\mu} \sim k_{\mu}$. 


\section{$5.2 \quad \mathrm{R}$ sector}

For $D=4$ fermions at mass $m^{2}=1 / \alpha^{\prime}$, the most general vertex operators built from $\mathcal{N}=1$ internal SCFT fields reads

$$
\begin{aligned}
V^{\left(-\frac{1}{2}\right)}= & \left(v_{\mu}^{a} i \partial X^{\mu} S_{a} \Sigma^{+}+\bar{\rho}_{\dot{b}}^{\mu} S_{\mu}^{\dot{b}} \Sigma^{+}+u^{a} \partial S_{a} \Sigma^{+}\right. \\
& \left.+y^{a} S_{a} \partial \Sigma^{+}+\bar{\omega}_{\dot{b}} S^{\dot{b}} \tilde{\Sigma}^{+}\right) \mathrm{e}^{-\phi / 2} \mathrm{e}^{i k \cdot X}
\end{aligned}
$$

see figure 2. Invariance under $Q_{1}$ yields three independent BRST constraints:

$$
\begin{aligned}
& 0=2 \alpha^{\prime} v^{\mu, a} \not k_{a \dot{b}}+\sqrt{2} \bar{\rho}_{\dot{b}}^{\mu}+\frac{1}{2} u^{a} \sigma_{a \dot{b}}^{\mu}, \\
& 0=k_{\mu} \bar{\rho}_{\dot{b}}^{\mu}+\frac{1}{2} \sqrt{\frac{3}{2 \alpha^{\prime}}} \bar{\omega}_{\dot{b}}, \\
& 0=y^{a}+2 \sqrt{\frac{\alpha^{\prime}}{3} \bar{\omega}_{\dot{b}} k^{\dot{b} a} .} .
\end{aligned}
$$

They allow to express any wavefunction in terms of $v_{\mu}^{a}$

$$
\begin{aligned}
u^{a} & =\alpha^{\prime} v_{\mu}^{b}\left(\not k \bar{\sigma}^{\mu}\right)_{b}{ }^{a} \\
\bar{\rho}_{\mu \dot{b}} & =-\sqrt{2} \alpha^{\prime}\left(v_{\mu}^{a} \not k_{a \dot{b}}+\frac{1}{4} v_{\lambda}^{a}\left(\not k \bar{\sigma}^{\lambda} \sigma_{\mu}\right)_{a \dot{b}}\right) \\
\bar{\omega}_{\dot{b}} & =\sqrt{\frac{\alpha^{\prime}}{3}}\left(2 \alpha^{\prime} k^{\mu} v_{\mu}^{a} \not k_{a \dot{b}}-v_{\mu}^{a} \sigma_{a \dot{b}}^{\mu}\right) \\
y^{a} & =\frac{2 \alpha^{\prime}}{3}\left(v_{\mu}^{b}\left(\sigma^{\mu} \not\right)_{b}{ }^{a}-2 k^{\mu} v_{\mu}^{a}\right) .
\end{aligned}
$$

The same set of states exists with opposite $S O(1,3)$ chirality and internal $U(1)$ charge. Including them, we have four physical solutions to (5.13) and four solutions to the conjugate system of equations:

- two transverse and $\sigma$ traceless spin 3/2 vector spinors

$$
\begin{aligned}
V_{\chi}^{\left(-\frac{1}{2}\right)} & =\frac{1}{\sqrt{2} \alpha^{\prime 1 / 4}} \chi_{\mu}^{a}\left(i \partial X^{\mu} S_{a}-\sqrt{2} \alpha^{\prime} \not k_{a \dot{b}} S^{\mu \dot{b}}\right) \Sigma^{+} \mathrm{e}^{-\phi / 2} \mathrm{e}^{i k \cdot X}, \\
V_{\bar{\chi}}^{\left(-\frac{1}{2}\right)} & =\frac{1}{\sqrt{2} \alpha^{\prime / 4}} \bar{\chi}_{\dot{a}}^{\mu}\left(i \partial X_{\mu} S^{\dot{a}}-\sqrt{2} \alpha^{\prime} \not k^{\dot{a} b} S_{\mu b}\right) \Sigma^{-} \mathrm{e}^{-\phi / 2} \mathrm{e}^{i k \cdot X}, \\
0 & =k^{\mu} \chi_{\mu}^{a}=\chi_{\mu}^{a} \sigma_{a \dot{b}}^{\mu}=k_{\mu} \bar{\chi}_{\dot{a}}^{\mu}=\bar{\chi}_{\dot{a}}^{\mu} \bar{\sigma}_{\mu}^{\dot{a} b}
\end{aligned}
$$

- two spin $1 / 2$ fermions

$$
\begin{aligned}
V_{a}^{\left(-\frac{1}{2}\right)} & =\frac{\alpha^{\prime 1 / 4}}{2} a^{b}\left(\left(\sigma_{\mu} \not\right)_{b}{ }^{a} S_{a} i \partial X^{\mu}-4 \partial S_{b}\right) \Sigma^{+} \mathrm{e}^{-\phi / 2} \mathrm{e}^{i k \cdot X}, \\
V_{\bar{a}}^{\left(-\frac{1}{2}\right)} & =\frac{\alpha^{\prime 1 / 4}}{2} \bar{a}_{\dot{b}}\left(\left(\bar{\sigma}_{\mu} \not k\right)^{\dot{b}}{ }_{\dot{a}} S^{\dot{a}} i \partial X^{\mu}-4 \partial S^{\dot{b}}\right) \Sigma^{-} \mathrm{e}^{-\phi / 2} \mathrm{e}^{i k \cdot X} .
\end{aligned}
$$


Spurious solutions can gauge away the internal excitations with wavefunctions $y^{a}$ and $\bar{\omega}_{\dot{b}}$ :

$$
\begin{aligned}
V_{\Theta(\mathrm{sp})}^{\left(-\frac{1}{2}\right)} \sim \Theta^{a}\left[\left(k_{a \dot{a}} \bar{\sigma}_{\mu}^{\dot{a} b}+4 k_{\mu} \delta_{a}^{b}\right) i \partial X^{\mu} S_{b} \Sigma^{+}-2 \sqrt{2}\left(\alpha^{\prime} k^{\mu} \not k_{a \dot{b}}+\frac{1}{4} \sigma_{a \dot{b}}^{\mu}\right) S_{\mu}^{\dot{b}} \Sigma^{+}\right. \\
\left.+6 \partial S_{a} \Sigma^{+}+4 S_{a} \partial \Sigma^{+}-2 \sqrt{3 \alpha^{\prime}} \not k_{a \dot{b}} S^{\dot{b}} \tilde{\Sigma}^{+}\right] \mathrm{e}^{-\phi / 2} \mathrm{e}^{i k \cdot X}, \\
V_{\bar{\Theta}(\mathrm{sp})}^{\left(-\frac{1}{2}\right)} \sim \bar{\Theta}_{\dot{b}}\left[\left(\not k^{\dot{b} a} \sigma_{a \dot{a}}^{\mu}+4 k^{\mu} \delta_{\dot{a}}^{\dot{b}}\right) i \partial X_{\mu} S^{\dot{a}} \Sigma^{-}-2 \sqrt{2}\left(\alpha^{\prime} k_{\mu} \not k^{\dot{b} a}+\frac{1}{4} \bar{\sigma}_{\mu}^{\dot{b} a}\right) S_{a}^{\mu} \Sigma^{-}\right. \\
\left.+6 \partial S^{\dot{b}} \Sigma^{-}+4 S^{\dot{b}} \partial \Sigma^{-}-2 \sqrt{3 \alpha^{\prime}} \not k^{\dot{b} a} S_{a} \tilde{\Sigma}^{-}\right] \mathrm{e}^{-\phi / 2} \mathrm{e}^{i k \cdot X} .
\end{aligned}
$$

\subsection{SUSY transformations}

The notation for the $\mathcal{N}=1$ multiplets can be kept lighter because of the Abelian R-symmetry group $U(1)$. The supercharge operators do not carry any R-symmetry indices, only an Abelian charge of $\pm \sqrt{3} / 2$. After performing SUSY variation on all the bosonic and fermionic states in $\mathcal{N}=1$ SUSY, we find that these states split into three separate massive supermultiplets - a spin two multiplet $\{\alpha, \chi, \bar{\chi}, d\}$, two spin- $\frac{1}{2}$ multiplets $\left\{\Phi^{+}, \bar{a}, \Omega^{-}\right\}$and $\left\{\Omega^{+}, a, \Phi^{-}\right\}$, see figure 6 below. We will show our results of the SUSY transformations in order.

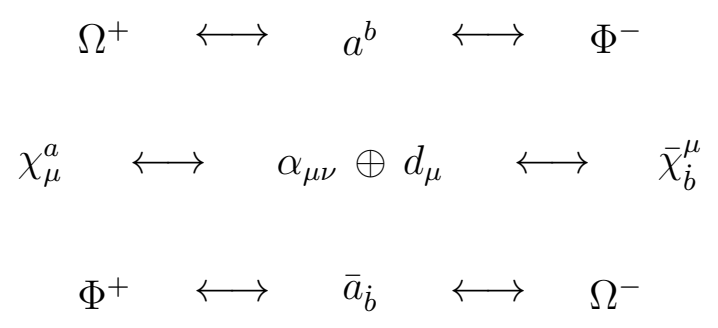

Figure 6: The three disconnected $\mathcal{N}=1$ SUSY multiplets at the first mass level: As before, $\mathcal{Q}_{a}$ $\left(\overline{\mathcal{Q}}^{\dot{b}}\right)$ action takes states along a left (right) arrow.

\subsubsection{SUSY variation of the spin two supermultiplet}

The spin two multiplet includes a spin two boson $\alpha_{\mu \nu}$, a vector $d_{\mu}$, and two spin- $\frac{3}{2}$ fermions $\chi_{\mu}^{a}$, $\bar{\chi}_{\mu, \dot{a}}$ with opposite chirality. The SUSY transformation of the bosonic states are:

$$
\begin{aligned}
{\left[\eta^{a} \mathcal{Q}_{a}^{\left(+\frac{1}{2}\right)}, V_{\alpha}^{(-1)}\right] } & =V_{\chi}^{\left(-\frac{1}{2}\right)}\left(\chi_{\mu}^{b}=\frac{1}{\sqrt{2}} \eta^{a} \alpha_{\mu \nu}\left(\not k \bar{\sigma}^{\nu}\right)_{a}^{b}\right), \\
{\left[\bar{\eta}_{\dot{a}} \overline{\mathcal{Q}}^{\left(+\frac{1}{2}\right), \dot{a}}, V_{\alpha}^{(-1)}\right] } & =V_{\bar{\chi}}^{\left(-\frac{1}{2}\right)}\left(\bar{\chi}_{\mu, \dot{b}}=\frac{1}{\sqrt{2}} \bar{\eta}_{\dot{a}} \alpha_{\mu \nu}\left(\not k \sigma^{\nu}\right)^{\dot{a}} \dot{b}\right), \\
{\left[\eta^{a} \mathcal{Q}_{a}^{\left(+\frac{1}{2}\right)}, V_{d}^{(-1)}\right] } & =V_{\chi}^{\left(-\frac{1}{2}\right)}\left(\chi_{\mu}^{b}=\frac{-1}{2 \sqrt{3 \alpha^{\prime}}} \eta^{a}\left[3 d_{\mu} \delta_{a}^{b}+\left(\phi \bar{\sigma}_{\mu}+\alpha^{\prime} k_{\mu} \phi \lambda\right)_{a}{ }^{b}\right]\right),
\end{aligned}
$$




$$
\left[\bar{\eta}_{\dot{a}} \overline{\mathcal{Q}}^{\left(+\frac{1}{2}\right), \dot{a}}, V_{d}^{(-1)}\right]=V_{\bar{\chi}}^{\left(-\frac{1}{2}\right)}\left(\bar{\chi}_{\mu, \dot{b}}=\frac{1}{2 \sqrt{3 \alpha^{\prime}}} \bar{\eta}_{\dot{a}}\left[3 d_{\mu} \delta^{\dot{a}}{ }_{\dot{b}}+\left(\not d \sigma_{\mu}+\alpha^{\prime} k_{\mu} \not k k\right)^{\dot{a}}{ }_{\dot{b}}\right]\right) .
$$

The SUSY transformation of the fermionic states are:

$$
\begin{aligned}
{\left[\eta^{a} \mathcal{Q}_{a}^{\left(-\frac{1}{2}\right)}, V_{\chi}^{\left(-\frac{1}{2}\right)}\right] } & =0, \\
{\left[\bar{\eta}_{\dot{a}} \overline{\mathcal{Q}}^{\left(-\frac{1}{2}\right), \dot{a}}, V_{\chi}^{\left(-\frac{1}{2}\right)}\right] } & =V_{\alpha}^{(-1)}\left(\alpha_{\mu \nu}=\frac{1}{\sqrt{2}} \bar{\eta}_{\dot{a}}\left(\bar{\sigma}_{(\mu}^{\dot{a} a} \chi_{\nu) a}+\alpha^{\prime} k^{\dot{a} a} k_{(\mu} \chi_{\nu), a}\right)\right) \\
& +V_{d}^{(-1)}\left(d_{\mu}=\frac{\sqrt{3 \alpha^{\prime}}}{2} \bar{\eta}_{\dot{a}} k^{\dot{a} a} \chi_{\mu, a}\right), \\
{\left[\eta^{a} \mathcal{Q}_{a}^{\left(-\frac{1}{2}\right)}, V_{\bar{\chi}}^{\left(-\frac{1}{2}\right)}\right] } & =V_{\alpha}^{(-1)}\left(\alpha_{\mu \nu}=\frac{1}{\sqrt{2}} \eta^{a}\left(\sigma_{(\mu|a \dot{a}|} \bar{\chi}_{\nu)}^{\dot{a}}+\alpha^{\prime} k_{a \dot{a}} k_{(\mu} \bar{\chi}_{\nu)}^{\dot{a}}\right)\right) \\
& +V_{d}^{(-1)}\left(d_{\mu}=-\frac{\sqrt{3 \alpha^{\prime}}}{2} \eta^{a} \nvdash_{a \dot{a}} \bar{\chi}_{\mu}^{\dot{a}}\right), \\
{\left[\bar{\eta}_{\dot{a}} \overline{\mathcal{Q}}^{\left(-\frac{1}{2}\right), \dot{a}}, V_{\bar{\chi}}^{\left(-\frac{1}{2}\right)}\right] } & =0 .
\end{aligned}
$$

Note that the signs of the SUSY transformations between spin- $\frac{3}{2}$ and spin one are sensitive to the chirality, see the relative signs between (5.23) and (5.24) as well as (5.26) and (5.27). This is necessary for consistent closure of the SUSY algebra and can be neatly represented by a chirality matrix $\gamma^{5}$ when passing to Dirac spinor notation.

\subsubsection{SUSY variation of the spin $1 / 2$ supermultiplets}

The first spin- $\frac{1}{2}$ multiplet $\left\{\Phi^{+}, \bar{a}, \Omega^{-}\right\}$includes a right-handed spin- $\frac{1}{2}$ fermion $\bar{a}$ and two scalars $\Phi^{+}, \Omega^{-}$. It is governed by the following SUSY transformations:

$$
\begin{aligned}
{\left[\eta^{b} \mathcal{Q}_{b}^{\left(+\frac{1}{2}\right)}, V_{\Phi^{+}}^{(-1)}\right] } & =0, \\
{\left[\bar{\eta}_{\dot{b}} \overline{\mathcal{Q}}^{\left(+\frac{1}{2}\right), \dot{b}}, V_{\Phi^{+}}^{(-1)}\right] } & =V_{\bar{a}}^{\left(-\frac{1}{2}\right)}\left(\bar{a}_{\dot{b}}=-\alpha^{\prime-\frac{1}{2}} \Phi^{+} \bar{\eta}_{\dot{b}}\right), \\
{\left[\eta^{b} \mathcal{Q}_{b}^{\left(+\frac{1}{2}\right)}, V_{\Omega^{-}}^{(-1)}\right] } & =V_{\bar{a}}^{\left(-\frac{1}{2}\right)}\left(\bar{a}_{\dot{b}}=\Omega^{-} \eta^{b} k_{b \dot{b}}\right), \\
{\left[\bar{\eta}_{\dot{b}} \overline{\mathcal{Q}}^{\left(+\frac{1}{2}\right), \dot{b}}, V_{\Omega^{-}}^{(-1)}\right] } & =0,
\end{aligned}
$$

and

$$
\begin{aligned}
{\left[\eta^{b} \mathcal{Q}_{b}^{\left(-\frac{1}{2}\right)}, V_{\bar{a}}^{\left(-\frac{1}{2}\right)}\right] } & =V_{\Phi^{+}}^{(-1)}\left(\Phi^{+}=\sqrt{\alpha^{\prime}} \eta^{b} k_{b \dot{b}} \bar{a}^{\dot{b}}\right), \\
{\left[\bar{\eta}_{\dot{b}} \overline{\mathcal{Q}}^{\left(-\frac{1}{2}\right), \dot{b}}, V_{\bar{a}}^{\left(-\frac{1}{2}\right)}\right] } & =V_{\Omega^{-}}^{(-1)}\left(\Omega^{-}=\bar{\eta}_{\dot{b}} \bar{a}^{\dot{b}}\right) .
\end{aligned}
$$

For $\left\{\Omega^{+}, a, \Phi^{-}\right\}$multiplet of opposite R-symmetry charges and fermion chirality, we obtain

$$
\left[\eta^{b} \mathcal{Q}_{b}^{\left(+\frac{1}{2}\right)}, V_{\Phi^{-}}^{(-1)}\right]=V_{a}^{\left(-\frac{1}{2}\right)}\left(a^{b}=-\alpha^{-\frac{1}{2}} \Phi^{-} \eta^{b}\right),
$$




$$
\begin{aligned}
{\left[\bar{\eta}_{\dot{b}} \overline{\mathcal{Q}}^{\left(+\frac{1}{2}\right), \dot{b}}, V_{\Phi^{-}}^{(-1)}\right] } & =0, \\
{\left[\eta^{b} \mathcal{Q}_{b}^{\left(+\frac{1}{2}\right)}, V_{\Omega^{+}}^{(-1)}\right] } & =0, \\
{\left[\bar{\eta}_{\dot{b}} \overline{\mathcal{Q}}^{\left(+\frac{1}{2}\right), \dot{b}}, V_{\Omega^{+}}^{(-1)}\right] } & =V_{a}^{\left(-\frac{1}{2}\right)}\left(a^{b}=\Omega^{+} \bar{\eta}_{\dot{b}} k^{\dot{b} b}\right),
\end{aligned}
$$

and

$$
\begin{aligned}
{\left[\eta^{b} \mathcal{Q}_{b}^{\left(-\frac{1}{2}\right)}, V_{a}^{\left(-\frac{1}{2}\right)}\right] } & =V_{\Omega^{+}}^{(-1)}\left(\Omega^{+}=\eta^{b} a_{b}\right), \\
{\left[\bar{\eta}_{\dot{b}} \overline{\mathcal{Q}}^{\left(-\frac{1}{2}\right), \dot{b}}, V_{a}^{\left(-\frac{1}{2}\right)}\right] } & =V_{\Phi^{-}}^{(-1)}\left(\Phi^{-}=\sqrt{\alpha^{\prime}} \bar{\eta}_{\dot{b}} k^{\dot{b} b} a_{b}\right) .
\end{aligned}
$$

We will explore the helicity structure of these results in section 7 .

\section{Massive supermultiplets for $\mathcal{N}=2$ SUSY}

In this section, we will show that the first mass level in compactifications with $\mathcal{N}=2$ spacetime SUSY is populated by 80 universal states which are aligned into one $24+24$ state multiplet of highest spin two and two $8+8$ state multiplets of maximum spin one.

\subsection{NS sector}

According to the CFT operator content shown in figure 3, we make the following general ansatz for an NS state at the first mass level:17

$$
\begin{aligned}
V^{(-1)}= & \left(\alpha_{\mu \nu} i \partial X^{\mu} \psi^{\nu}+e_{\mu \nu \lambda} \psi^{\mu} \psi^{\nu} \psi^{\lambda}+h_{\mu} \partial \psi^{\mu}+Y_{+} \partial \mathrm{e}^{i H}+Y_{-} \partial \mathrm{e}^{-i H}\right. \\
& +\beta_{\mu}^{+} i \partial X^{\mu} \mathrm{e}^{i H}+\beta_{\mu}^{-} i \partial X^{\mu} \mathrm{e}^{-i H}+\gamma_{\mu}^{+} \psi^{\mu} i \partial Z^{+}+\gamma_{\mu}^{-} \psi^{\mu} i \partial Z^{-} \\
& +\xi_{\mu} \psi^{\mu} i \partial H+d_{\mu}^{A} \mathcal{J}_{A} \psi^{\mu}+\Omega_{+}^{A} \mathcal{J}_{A} \mathrm{e}^{i H}+\Omega_{-}^{A} \mathcal{J}_{A} \mathrm{e}^{-i H} \\
& +\zeta_{++} i \partial Z^{+} \mathrm{e}^{i H}+\zeta_{--} i \partial Z^{-} \mathrm{e}^{-i H}+\zeta_{-+} i \partial Z^{-} \mathrm{e}^{i H}+\zeta_{+-} i \partial Z^{+} \mathrm{e}^{-i H} \\
& \left.+\omega_{\mu \nu}^{+} \psi^{\mu} \psi^{\nu} \mathrm{e}^{i H}+\omega_{\mu \nu}^{-} \psi^{\mu} \psi^{\nu} \mathrm{e}^{-i H}+c_{i}{ }^{j} \lambda^{i} g_{j}\right) \mathrm{e}^{-\phi} \mathrm{e}^{i k \cdot X}
\end{aligned}
$$

Requiring BRST invariance under $Q_{1}$ yields the following on-shell conditions:

$$
\begin{array}{rlrl}
0 & =\alpha_{\mu}{ }^{\mu}+k^{\mu} h_{\mu}+\zeta_{+-}+\zeta_{-+}-\alpha^{\prime-1 / 2} c_{i}{ }^{i} & \\
0 & =2 \alpha^{\prime} \alpha_{\mu \nu} k^{\nu}+h_{\mu} & 0 & =k^{\mu} d_{\mu}^{A}+\frac{1}{\sqrt{2 \alpha^{\prime}}}\left(\tau^{A}\right)^{i}{ }_{j} c_{i}{ }^{j} \\
0 & =\alpha_{[\mu \nu]}+3 e_{\mu \nu \lambda} k^{\lambda} & 0 & =Y_{ \pm}+2 \alpha^{\prime} \gamma_{\mu}^{ \pm} k^{\mu} \\
0 & =\beta_{\mu}^{ \pm}-\gamma_{\mu}^{ \pm}+2 k^{\nu} \omega_{\nu \mu}^{ \pm} & 0 & =k^{\mu} \xi_{\mu}+\zeta_{-+}-\zeta_{+-}
\end{array}
$$

\footnotetext{
${ }^{17}$ Recall that we have non-Abelian R-symmetry $S U(2)$ in this setting, and $i, j=1,2$ denote its spinor indices whereas $A=1,2,3$ are adjoint indices.
} 
These BRST constraints admit 40 physical solutions:

- one transverse and traceless spin two tensor

$$
V_{\alpha}^{(-1)}=\frac{1}{\sqrt{2 \alpha^{\prime}}} \alpha_{\mu \nu} i \partial X^{\mu} \psi^{\nu} \mathrm{e}^{-\phi} \mathrm{e}^{i k \cdot X}, \quad k^{\mu} \alpha_{\mu \nu}=\alpha_{[\mu \nu]}=\alpha_{\mu}{ }^{\mu}=0
$$

- eight transverse vectors three of which form an R-symmetry triplet (note the sign difference in the pseudovector parts of $\beta^{ \pm}$and $\omega^{ \pm}$)

$$
\begin{aligned}
& V_{\xi}^{(-1)}= \xi_{\mu} \psi^{\mu} i \partial H \mathrm{e}^{-\phi} \mathrm{e}^{i k \cdot X}, \quad k^{\mu} \xi_{\mu}=0 \\
& V_{d}^{(-1)}= d_{\mu}^{A} \psi^{\mu} \mathcal{J}_{A} \mathrm{e}^{-\phi} \mathrm{e}^{i k \cdot X}, \quad k^{\mu} d_{\mu}^{A}=0 \\
& V_{\beta^{ \pm}}^{(-1)}= \frac{1}{2 \sqrt{2 \alpha^{\prime}}} \beta_{\mu}^{ \pm}\left(i \partial X^{\mu} \mathrm{e}^{ \pm i H}+i \partial Z^{ \pm} \psi^{\mu}\right. \\
&\left. \pm i \alpha^{\prime} \varepsilon^{\mu \nu \lambda \rho} k_{\nu} \psi_{\lambda} \psi_{\rho} \mathrm{e}^{ \pm i H}\right) \mathrm{e}^{-\phi} \mathrm{e}^{i k \cdot X}, \quad k^{\mu} \beta_{\mu}^{ \pm}=0 \\
& V_{\omega^{ \pm}}^{(-1)}=\frac{1}{2 \sqrt{2 \alpha^{\prime}}} \omega_{\mu}^{ \pm}\left(i \partial X^{\mu} \mathrm{e}^{ \pm i H}+i \partial Z^{ \pm} \psi^{\mu}\right. \\
&\left.\mp i \alpha^{\prime} \varepsilon^{\mu \nu \lambda \rho} k_{\nu} \psi_{\lambda} \psi_{\rho} \mathrm{e}^{ \pm i H}\right) \mathrm{e}^{-\phi} \mathrm{e}^{i k \cdot X}, \quad k^{\mu} \omega_{\mu}^{ \pm}=0
\end{aligned}
$$

- eleven real scalar degrees of freedom

$$
\begin{aligned}
V_{\Phi^{ \pm}}^{(-1)}= & \frac{\Phi^{ \pm}}{2 \sqrt{2 \alpha^{\prime}}}\left[\left(\eta_{\mu \nu}+2 \alpha^{\prime} k_{\mu} k_{\nu}\right) i \partial X^{\mu} \psi^{\nu}+2 \alpha^{\prime} k_{\mu} \partial \psi^{\mu}\right. \\
& \left. \pm \frac{i \alpha^{\prime}}{3} \varepsilon_{\mu \nu \lambda \rho} \psi^{\mu} \psi^{\nu} \psi^{\lambda} k^{\rho}\right] \mathrm{e}^{-\phi} \mathrm{e}^{i k \cdot X} \\
V_{\phi}^{(-1)}= & \frac{1}{\sqrt{6 \alpha^{\prime}}} \phi\left[i \partial Z^{+} \mathrm{e}^{-i H}+i \partial Z^{-} \mathrm{e}^{i H}+\sqrt{\alpha^{\prime}} G_{i}^{i}\right] \mathrm{e}^{-\phi} \mathrm{e}^{i k \cdot X} \\
V_{\Omega^{ \pm}}^{(-1)}= & \Omega_{A}^{ \pm} \mathrm{e}^{ \pm i H} \mathcal{J}^{A} \mathrm{e}^{-\phi} \mathrm{e}^{i k \cdot X} \\
V_{\zeta^{ \pm}}^{(-1)}= & \frac{1}{\sqrt{2 \alpha^{\prime}}} \zeta^{ \pm} i \partial Z^{ \pm} \mathrm{e}^{ \pm i H} \mathrm{e}^{-\phi} \mathrm{e}^{i k \cdot X} .
\end{aligned}
$$

In addition, we have numerous spurious states:

$$
\begin{aligned}
& V_{\pi(\mathrm{sp})}^{(-1)} \sim\left[\left(\pi_{\mu} k_{\nu}+k_{\mu} \pi_{\nu}\right) i \partial X^{\mu} \psi^{\nu}+2 \pi_{\mu} \partial \psi^{\mu}\right] \mathrm{e}^{-\phi} \mathrm{e}^{i k \cdot X}, \quad k^{\mu} \pi_{\mu}=0 \\
& V_{\Sigma(\mathrm{sp})}^{(-1)} \sim\left[2 \Sigma_{[\mu \nu]} i \partial X^{\mu} \psi^{\nu}+2 \alpha^{\prime} \Sigma_{[\mu \nu} k_{\lambda]} \psi^{\mu} \psi^{\nu} \psi^{\lambda}\right] \mathrm{e}^{-\phi} \mathrm{e}^{i k \cdot X}, \quad k^{\mu} \Sigma_{\mu \nu}=0 \\
& V_{\Lambda_{0}(\mathrm{sp})}^{(-1)} \sim \Lambda_{0}^{A}\left[k_{\mu} \psi^{\mu} \mathcal{J}_{A}+\frac{1}{\sqrt{2 \alpha^{\prime}}}\left(\tau_{A}\right)^{j}{ }_{i} G^{i}{ }_{j}\right] \mathrm{e}^{-\phi} \mathrm{e}^{i k \cdot X}, \\
& V_{\Lambda_{1}(\mathrm{sp})}^{(-1)} \sim \Lambda_{1}\left[\left(\eta_{\mu \nu}+4 \alpha^{\prime} k_{\mu} k_{\nu}\right) i \partial X^{\mu} \psi^{\nu}+6 \alpha^{\prime} k_{\mu} \partial \psi^{\mu}\right. \\
&\left.+i \partial Z^{+} \mathrm{e}^{-i H}+i \partial Z^{-} \mathrm{e}^{i H}-2 \sqrt{\alpha^{\prime}} G^{i}{ }_{i}\right] \mathrm{e}^{-\phi} \mathrm{e}^{i k \cdot X}
\end{aligned}
$$




$$
\begin{aligned}
V_{\Lambda_{2}^{ \pm}(\mathrm{sp})}^{(-1)} & \sim \Lambda_{2}^{ \pm}\left[k_{\mu}\left(i \partial Z^{ \pm} \psi^{\mu}+i \partial X^{\mu} \mathrm{e}^{ \pm i H}\right)+2 \partial \mathrm{e}^{ \pm i H}\right] \mathrm{e}^{-\phi} \mathrm{e}^{i k \cdot X} \\
V_{\Lambda_{3}(\mathrm{sp})}^{(-1)} & \sim \Lambda_{3}\left[2 \alpha^{\prime} k_{\mu} \psi^{\mu} i \partial H+i \partial Z^{-} \mathrm{e}^{i H}-i \partial Z^{+} \mathrm{e}^{-i H}\right] \mathrm{e}^{-\phi} \mathrm{e}^{i k \cdot X}, \\
V_{\Lambda_{4}^{ \pm}(\mathrm{sp})}^{(-1)} & \sim \Lambda_{4 \mu}^{ \pm}\left[2 \alpha^{\prime} k_{\nu} \psi^{\nu} \psi^{\mu} \mathrm{e}^{ \pm i H}+i \partial X^{\mu} \mathrm{e}^{ \pm i H}-i \partial Z^{ \pm} \psi^{\mu}\right] \mathrm{e}^{-\phi} \mathrm{e}^{i k \cdot X}, \quad k^{\mu} \Lambda_{4 \mu}^{ \pm}=0 .
\end{aligned}
$$

They allow to eliminate the longitudinal components of six vectors and of the two-forms $\omega_{\mu \nu}^{ \pm}$. The latter therefore dualize to transverse pseudovectors entering the $\beta_{\mu}^{ \pm}$and $\omega_{\mu}^{ \pm}$states. By combining with the $\Lambda_{1}$ spurious state, one can transform the $\phi$ solution into a form without internal $c=6$ supercurrents:

$$
\begin{aligned}
V_{\phi}^{(-1)}=\frac{1}{\sqrt{6 \alpha^{\prime}}} \phi\left[\left(\eta_{\mu \nu}\right.\right. & \left.+4 \alpha^{\prime} k_{\mu} k_{\nu}\right) i \partial X^{\mu} \psi^{\nu}+6 \alpha^{\prime} k_{\mu} \partial \psi^{\mu} \\
& \left.+3\left(i \partial Z^{+} \mathrm{e}^{-i H}+i \partial Z^{-} \mathrm{e}^{i H}\right)\right] \mathrm{e}^{-\phi} \mathrm{e}^{i k \cdot X}
\end{aligned}
$$

\subsection{R sector}

In the $\mathrm{R}$ sector of the first mass level in $\mathcal{N}=2$ scenarios, the vertex operator ansatz in one chirality sector includes nine SCFT operators:

$$
\begin{aligned}
V^{\left(-\frac{1}{2}\right)}= & \left\{v_{\mu i}^{a} i \partial X^{\mu} S_{a} \lambda^{i} \mathrm{e}^{i H / 2}+\bar{\rho}_{\dot{b} i}^{\mu} S_{\mu}^{\dot{b}} \lambda^{i} \mathrm{e}^{i H / 2}+u_{i}^{a} \partial S_{a} \lambda^{i} \mathrm{e}^{i H / 2}+\bar{r}_{+\dot{b} i} i \partial Z^{+} S^{\dot{b}} \lambda^{i} \mathrm{e}^{-i H / 2}\right. \\
& +\bar{r}_{-\dot{b} i} i \partial Z^{-} S^{\dot{b}} \lambda^{i} \mathrm{e}^{-i H / 2}+\omega_{i}^{a} S_{a} \lambda^{i} \partial \mathrm{e}^{i H / 2}+y_{i}^{a} S_{a} \partial \lambda^{i} \mathrm{e}^{i H / 2} \\
& \left.+\bar{\ell}_{\dot{b} i} S^{\dot{b}} g^{i} \mathrm{e}^{i H / 2}+\psi_{i}^{a} S_{a} \lambda^{i} \mathrm{e}^{-3 i H / 2}\right\} \mathrm{e}^{-\phi / 2} \mathrm{e}^{i k \cdot X}
\end{aligned}
$$

The system of BRST constraints can be reduced to the following independent set:

$$
\begin{array}{rlrl}
0=2 k_{\mu} \bar{\rho}_{\dot{b} i}^{\mu}+\bar{r}_{+\dot{b} i}-\sqrt{\frac{1}{\alpha^{\prime}}} \bar{\ell}_{\dot{b} i} & 0 & =\omega_{i}^{a}+2 \sqrt{2} \alpha^{\prime} \bar{r}_{+\dot{b} i} \not k^{\dot{b} a} \\
0=2 \alpha^{\prime} v_{i}^{\mu, a} \not k_{a \dot{b}}+\sqrt{2} \bar{\rho}_{\dot{b} i}^{\mu}+\frac{1}{2} u_{i}^{a} \sigma_{a \dot{b}}^{\mu} & 0 & =\psi_{i}^{a}+\sqrt{2} \alpha^{\prime} \bar{r}_{-\dot{b i}} \not k^{\dot{b} a}
\end{array} .
$$

Adding a sector of opposite chirality and internal charge gives rise to 40 physical solutions. All of them transform in the fundamental representation of the $S U(2)$ R-symmetry:

- four transverse and $\sigma$ traceless spin 3/2 vector spinors

$$
\begin{aligned}
V_{\chi}^{\left(-\frac{1}{2}\right)} & =\frac{1}{\sqrt{2} \alpha^{\prime 1 / 4}} \chi_{\mu, i}^{a}\left(i \partial X^{\mu} S_{a}-\sqrt{2} \alpha^{\prime} \not k_{a \dot{b}} S^{\mu \dot{b}}\right) \lambda^{i} \mathrm{e}^{i H / 2} \mathrm{e}^{-\phi / 2} \mathrm{e}^{i k \cdot X} \\
V_{\bar{\chi}}^{\left(-\frac{1}{2}\right)} & =\frac{1}{\sqrt{2} \alpha^{\prime 1 / 4}} \bar{\chi}_{\dot{b}, i}^{\mu}\left(i \partial X_{\mu} S^{\dot{b}}-\sqrt{2} \alpha^{\prime} \not k^{\dot{b} a} S_{\mu a}\right) \lambda^{i} \mathrm{e}^{-i H / 2} \mathrm{e}^{-\phi / 2} \mathrm{e}^{i k \cdot X} \\
0 & =k^{\mu} \chi_{\mu i}^{a}=\chi_{\mu i}^{a} \sigma_{a \dot{b}}^{\mu}=k_{\mu} \bar{\chi}_{\dot{b} i}^{\mu}=\bar{\chi}_{\dot{b} i}^{\mu} \bar{\sigma}_{\mu}^{\dot{b} a}
\end{aligned}
$$


- $\operatorname{six} \operatorname{spin} 1 / 2$ fermions:

$$
\begin{aligned}
& V_{a}^{\left(-\frac{1}{2}\right)}= \frac{\alpha^{\prime 1 / 4}}{2} a_{i}^{b}\left(\left(\sigma_{\mu} \not k\right)_{b}{ }^{a} S_{a} i \partial X^{\mu}-4 \partial S_{b}\right) \lambda^{i} \mathrm{e}^{i H / 2} \mathrm{e}^{-\phi / 2} \mathrm{e}^{i k \cdot X}, \\
& V_{\bar{a}}^{\left(-\frac{1}{2}\right)}= \frac{\alpha^{\prime 1 / 4}}{2} \bar{a}_{\dot{a}, i}\left(\left(\bar{\sigma}_{\mu} \not\right)^{\dot{a}}{ }_{\dot{b}} S^{\dot{b}} i \partial X^{\mu}-4 \partial S^{\dot{a}}\right) \lambda^{i} \mathrm{e}^{-i H / 2} \mathrm{e}^{-\phi / 2} \mathrm{e}^{i k \cdot X}, \\
& V_{r}^{\left(-\frac{1}{2}\right)}= \frac{1}{\sqrt{2} \alpha^{\prime 1 / 4}} r_{i}^{a}\left(i \partial Z^{+} S_{a} \mathrm{e}^{i H / 2}-\sqrt{2} \alpha^{\prime} \not k_{a \dot{b}} S^{\dot{b}} \mathrm{e}^{3 i H / 2}\right) \lambda^{i} \mathrm{e}^{-\phi / 2} \mathrm{e}^{i k \cdot X}, \\
& V_{\bar{r}}^{\left(-\frac{1}{2}\right)}= \frac{1}{\sqrt{2} \alpha^{\prime 1 / 4}} \bar{r}_{\dot{b}, i}\left(i \partial Z^{-} S^{\dot{b}} \mathrm{e}^{-i H / 2}-\sqrt{2} \alpha^{\prime} \not k^{\dot{b} a} S_{a} \mathrm{e}^{-3 i H / 2}\right) \lambda^{i} \mathrm{e}^{-\phi / 2} \mathrm{e}^{i k \cdot X}, \\
& V_{s}^{\left(-\frac{1}{2}\right)}= \frac{1}{\sqrt{3} \alpha^{\prime 1 / 4}} s_{i}^{a}\left(i \partial Z^{-} S_{a} \lambda^{i} \mathrm{e}^{i H / 2}+\sqrt{\alpha^{\prime}} S_{a} g^{i} \mathrm{e}^{-i H / 2}\right. \\
& V_{\bar{s}}^{\left(-\frac{1}{2}\right)}= \frac{1}{\sqrt{3} \alpha^{\prime 1 / 4}} \bar{s}_{\dot{b}, i}\left(i \partial Z^{+} S^{\dot{b}} \lambda^{i} \mathrm{e}^{-i H / 2}+\sqrt{\alpha^{\prime}} S^{\dot{b}} g^{i} \mathrm{e}^{i H / 2}\left(S^{\dot{b}} \partial \lambda^{i} \mathrm{e}^{-i H / 2}-2 S^{\dot{b}} \lambda^{i} \partial \mathrm{e}^{-i H / 2}\right)\right) \mathrm{e}^{-\phi / 2} \mathrm{e}^{i k \cdot X}, \\
&\left.+\sqrt{2} \alpha^{\prime} \not k^{\dot{b} a}\left(S_{a} \partial \lambda^{i} \mathrm{e}^{i H / 2}-2 S_{a} \lambda^{i} \partial \mathrm{e}^{i H / 2}\right)\right) \mathrm{e}^{-\phi / 2} \mathrm{e}^{i k \cdot X} .
\end{aligned}
$$

Again, there is a spurious fermion which can be used to remove some internal SCFT fields from the vertex operators:

$$
\begin{aligned}
& V_{\Theta(\mathrm{sp})}^{\left(-\frac{1}{2}\right)} \sim \Theta_{i}^{a} {\left[\left(k_{a \dot{b}} \bar{\sigma}_{\mu}^{\dot{b} b}+4 k_{\mu} \delta_{a}^{b}\right) i \partial X^{\mu} S_{b} \lambda^{i} \mathrm{e}^{i H / 2}-2 \sqrt{2}\left(\alpha^{\prime} k^{\mu} \not k_{a \dot{b}}+\frac{1}{4} \sigma_{a \dot{b}}^{\mu}\right) S_{\mu}^{\dot{b}} \lambda^{i} \mathrm{e}^{i H / 2}\right.} \\
&+6 \partial S_{a} \lambda^{i} \mathrm{e}^{i H / 2}+4 S_{a} \partial \lambda^{i} \mathrm{e}^{i H / 2}+4 S_{a} \lambda^{i} \partial \mathrm{e}^{i H / 2} \\
&\left.+2 \sqrt{2 \alpha^{\prime}} \not k_{a \dot{b}} S^{\dot{b}} g^{i} \mathrm{e}^{i H / 2}-\sqrt{2} \not k_{a \dot{b}} i \partial Z^{+} S^{\dot{b}} \lambda^{i} \mathrm{e}^{-i H / 2}\right] \mathrm{e}^{-\phi / 2} \mathrm{e}^{i k \cdot X}, \\
& V_{\bar{\Theta}(\mathrm{sp})}^{\left(-\frac{1}{2}\right)} \sim \bar{\Theta}_{\dot{b}, i}\left[\left(k^{\dot{b} a} \sigma_{a \dot{a}}^{\mu}+4 k^{\mu} \delta_{\dot{a}}^{\dot{b}}\right) i \partial X_{\mu} S^{\dot{a}} \lambda^{i} \mathrm{e}^{-i H / 2}-2 \sqrt{2}\left(\alpha^{\prime} k_{\mu} \not k^{\dot{a} a}+\frac{1}{4} \bar{\sigma}_{\mu}^{\dot{b} a}\right) S_{a}^{\mu} \lambda^{i} \mathrm{e}^{-i H / 2}\right. \\
&+6 \partial S^{\dot{b}} \lambda^{i} \mathrm{e}^{-i H / 2}+4 S^{\dot{b}} \partial \lambda^{i} \mathrm{e}^{-i H / 2}+4 S^{\dot{b}} \lambda^{i} \partial \mathrm{e}^{-i H / 2} \\
&\left.+2 \sqrt{2 \alpha^{\prime}} \not k^{\dot{b} a} S_{a} g^{i} \mathrm{e}^{-i H / 2}-\sqrt{2} \not k^{\dot{b} a} i \partial Z^{-} S_{a} \lambda^{i} \mathrm{e}^{i H / 2}\right] \mathrm{e}^{-\phi / 2} \mathrm{e}^{i k \cdot X} .
\end{aligned}
$$

\subsection{SUSY transformations}

The charges of $\mathcal{N}=2$ SUSY are spinors of the internal $S U(2)$ R-symmetry and therefore carry an extra index $i$. In this sector, universal states at the first mass level split into three separate massive supermultiplets - a spin two multiplet $\left\{\alpha, \chi, \bar{\chi}, d, \xi, \beta^{ \pm}, s, \bar{s}, \phi\right\}$ as well as two spin one multiplets $\left\{\omega^{-}, \bar{a}, \bar{r}, \Phi^{+}, \zeta^{-}, \Omega_{A}^{-}\right\}$and $\left\{\omega^{+}, a, r, \Phi^{-}, \zeta^{+}, \Omega_{A}^{+}\right\}$, see figure 7 below for their structure. 


$$
\begin{aligned}
& \zeta^{+} \longleftrightarrow r_{i}^{b} \longleftrightarrow \omega_{\mu}^{+} \oplus \Omega_{A}^{+} \longleftrightarrow a_{i}^{b} \longleftrightarrow \Phi^{-} \\
& \beta_{\mu}^{+} \longleftrightarrow \chi_{\mu, i}^{a} \oplus \bar{s}_{\dot{b}, i} \longleftrightarrow \begin{array}{c}
\alpha_{\mu \nu} \oplus d_{\mu}^{A} \\
\oplus \xi_{\mu} \oplus \phi
\end{array} \longleftrightarrow \bar{\chi}_{\dot{b}, i}^{\mu} \oplus s_{i}^{a} \longleftrightarrow \beta_{\mu}^{-} \\
& \Phi^{+} \longleftrightarrow \bar{a}_{\dot{b}, i} \longleftrightarrow \omega_{\mu}^{-} \oplus \Omega_{A}^{-} \longleftrightarrow \bar{r}_{\dot{b}, i} \longleftrightarrow \zeta^{-}
\end{aligned}
$$

Figure 7: Three disconnected $\mathcal{N}=2$ SUSY multiplets

\subsubsection{SUSY variation of the spin two supermultiplet}

The spin two multiplet includes a spin two boson $\alpha_{\mu \nu}$, six vectors $\xi_{\mu}, d_{\mu}^{A=1,2,3}, \beta_{\mu}^{ \pm}$, one scalar $\phi$, two spin- $\frac{3}{2}$ fermions $\chi_{\mu}^{a}, \bar{\chi}_{\mu, \dot{a}}$ and two spin- $\frac{1}{2}$ fermions $s^{a}, \bar{s}_{\dot{a}}$. Their SUSY transformations are:

$$
\begin{gathered}
{\left[\eta_{i}^{a} \mathcal{Q}_{a}^{\left(+\frac{1}{2}\right), i}, V_{\alpha}^{(-1)}\right]=V_{\chi}^{\left(-\frac{1}{2}\right)}\left(\chi_{\mu, i}^{b}=\frac{1}{\sqrt{2}} \eta_{i}^{a} \alpha_{\mu \nu}\left(\not k \bar{\sigma}^{\nu}\right)_{a}^{b}\right),} \\
{\left[\bar{\eta}_{\dot{a}, i} \overline{\mathcal{Q}}^{\left(+\frac{1}{2}\right), \dot{a}, i}, V_{\alpha}^{(-1)}\right]=V_{\bar{\chi}}^{\left(-\frac{1}{2}\right)}\left(\bar{\chi}_{\mu, \dot{a}, i}=\frac{1}{\sqrt{2}} \bar{\eta}_{\dot{a}, i} \alpha_{\mu \nu}\left(\not k \sigma^{\nu}\right)^{\dot{a}}{ }_{\dot{b}}\right) .}
\end{gathered}
$$

For the four spin one fields, we have the following results - the SUSY variations of $\xi_{\mu}$ field read,

$$
\begin{aligned}
{\left[\eta_{i}^{a} \mathcal{Q}_{a}^{\left(+\frac{1}{2}\right), i}, V_{\xi}^{(-1)}\right] } & =V_{\chi}^{\left(-\frac{1}{2}\right)}\left(\chi_{\mu, i}^{b}=-\frac{1}{6 \sqrt{\alpha^{\prime}}} \eta_{i}^{a}\left[3 \xi_{\mu} \delta_{a}^{b}+\left(\Varangle \bar{\sigma}_{\mu}+\alpha^{\prime} \Varangle \not k k_{\mu}\right)_{a}^{b}\right]\right) \\
& +V_{\bar{s}}^{\left(-\frac{1}{2}\right)}\left(\bar{s}_{\dot{a}, i}=-\frac{1}{\sqrt{3 \alpha^{\prime}}} \eta_{i}^{a} \oiint_{a \dot{a}}\right), \\
{\left[\bar{\eta}_{\dot{a}, i} \overline{\mathcal{Q}}^{\left(+\frac{1}{2}\right), \dot{a}, i}, V_{\xi}^{(-1)}\right] } & =V_{\bar{\chi}}^{\left(-\frac{1}{2}\right)}\left(\bar{\chi}_{\mu, \dot{b}, i}=\frac{1}{6 \sqrt{\alpha^{\prime}}} \bar{\eta}_{\dot{a}, i}\left[3 \xi_{\mu} \delta_{\dot{b}}^{\dot{a}}+\left(\Varangle \bar{\sigma}_{\mu}+\alpha^{\prime} \Varangle \not k k_{\mu}\right)_{\dot{b}}^{\dot{a}}\right]\right) \\
& +V_{s}^{\left(-\frac{1}{2}\right)}\left(s_{i}^{a}=\frac{1}{\sqrt{3 \alpha^{\prime}}} \bar{\eta}_{\dot{a}, i} \dot{\phi}^{\dot{a} a}\right),
\end{aligned}
$$

the $S U(2)$ triplet $d_{\mu}^{A}$ transforms to,

$$
\begin{aligned}
{\left[\eta_{i}^{a} \mathcal{Q}_{a}^{\left(+\frac{1}{2}\right), i}, V_{d}^{(-1)}\right] } & =V_{\chi}^{\left(-\frac{1}{2}\right)}\left(\chi_{\mu, j}^{b}=-\frac{1}{3 \sqrt{2 \alpha^{\prime}}} \eta_{i}^{a}\left[3 d_{\mu}^{A} \delta_{a}^{b}+\left(\not d^{A} \bar{\sigma}_{\mu}+\alpha^{\prime} k_{\mu} \not d^{A} \not k\right)_{a}^{b}\right]\left(\tau_{A}\right)_{j}^{i}\right) \\
& +V_{\bar{s}}^{\left(-\frac{1}{2}\right)}\left(\bar{s}_{\dot{a}, j}=\frac{1}{\sqrt{6 \alpha^{\prime}}} \eta_{i}^{a} \phi_{a \dot{a}}^{A}\left(\tau_{A}\right)^{i}{ }_{j}\right), \\
{\left[\bar{\eta}_{\dot{a}, i} \overline{\mathcal{Q}}^{\left(+\frac{1}{2}\right), \dot{a}, i}, V_{d}^{(-1)}\right] } & =V_{\bar{\chi}}^{\left(-\frac{1}{2}\right)}\left(\bar{\chi}_{\mu, \dot{b}, i}=-\frac{1}{3 \sqrt{2 \alpha^{\prime}}} \bar{\eta}_{\dot{a}, i}\left[3 d_{\mu}^{A} \delta^{\dot{a}}{ }_{\dot{b}}+\left(\not d^{A} \bar{\sigma}_{\mu}+\alpha^{\prime} k_{\mu} \not d^{A} \not k\right)^{\dot{a}}{ }_{\dot{b}}\right]\left(\tau_{A}\right)^{i}{ }_{j}\right) \\
& +V_{s}^{\left(-\frac{1}{2}\right)}\left(s_{j}^{a}=\frac{1}{\sqrt{6 \alpha^{\prime}}} \bar{\eta}_{\dot{a}, i} \phi^{A, \dot{a} a}\left(\tau_{A}\right)^{i}{ }_{j}\right),
\end{aligned}
$$

and the complex vectors $\beta_{\mu}^{ \pm}$are varied to ${ }^{18}$

$$
\left[\eta_{i}^{a} \mathcal{Q}_{a}^{\left(+\frac{1}{2}\right), i}, V_{\beta^{+}}^{(-1)}\right]=0,
$$

\footnotetext{
${ }^{18}$ Cocycles would introduce additional minus signs in the computations (and several analogous ones at later points). However, we are able to eliminate these extra minus signs in a consistent way.
} 


$$
\begin{aligned}
{\left[\bar{\eta}_{b, i} \overline{\mathcal{Q}}^{\left(+\frac{1}{2}\right), \dot{b}, i}, V_{\beta^{+}}^{(-1)}\right] } & =V_{\chi}^{\left(-\frac{1}{2}\right)}\left(\chi_{\mu, i}^{b}=\frac{1}{3} \bar{\eta}_{\dot{b}, i}\left[3 \beta_{\mu}^{+} \not k^{\dot{b} b}-\left(k_{\mu} \beta^{+}+\not \beta^{+} \not k \bar{\sigma}_{\mu}\right)^{\dot{b} b}\right]\right) \\
& +V_{\bar{s}}^{\left(-\frac{1}{2}\right)}\left(\bar{s}_{\dot{s}, i}=\frac{1}{\sqrt{3}} \bar{\eta}_{\dot{b}, i}\left(\beta^{+} \not k\right)^{\dot{b}}\right), \\
{\left[\eta_{i}^{b} \mathcal{Q}_{b}^{\left(+\frac{1}{2}\right), i}, V_{\beta^{-}}^{(-1)}\right] } & =V_{\bar{\chi}}^{\left(-\frac{1}{2}\right)}\left(\bar{\chi}_{\mu, \dot{b}, i}=\frac{1}{3} \eta_{i}^{b}\left[3 \beta_{\mu}^{-} \not k_{b \dot{b}}-\left(k_{\mu} \beta^{-}+\not \beta^{-} \not k \sigma_{\mu}\right)_{b \dot{b}}\right]\right) \\
& +V_{s}^{\left(-\frac{1}{2}\right)}\left(s_{i}^{c}=\frac{1}{\sqrt{3}} \eta_{i}^{b}\left(\beta^{-} \not k^{c}\right)_{b}^{c}\right), \\
{\left[\bar{\eta}_{\dot{a}, i} \overline{\mathcal{Q}}^{\left(+\frac{1}{2}\right), \dot{a}, i}, V_{\beta^{-}}^{(-1)}\right] } & =0 .
\end{aligned}
$$

The SUSY action on the unique scalar field $\phi$ is given by

$$
\begin{aligned}
{\left[\eta_{i}^{a} \mathcal{Q}_{a}^{\left(+\frac{1}{2}\right), i}, V_{\phi}^{(-1)}\right] } & =V_{\bar{s}}^{\left(-\frac{1}{2}\right)}\left(\bar{s}_{\dot{a}, i}=\frac{1}{\sqrt{2}} \phi \eta_{i}^{a} \not k_{a \dot{a}}\right), \\
{\left[\bar{\eta}_{\dot{a}, i} \overline{\mathcal{Q}}^{\left(+\frac{1}{2}\right), \dot{a}, i}, V_{\phi}^{(-1)}\right] } & =V_{s}^{\left(-\frac{1}{2}\right)}\left(s_{i}^{a}=\frac{1}{\sqrt{2}} \phi \bar{\eta}_{\dot{a}, i} k^{\dot{a} a}\right) .
\end{aligned}
$$

Now we turn to analyze the fermionic states. For $\chi$ and $\bar{\chi}$ at spin- $\frac{3}{2}$, we have SUSY relations,

$$
\begin{aligned}
{\left[\eta_{i}^{a} \mathcal{Q}_{a}^{\left(-\frac{1}{2}\right), i}, V_{\chi}^{\left(-\frac{1}{2}\right)}\right] } & =V_{\beta^{+}}^{(-1)}\left(\beta_{\mu}^{+}=\eta_{i}^{a} \chi_{\mu, a, i}\right) \\
{\left[\bar{\eta}_{\dot{a}, i} \overline{\mathcal{Q}}^{\left(-\frac{1}{2}\right), \dot{a}, i}, V_{\chi}^{\left(-\frac{1}{2}\right)}\right] } & =V_{\alpha}^{(-1)}\left(\alpha_{\mu \nu}=\frac{1}{\sqrt{2}} \bar{\eta}_{\dot{a}, i}\left(\bar{\sigma}_{(\mu}^{\dot{a} a} \chi_{\nu), a}^{i}+\alpha^{\prime} k^{\dot{a} a} \chi_{(\mu|, a|}^{i} k_{\nu)}\right)\right) \\
& +V_{\xi}^{(-1)}\left(\xi_{\mu}=-\frac{\sqrt{\alpha^{\prime}}}{2} \bar{\eta}_{\dot{a}, i} k^{\dot{k} a} \chi_{\mu, a}^{i}\right) \\
& +V_{d}^{(-1)}\left(d_{\mu}^{A}=\sqrt{\frac{\alpha^{\prime}}{2}} \bar{\eta}_{\dot{a}, i} k^{\dot{a} a} \chi_{\mu, a, j}\left(\tau^{A} \varepsilon\right)^{i j}\right)
\end{aligned}
$$

and

$$
\begin{aligned}
{\left[\eta_{i}^{a} \mathcal{Q}_{a}^{\left(-\frac{1}{2}\right), i}, V_{\bar{\chi}}^{\left(-\frac{1}{2}\right)}\right] } & =V_{\alpha}^{(-1)}\left(\alpha_{\mu \nu}=\frac{1}{\sqrt{2}} \eta_{i}^{a}\left(\sigma_{(\mu|a \dot{a}|} \bar{\chi}_{\nu)}^{\dot{a}, i}+\alpha^{\prime} k_{a \dot{a}} \bar{\chi}_{(\mu}^{\dot{a}, i} k_{\nu)}\right)\right) \\
& +V_{\xi}^{(-1)}\left(\xi_{\mu}=\frac{\sqrt{\alpha^{\prime}}}{2} \eta_{i}^{a} k_{a \dot{a}} \bar{\chi}_{\mu}^{\dot{a}, i}\right) \\
& +V_{d}^{(-1)}\left(d_{\mu}^{A}=\sqrt{\frac{\alpha^{\prime}}{2}} \eta_{i}^{a} k_{a \dot{a}} \bar{\chi}_{\mu, j}^{\dot{a}}\left(\tau^{A} \varepsilon\right)^{i j}\right), \\
{\left[\bar{\eta}_{\dot{a}, i} \overline{\mathcal{Q}}^{\left(-\frac{1}{2}\right), \dot{a}, i}, V_{\bar{\chi}}^{\left(-\frac{1}{2}\right)}\right] } & =V_{\beta^{-}}^{(-1)}\left(\beta_{\mu}^{-}=\bar{\eta}_{\dot{a}, i} \bar{\chi}_{\mu}^{\dot{a}, i}\right) .
\end{aligned}
$$

The spin- $\frac{1}{2}$ states $s$ and $\bar{s}$, on the other hand, transform to

$$
\begin{aligned}
{\left[\eta_{i}^{a} \mathcal{Q}_{a}^{\left(-\frac{1}{2}\right), i}, V_{s}^{\left(-\frac{1}{2}\right)}\right] } & =V_{\xi}^{(-1)}\left(\xi_{\mu}=\sqrt{\frac{\alpha^{\prime}}{3}} \eta_{i}^{a}\left[k_{\mu} \delta_{a}^{b}+\left(\sigma_{\mu} \not k\right)_{a}{ }^{b}\right] s_{b}^{i}\right) \\
& +V_{d}^{(-1)}\left(d_{\mu}^{A}=\sqrt{\frac{\alpha^{\prime}}{6}} \eta_{i}^{a}\left[k_{\mu} \delta_{a}^{b}+\left(\sigma_{\mu} \not\right)_{a}{ }_{a}\right] s_{b, j}\left(\tau^{A} \varepsilon\right)^{i j}\right)
\end{aligned}
$$




$$
\begin{gathered}
+V_{\phi}^{(-1)}\left(\phi=\frac{1}{\sqrt{2}} \eta_{i}^{a} s_{a}^{i}\right) \\
{\left[\bar{\eta}_{\dot{a}, i} \overline{\mathcal{Q}}^{\left(-\frac{1}{2}\right), \dot{a}, i}, V_{s}^{\left(-\frac{1}{2}\right)}\right]=V_{\beta^{-}}^{(-1)}\left(\beta_{\mu}^{-}=-\frac{1}{\sqrt{3}} \bar{\eta}_{\dot{a}, i}\left(\bar{\sigma}_{\mu}^{\dot{a} a}+\alpha^{\prime} k_{\mu} k^{\dot{a} a}\right) s_{a}^{i}\right),}
\end{gathered}
$$

and

$$
\begin{aligned}
{\left[\eta_{i}^{a} \mathcal{Q}_{a}^{\left(-\frac{1}{2}\right), i}, V_{\bar{s}}^{\left(-\frac{1}{2}\right)}\right] } & =V_{\beta^{+}}^{(-1)}\left(\beta_{\mu}^{+}=-\frac{1}{\sqrt{3}} \eta_{i}^{a}\left(\sigma_{\mu a \dot{a}}+\alpha^{\prime} k_{\mu} \not k_{a \dot{a}}\right) \bar{s}^{\dot{a}, i}\right), \\
{\left[\bar{\eta}_{\dot{a}, i} \overline{\mathcal{Q}}^{\left(-\frac{1}{2}\right), \dot{a}, i}, V_{\bar{s}}^{\left(-\frac{1}{2}\right)}\right] } & =V_{\xi}^{(-1)}\left(\xi_{\mu}=\sqrt{\frac{\alpha^{\prime}}{3}} \bar{\eta}_{\dot{a}, i}\left[k_{\mu} \delta^{\dot{a}}{ }_{\dot{b}}+\left(\bar{\sigma}_{\mu} \not k\right)^{\dot{a}}\right] \bar{s}^{\dot{b}, i}\right) \\
& +V_{d}^{(-1)}\left(d_{\mu}^{A}=\sqrt{\frac{\alpha^{\prime}}{6}} \bar{\eta}_{\dot{a}, i}\left[k_{\mu} \delta^{\dot{a}}{ }_{\dot{b}}+\left(\bar{\sigma}_{\mu} \not k\right)^{\dot{a}}{ }_{\dot{b}}\right] \bar{s}^{\dot{b}, i}\left(\tau^{A} \varepsilon\right)^{i j}\right) \\
& +V_{\phi}^{(-1)}\left(\phi=\frac{1}{\sqrt{2}} \bar{\eta}_{\dot{a}, i} \bar{r}_{+}^{\dot{a}, i}\right) .
\end{aligned}
$$

\subsubsection{SUSY variation of the spin one supermultiplets}

The first spin one multiplet $\left\{\omega^{-}, \bar{a}, \bar{r}, \Phi^{+}, \zeta^{-}, \Omega_{A}^{-}\right\}$contains one vector $\omega_{\mu}^{-}$, two right-handed fermions $\bar{a}_{\dot{b}}$ and $\bar{r}_{\dot{b}}$ of spin $1 / 2$ each, and three scalars $\Phi^{+}, \zeta^{-}$and $\Omega_{A}^{-}$. The SUSY relations for the spin one $\omega_{\mu}^{-}$read,

$$
\begin{gathered}
{\left[\eta_{i}^{b} \mathcal{Q}_{b}^{\left(+\frac{1}{2}\right), i}, V_{\omega^{-}}^{(-1)}\right]=V_{\bar{a}}^{(-1)}\left(\bar{a}_{\dot{b}, i}=-\frac{1}{\sqrt{2 \alpha^{\prime}}} \eta_{i}^{b} \psi_{b \dot{b}}^{-}\right),} \\
{\left[\bar{\eta}_{\dot{a}, i} \overline{\mathcal{Q}}^{\left(+\frac{1}{2}\right), \dot{a}, i}, V_{\omega^{-}}^{(-1)}\right]=V_{\bar{r}}^{\left(+\frac{1}{2}\right)}\left(\bar{r}_{\dot{b}, i}=-\frac{1}{\sqrt{2}} \bar{\eta}_{\dot{a}, i}\left(\psi^{-} \not k\right)^{\dot{a}}{ }_{\dot{b}}\right) .}
\end{gathered}
$$

For the fermions $\bar{a}_{\dot{b}}$ and $\bar{r}_{\dot{b}}$, we have,

$$
\begin{aligned}
{\left[\eta_{i}^{b} \mathcal{Q}_{b}^{\left(-\frac{1}{2}\right), i}, V_{\bar{a}}^{\left(-\frac{1}{2}\right)}\right] } & =V_{\Phi^{+}}^{(-1)}\left(\Phi^{+}=\sqrt{\alpha^{\prime}} \eta_{i}^{b} \hbar_{b \dot{b}} \bar{a}^{\dot{b}, i}\right) \\
{\left[\bar{\eta}_{\dot{b}, i} \overline{\mathcal{Q}}^{\left(-\frac{1}{2}\right), \dot{b}, i}, V_{\bar{a}}^{\left(-\frac{1}{2}\right)}\right] } & =V_{\omega^{-}}^{(-1)}\left(\omega_{\mu}^{-}=\sqrt{\frac{\alpha^{\prime}}{2}} \bar{\eta}_{\dot{b}, i}\left[k_{\mu} \delta_{\dot{c}}^{\dot{b}}+\left(\not k \sigma_{\mu}\right)_{\dot{c}}^{\dot{b}}\right] \bar{a}^{\dot{c}, i}\right) \\
& +V_{\Omega^{-}}^{(-1)}\left(\Omega_{A}^{-}=-\frac{1}{\sqrt{2}} \bar{\eta}_{\dot{b}, i}\left(\tau_{A} \varepsilon\right)^{i j} \bar{a}_{j}^{\dot{b}}\right),
\end{aligned}
$$

and

$$
\begin{aligned}
{\left[\eta_{i}^{a} \mathcal{Q}_{a}^{\left(-\frac{1}{2}\right), i}, V_{\bar{r}}^{\left(-\frac{1}{2}\right)}\right] } & =V_{\omega^{-}}^{(-1)}\left(\omega_{\mu}^{-}=\frac{1}{\sqrt{2}} \eta_{i}^{a}\left(\sigma_{\mu a \dot{a}}+\alpha^{\prime} k_{\mu} k_{a \dot{a}}\right) \bar{r}^{\dot{a}, i}\right) \\
& +V_{\Omega^{-}}^{(-1)}\left(\Omega_{A}^{-}=\sqrt{\frac{\alpha^{\prime}}{2}} \eta_{i}^{a} \not k_{a \dot{a}} \bar{r}_{j}^{\dot{a}}\left(\tau_{A} \varepsilon\right)^{i j}\right), \\
{\left[\bar{\eta}_{\dot{a}, i} \overline{\mathcal{Q}}^{\left(-\frac{1}{2}\right), \dot{a}, i}, V_{\bar{r}}^{\left(-\frac{1}{2}\right)}\right] } & =V_{\zeta^{-}}^{(-1)}\left(\zeta^{-}=\bar{\eta}_{\dot{a}, i} \bar{r}^{\dot{a}, i}\right) .
\end{aligned}
$$


The results for the scalar fields are:

$$
\begin{aligned}
{\left[\eta_{i}^{b} \mathcal{Q}_{b}^{\left(+\frac{1}{2}\right), i}, V_{\Phi^{+}}^{(-1)}\right] } & =0 \\
{\left[\bar{\eta}_{\dot{b}, i} \overline{\mathcal{Q}}^{\left(+\frac{1}{2}\right), \dot{b}, i}, V_{\Phi^{+}}^{(-1)}\right] } & =V_{\bar{a}}^{\left(-\frac{1}{2}\right)}\left(\bar{a}_{\dot{b}, i}=-\alpha^{\prime-\frac{1}{2}} \Phi^{+} \bar{\eta}_{\dot{b}, i}\right)
\end{aligned}
$$

and

$$
\begin{aligned}
{\left[\eta_{i}^{a} \mathcal{Q}_{a}^{\left(+\frac{1}{2}\right), i}, V_{\zeta^{-}}^{(-1)}\right] } & =V_{\bar{r}}^{\left(-\frac{1}{2}\right)}\left(\bar{r}_{\dot{a}, i}=\zeta^{-} \eta_{i}^{a} \not k_{a \dot{a}}\right), \\
{\left[\bar{\eta}_{\dot{a}, i} \overline{\mathcal{Q}}^{\left(+\frac{1}{2}\right), \dot{a}, i}, V_{\zeta^{-}}^{(-1)}\right] } & =0
\end{aligned}
$$

and

$$
\begin{gathered}
{\left[\eta_{i}^{b} \mathcal{Q}_{b}^{\left(+\frac{1}{2}\right), i}, V_{\Omega^{-}}^{(-1)}\right]=V_{\bar{a}}^{\left(-\frac{1}{2}\right)}\left(\bar{a}_{\dot{b}, j}=-\frac{1}{\sqrt{2}} \eta_{i}^{b} k_{b \dot{b}} \Omega_{A}^{-}\left(\tau^{A}\right)^{i}{ }_{j}\right)} \\
{\left[\bar{\eta}_{\dot{a}, i} \overline{\mathcal{Q}}^{\left(+\frac{1}{2}\right), \dot{a}, i}, V_{\Omega^{-}}^{(-1)}\right]=V_{\bar{r}}^{\left(-\frac{1}{2}\right)}\left(\bar{r}_{\dot{a}, j}=\frac{1}{\sqrt{2 \alpha^{\prime}}} \bar{\eta}_{\dot{a}, i} \Omega_{A}^{-}\left(\tau^{A}\right)^{i}{ }_{j}\right) .}
\end{gathered}
$$

The second spin one multiplet $\left\{\omega^{+}, a, r, \Phi^{-}, \zeta^{+}, \Omega_{A}^{+}\right\}$is just the complex conjugate of the former, so let us simply list the analogous SUSY transformations:

$$
\begin{aligned}
{\left[\eta_{i}^{a} \mathcal{Q}_{a}^{\left(+\frac{1}{2}\right), i}, V_{\omega^{+}}^{(-1)}\right] } & =V_{r}^{\left(+\frac{1}{2}\right)}\left(r_{i}^{b}=-\frac{1}{\sqrt{2}} \eta_{i}^{a}\left(\psi^{+} \not k\right)_{a}^{b}\right), \\
{\left[\bar{\eta}_{\dot{b}, i} \overline{\mathcal{Q}}^{\left(+\frac{1}{2}\right), \dot{b}, i}, V_{\omega^{+}}^{(-1)}\right] } & =V_{a}^{(-1)}\left(a_{i}^{b}=-\frac{1}{\sqrt{2 \alpha^{\prime}}} \bar{\eta}_{\dot{b}, i} \psi^{+, \dot{b} b}\right), \\
{\left[\eta_{i}^{b} \mathcal{Q}_{b}^{\left(-\frac{1}{2}\right), i}, V_{a}^{\left(-\frac{1}{2}\right)}\right] } & =V_{\omega^{+}}^{(-1)}\left(\omega_{\mu}^{+}=\sqrt{\frac{\alpha^{\prime}}{2}} \eta_{i}^{b}\left[k_{\mu} \delta_{b}^{c}+\left(\not k \bar{\sigma}_{\mu}\right)_{b}{ }^{c}\right] a_{c}^{i}\right) \\
& +V_{\Omega^{+}}^{(-1)}\left(\Omega_{A}^{+}=-\frac{1}{\sqrt{2}} \eta_{i}^{b}\left(\tau_{A} \varepsilon\right)^{i j} a_{b, j}\right), \\
{\left[\bar{\eta}_{\dot{b}, i} \overline{\mathcal{Q}}^{\left(-\frac{1}{2}\right), \dot{b}, i}, V_{a}^{\left(-\frac{1}{2}\right)}\right] } & =V_{\Phi^{-}}^{(-1)}\left(\Phi^{-}=\sqrt{\alpha^{\prime}} \bar{\eta}_{\dot{b}, i} \not k^{\dot{b} b} a_{b}^{i}\right) \\
{\left[\eta_{i}^{a} \mathcal{Q}_{a}^{\left(-\frac{1}{2}\right), i}, V_{r}^{\left(-\frac{1}{2}\right)}\right] } & =V_{\zeta^{+}}^{(-1)}\left(\zeta^{+}=\eta_{i}^{a} r_{a}^{i}\right) \\
{\left[\bar{\eta}_{\dot{a}, i} \overline{\mathcal{Q}}^{\left(-\frac{1}{2}\right), \dot{a}, i}, V_{r}^{\left(-\frac{1}{2}\right)}\right] } & =V_{\omega^{+}}^{(-1)}\left(\omega_{\mu}^{+}=\frac{1}{\sqrt{2}} \bar{\eta}_{\dot{a}, i}\left(\bar{\sigma}_{\mu}^{\dot{a} a}+\alpha^{\prime} k_{\mu} k^{\dot{a} a}\right) r_{a}^{i}\right) \\
& +V_{\Omega^{+}}^{(-1)}\left(\Omega_{A}^{+}=\sqrt{\frac{\alpha^{\prime}}{2}} \bar{\eta}_{\dot{a}, i} \not k^{\dot{a} a} r_{a, j}\left(\tau_{A} \varepsilon\right)^{i j}\right), \\
{\left[\eta_{i}^{b} \mathcal{Q}_{b}^{\left(+\frac{1}{2}\right), i}, V_{\Phi^{-}}^{(-1)}\right] } & =V_{a}^{\left(-\frac{1}{2}\right)}\left(a_{i}^{b}=-\alpha^{\prime-\frac{1}{2}} \Phi^{-} \eta_{i}^{b}\right) \\
{\left[\bar{\eta}_{\dot{b}, i} \overline{\mathcal{Q}}^{\left(+\frac{1}{2}\right), \dot{b}, i}, V_{\Phi^{-}}^{(-1)}\right] } & =0 \\
{\left[\eta_{i}^{a} \mathcal{Q}_{a}^{\left(+\frac{1}{2}\right), i}, V_{\zeta^{+}}^{(-1)}\right] } & =0, \\
\left.\bar{\eta}_{\dot{a}, i}^{\left(+\frac{1}{2}\right), \dot{a}, i}, V_{\zeta^{+}}^{(-1)}\right] & =V_{r}^{\left(-\frac{1}{2}\right)}\left(r_{i}^{a}=\zeta^{+} \bar{\eta}_{\dot{a}, i} k^{\dot{a} a}\right),
\end{aligned}
$$




$$
\begin{gathered}
{\left[\eta_{i}^{a} \mathcal{Q}_{a}^{\left(+\frac{1}{2}\right), i}, V_{\Omega^{+}}^{(-1)}\right]=V_{r}^{\left(-\frac{1}{2}\right)}\left(r_{j}^{a}=\frac{1}{\sqrt{2 \alpha^{\prime}}} \eta_{i}^{a} \Omega_{A}^{+}\left(\tau^{A}\right)^{i}{ }_{j}\right),} \\
{\left[\bar{\eta}_{\dot{b}, i} \overline{\mathcal{Q}}^{\left(+\frac{1}{2}\right), \dot{b}, i}, V_{\Omega^{+}}^{(-1)}\right]=V_{a}^{\left(-\frac{1}{2}\right)}\left(a_{j}^{b}=-\frac{1}{\sqrt{2}} \bar{\eta}_{\dot{b}, i} k^{\dot{b} b} \Omega_{A}^{+}\left(\tau^{A}\right)^{i}{ }_{j}\right) .}
\end{gathered}
$$

\section{$7 \quad$ Helicity structure of massive on-shell multiplets}

In this section, we apply the massive version of the spinor helicity formalism [22, 23] to obtain a refined understanding of the structure of the previously constructed SUSY multiplets. A brief summary of the spinor techniques is collected in appendix C, including the explicit form of massive wavefunctions associated with different spin components. The spin quantization axis is chosen covariantly by decomposing the timelike momentum $k$ into two arbitrary light-like reference momenta $p$ and $q$ :

$$
k^{\mu}=p^{\mu}+q^{\mu}, \quad k^{2}=-m^{2}=2 p q, \quad p^{2}=q^{2}=0
$$

As was explained in detail in [24], the supercharges can be expanded in the basis of the momentum spinors $p_{a}, p^{* \dot{a}}$ and $q_{a}, q^{* \dot{a}}$ defined by $p_{\mu} \sigma_{a \dot{a}}^{\mu}=-p_{a} p_{\dot{a}}^{*}$ and $q_{\mu} \sigma_{a \dot{a}}^{\mu}=-q_{a} q_{\dot{a}}^{*}$ :

$$
\begin{aligned}
\mathcal{Q}_{a} & =\frac{[q \mathcal{Q}]}{[q p]} p_{a}+\frac{[p \mathcal{Q}]}{[p q]} q_{a}=\mathcal{Q}_{+} p_{a}+\mathcal{Q}_{-} q_{a}, \\
\overline{\mathcal{Q}}^{\dot{a}} & =\frac{\langle p \overline{\mathcal{Q}}\rangle}{\langle p q\rangle} q^{* \dot{a}}+\frac{\langle q \overline{\mathcal{Q}}\rangle}{\langle q p\rangle} p^{* \dot{a}}=\overline{\mathcal{Q}}_{+} q^{* \dot{a}}+\overline{\mathcal{Q}}_{-} p^{* \dot{a}} .
\end{aligned}
$$

This defines the supercharge components $\mathcal{Q}_{ \pm}$and $\overline{\mathcal{Q}}_{ \pm}$to be

$$
\begin{aligned}
\mathcal{Q}_{+} \equiv \frac{[q \mathcal{Q}]}{[q p]}, & \mathcal{Q}_{-} \equiv \frac{[p \mathcal{Q}]}{[p q]} \\
\overline{\mathcal{Q}}_{+} \equiv \frac{\langle p \overline{\mathcal{Q}}\rangle}{\langle p q\rangle}, & \overline{\mathcal{Q}}_{-} \equiv \frac{\langle q \overline{\mathcal{Q}}\rangle}{\langle q p\rangle} .
\end{aligned}
$$

The $\mathcal{Q}_{+}$and $\overline{\mathcal{Q}}_{+}$raise the spin quantum $j_{z}$ number along the quantization axis by $1 / 2$, while $\mathcal{Q}_{-}$and $\overline{\mathcal{Q}}_{-}$lower it by $1 / 2$. The corresponding Lorentz generator which is diagonalized with eigenvalues $j_{z}$ reads

$$
J_{z}=\frac{1}{m^{2}} \varepsilon^{\mu \nu \lambda \rho} P_{\mu} q_{\nu} M_{\lambda \rho}
$$

where $P_{\mu}$ denotes the translation operator and $M_{\lambda \rho}$ an $S O(1,3)$ rotation.

A convenient way of organizing representations of the super Poincaré group is to pick a highest weight state which is annihilated by half the supercharges - either the left-handed $\mathcal{Q}_{a}$ or the righthanded $\overline{\mathcal{Q}}^{\dot{b}}$. States with this property are referred to as (anti-)Clifford vacua, and we shall use 
the vacuum eliminated by the left-handed $\mathcal{Q}_{a}$ by convention. The rest of the supermultiplet is then constructed by applying the nontrivially acting $\overline{\mathcal{Q}}_{+}$and $\overline{\mathcal{Q}}_{-}$, see the figures in this section. In our notation, each diamond shaped diagram represents one supermultiplet. The dashed lines connecting bosonic and fermionic states indicate $\mathcal{Q}_{ \pm}$and $\overline{\mathcal{Q}}_{ \pm}$applications, and we assign the following directions:

$$
\nearrow \equiv \overline{\mathcal{Q}}_{+}, \quad \searrow \equiv \overline{\mathcal{Q}}_{-} \quad \text { and } \quad \nwarrow \equiv \mathcal{Q}_{+}, \quad \swarrow \equiv \mathcal{Q}_{-}
$$

The Clifford vacuum state being annihilated by the left-handed $\mathcal{Q}_{ \pm}$is located on the far left of the diamond, and we can construct the full supermultiplet by repeated action of $\overline{\mathcal{Q}}_{ \pm} \sqrt{19}$ In this section, we will show how $\overline{\mathcal{Q}}_{ \pm}$transform all the states in the multiplet from the left side of the diamond all the way to the right. The SUSY algebras $\left\{\mathcal{Q}_{ \pm}, \overline{\mathcal{Q}}_{\mp}\right\}=1$ and $\left\{\mathcal{Q}_{ \pm}, \overline{\mathcal{Q}}_{ \pm}\right\}=020$ imply that $\mathcal{Q}_{ \pm}$ undoes $\overline{\mathcal{Q}}_{ \pm}$applications and transforms states from right to left in the diamond.

This section starts with the $\mathcal{N}=1$ situation to illustrate the methods, and the additional features of extended SUSY are explained in the later subsections on $\mathcal{N}=2,4$ supermultiplets. To make everything simple and clear, instead of using our old notation of vertex operators in the previous sections, we will use the "ket" notation to express the states inside the diamonds. For example, the spin two boson with $j_{z}=+2$ is expressed by

$$
|\alpha,+2\rangle \equiv V_{\alpha}^{(-1)}\left(\alpha^{\mu \nu}=\frac{1}{2 m^{2}} \bar{\sigma}^{\mu \dot{a} a} \bar{\sigma}^{\nu \dot{b} b} p_{\dot{a}}^{*} q_{a} p_{\dot{b}}^{*} q_{b}\right)
$$

and a combined state $\{\alpha, d\}$ with $j_{z}=+1$ is expressed by $|\alpha \oplus d,+1\rangle$. The commutators of $\mathcal{Q}_{a}$ and $\overline{\mathcal{Q}}^{\dot{b}}$ with vertex operators are replaced by SUSY transformations acting directly on the states.

\footnotetext{
${ }^{19}$ Alternatively, we can also construct this supermultiplet starting from the anti-Clifford vacuum state on the right side of this diamond, which is eliminated by the anti-supercharge $\overline{\mathcal{Q}}_{ \pm}$, and the remaining states follow by acting $\mathcal{Q}_{ \pm}$on it.

${ }^{20}$ To show this, we simply plug the supercharge decompositions 7.2 and 7.3 into the $\mathcal{N}=1$ SUSY algebra (3.44), and obtain,

$$
\begin{aligned}
\left\{\mathcal{Q}_{a}^{\left(+\frac{1}{2}\right)}, \overline{\mathcal{Q}}^{\left(-\frac{1}{2}\right), \dot{a}}\right\} & =p_{a} p^{* \dot{a}}\left\{\mathcal{Q}_{+}, \overline{\mathcal{Q}}_{-}\right\}+p_{a} q^{* \dot{a}}\left\{\mathcal{Q}_{+}, \overline{\mathcal{Q}}_{+}\right\}+q_{a} q^{* \dot{a}}\left\{\mathcal{Q}_{-}, \overline{\mathcal{Q}}_{+}\right\}+q_{a} p^{* \dot{a}}\left\{\mathcal{Q}_{-}, \overline{\mathcal{Q}}_{-}\right\} \\
& =\left(\sigma^{\mu} \varepsilon\right)_{a}{ }^{\dot{a}} P_{\mu} \sim\left(\sigma^{\mu} \varepsilon\right)_{a}{ }^{\dot{a}} k_{\mu}=p_{a} p^{* \dot{a}}+q_{a} q^{* \dot{a}} .
\end{aligned}
$$
}

Thus we arrive at,

$$
\left(\begin{array}{ll}
\left\{\mathcal{Q}_{+}, \overline{\mathcal{Q}}_{-}\right\} & \left\{\mathcal{Q}_{+}, \overline{\mathcal{Q}}_{+}\right\} \\
\left\{\mathcal{Q}_{-}, \overline{\mathcal{Q}}_{-}\right\} & \left\{\mathcal{Q}_{-}, \overline{\mathcal{Q}}_{+}\right\}
\end{array}\right)=\left(\begin{array}{cc}
1 & 0 \\
0 & 1
\end{array}\right)
$$




\section{$7.1 \mathcal{N}=1$ supermultiplets}

According to the strategy outlined above, it suffices to evaluate the anti-supercharge components $\overline{\mathcal{Q}}_{ \pm}$on the helicity states in the $\mathcal{N}=1$ supermultiplets. The decomposition $\overline{\mathcal{Q}}^{\dot{a}}=\overline{\mathcal{Q}}_{+} q^{\dot{a} *}+\overline{\mathcal{Q}}_{-} p^{\dot{a} *}$ corresponds to the mass dimension $\left[M^{-\frac{1}{2}}\right]$ choices for $\bar{\eta}$ :

$$
\overline{\mathcal{Q}}_{ \pm}=\bar{\eta}_{\bar{a}}^{ \pm} \mathcal{Q}^{\bar{a}} \longleftrightarrow \quad \bar{\eta}_{\dot{a}}^{+}=\frac{p_{\dot{a}}^{*}}{\langle p q\rangle}, \quad \bar{\eta}_{\dot{a}}^{-}=\frac{q_{\dot{a}}^{*}}{\langle q p\rangle} .
$$

\subsubsection{Spin one half supermultiplets}

We firstly consider the $\left\{\Phi^{+}, \bar{a}, \Omega^{-}\right\}$multiplet of highest spin $1 / 2$ whose scalar Clifford vacuum $\left|\Phi^{+}\right\rangle$is eliminated by the supercharge $\mathcal{Q}^{a}$. By repeated actions of the anti-supercharge $\overline{\mathcal{Q}}_{ \pm}$on $\Phi^{+}$, we can construct the remainder of the multiplet, see figure 8 .

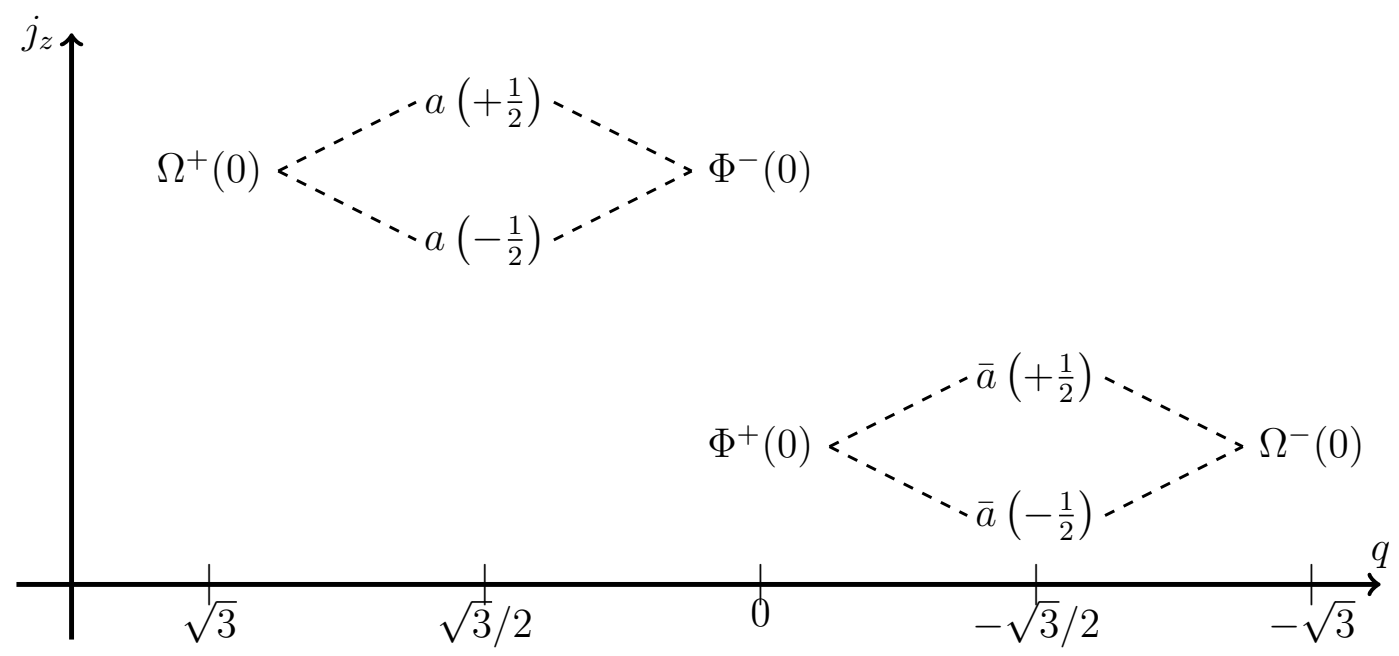

Figure 8: $\mathcal{N}=1$ SUSY multiplets with scalar Clifford vacuum: In $\mathcal{N}=1$ scenarios, the $U(1)$ charge $q$ with respect to the internal current $\mathcal{J}$ is plotted along the horizontal axis. The SUSY charges have eigenvalue $\pm \sqrt{3} / 2$ under $\mathcal{J}$ and therefore change $q$ by a fixed offset.

The spin- $\frac{1}{2}$ multiplet is the minimal massive representation of the $\mathcal{N}=1$ SUSY algebra, since it only contains four states. Very straightly, we obtain, up to a phase,

$$
\overline{\mathcal{Q}}_{ \pm}\left|\Phi^{+}, 0\right\rangle=\left|\bar{a}, \pm \frac{1}{2}\right\rangle
$$

and

$$
\overline{\mathcal{Q}}_{ \pm}\left|\bar{a}, \mp \frac{1}{2}\right\rangle=\left|\Omega^{-}, 0\right\rangle, \quad \overline{\mathcal{Q}}_{ \pm}\left|\bar{a}, \pm \frac{1}{2}\right\rangle=0
$$


The anti-Clifford vacuum $\left|\Omega^{-}\right\rangle$is then annihilated by $\overline{\mathcal{Q}}_{ \pm}$action,

$$
\overline{\mathcal{Q}}_{ \pm}\left|\Omega^{-}, 0\right\rangle=0
$$

Secondly, we consider the mirror multiplet $\left\{\Omega^{+}, a, \Phi^{-}\right\}$which is also summarized in figure 8 . Starting from the Clifford vacuum $\left|\Omega^{+}\right\rangle$, c.f. (5.37), we obtain,

$$
\overline{\mathcal{Q}}_{ \pm}\left|\Omega^{+}, 0\right\rangle=\left|a, \pm \frac{1}{2}\right\rangle
$$

and

$$
\overline{\mathcal{Q}}_{ \pm}\left|a, \mp \frac{1}{2}\right\rangle=\left|\Phi^{-}, 0\right\rangle, \quad \overline{\mathcal{Q}}_{ \pm}\left|a, \pm \frac{1}{2}\right\rangle=0
$$

\subsubsection{Spin two supermultiplet}

In addition to the two minimal spin $1 / 2$ multiplets, there is a larger multiplet $\{\alpha, \chi, \bar{\chi}, d\}$ with spins up to $j_{z}=2$ in each $\mathcal{N}=1$ scenario. All the left-handed spin $3 / 2$ states $\left|\chi, j_{z}\right\rangle$ with $-3 / 2 \leq j_{z} \leq+3 / 2$ are annihilated by $\mathcal{Q}_{a}$, c.f. (5.25). Hence, the Clifford vacuum transforms in a nontrivial $S O(1,3)$ representation. Starting from the four states $\left|\chi, j_{z}\right\rangle$, we build the full spin two multiplet by $\overline{\mathcal{Q}}_{ \pm}$application, see figure 9 . The spin- $\frac{3}{2}$ states with wavefunction $\bar{\chi}_{\dot{a}}^{\mu}$ of opposite chirality are obtained by $\left|\bar{\chi}, j_{z}\right\rangle=\overline{\mathcal{Q}}_{+} \overline{\mathcal{Q}}_{-}\left|\chi, j_{z}\right\rangle$, so they form the anti-Clifford vacua.

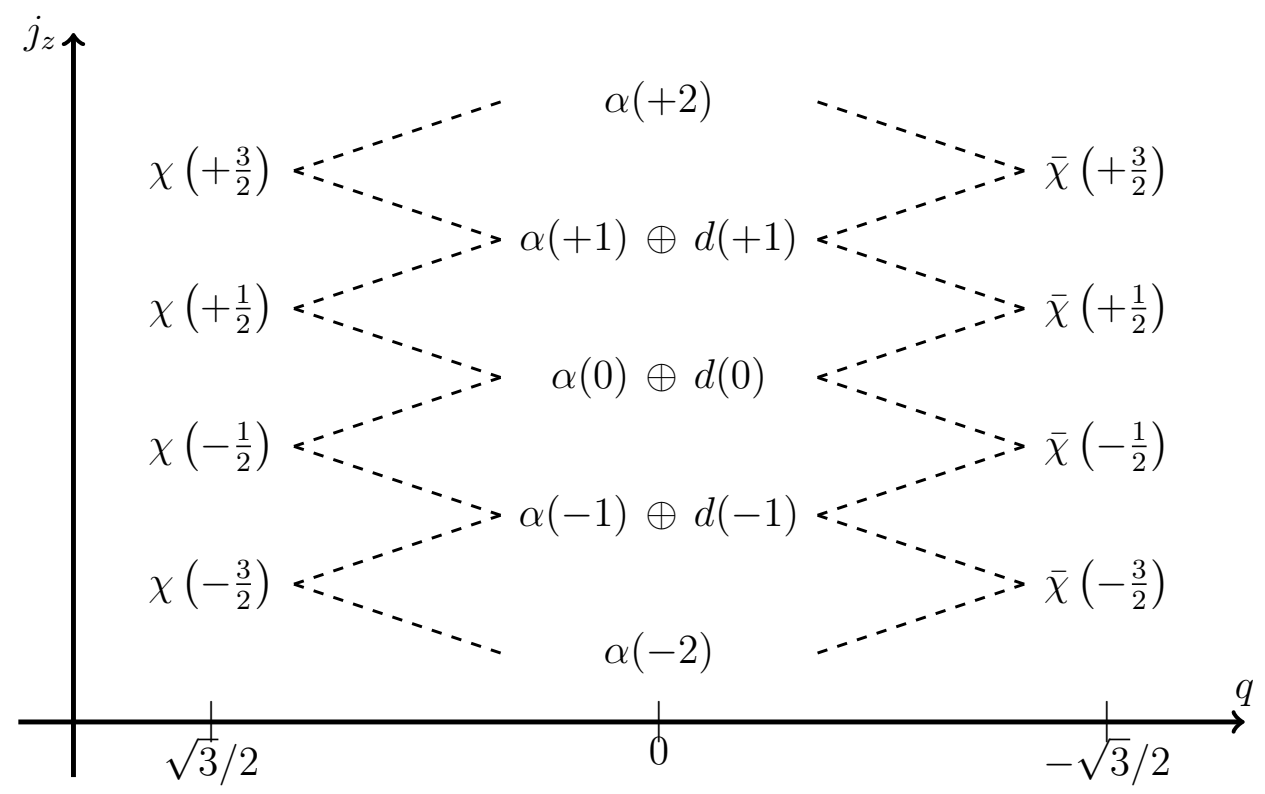

Figure 9: $\mathcal{N}=1$ SUSY multiplets with spin 3/2 Clifford vacuum

The helicity SUSY transformations are such that normalized states are either mapped to equally normalized states or annihilated. This becomes particularly interesting at the intersection points 
$\overline{\mathcal{Q}}_{-}\left|\chi, j_{z}\right\rangle \leftrightarrow \overline{\mathcal{Q}}_{+}\left|\chi, j_{z}-1\right\rangle$ within the diamond where combination states of type $|\alpha \oplus d\rangle$ arise. From the $j_{z}= \pm \frac{3}{2}$ components, we obtain

$$
\begin{aligned}
& \overline{\mathcal{Q}}_{ \pm}\left|\chi, \pm \frac{3}{2}\right\rangle=|\alpha, \pm 2\rangle, \\
& \overline{\mathcal{Q}}_{\mp}\left|\chi, \pm \frac{3}{2}\right\rangle=\frac{1}{2}|\alpha, \pm 1\rangle \pm \frac{\sqrt{3}}{2}|d, \pm 1\rangle \equiv|\alpha \pm d, \pm 1\rangle,
\end{aligned}
$$

whereas $\overline{\mathcal{Q}}_{ \pm}$action on $j_{z}= \pm \frac{1}{2}$ components yields

$$
\begin{aligned}
& \overline{\mathcal{Q}}_{ \pm}\left|\chi, \pm \frac{1}{2}\right\rangle=\frac{\sqrt{3}}{2}|\alpha, \pm 1\rangle \mp \frac{1}{2}|d, \pm 1\rangle \equiv|\alpha \mp d, \pm 1\rangle, \\
& \overline{\mathcal{Q}}_{\mp}\left|\chi, \pm \frac{1}{2}\right\rangle=\frac{1}{\sqrt{2}}|\alpha, 0\rangle \pm \frac{1}{\sqrt{2}}|d, 0\rangle \equiv|\alpha \pm d, 0\rangle .
\end{aligned}
$$

We use canonical normalization conventions for vertex operators as well as helicity wavefunctions: Let $\left|\psi, j_{z}\right\rangle$ denote some physical state with polarization tensor $\psi$ and spin component $j_{z}$ along the quantization axis. Then, $\left|\psi,+j_{z}\right\rangle$ has unit scalar product with $\left|\psi,-j_{z}\right\rangle$ and is orthogonal to all states whose wavefunction belongs to a different $S O(3)$ representation. We can see from above results that all the states on the right-hand sides of (7.15) to (7.18) have unit norm. Furthermore, we find that the combined states $|\alpha \pm d, \pm 1\rangle$ obtained from $\overline{\mathcal{Q}}_{\mp}\left|\chi, \pm \frac{3}{2}\right\rangle$ are orthogonal to $|\alpha \mp d, \pm 1\rangle$ from distinct Clifford vacuum components $\overline{\mathcal{Q}}_{ \pm}\left|\chi, \pm \frac{1}{2}\right\rangle$, as expected.

To complete the other half of the diamond, we have,

$$
\overline{\mathcal{Q}}_{ \pm}|\alpha,+2\rangle=0, \quad \overline{\mathcal{Q}}_{\mp}|\alpha,+2\rangle=\left|\bar{\chi}, \pm \frac{3}{2}\right\rangle
$$

and

$$
\begin{aligned}
& \overline{\mathcal{Q}}_{ \pm}|\alpha \pm d, \pm 1\rangle=\left|\bar{\chi}, \pm \frac{3}{2}\right\rangle, \quad \overline{\mathcal{Q}}_{\mp}|\alpha \pm d, \pm 1\rangle=0, \\
& \overline{\mathcal{Q}}_{\mp}|\alpha \mp d, \pm 1\rangle=\left|\bar{\chi}, \pm \frac{1}{2}\right\rangle, \quad \overline{\mathcal{Q}}_{ \pm}|\alpha \mp d, \pm 1\rangle=0 \text {, }
\end{aligned}
$$

and

$$
\overline{\mathcal{Q}}_{ \pm}|\alpha \pm d, 0\rangle=\left|\bar{\chi}, \pm \frac{1}{2}\right\rangle, \quad \overline{\mathcal{Q}}_{ \pm}|\alpha \mp d, 0\rangle=0
$$

The diamond is symmetric about the $j_{z}=0$ line. In other words, once we obtained all the transformations for the states in its upper half, the lower half can be filled up by interchanging momentum spinors $p \leftrightarrow q$. This holds by the construction of the massive helicity wavefunctions in appendix C, see also [1] and [10. 


\section{$7.2 \mathcal{N}=2$ supermultiplets}

The new feature of extended $\mathcal{N}=2$ SUSY is the non-Abelian $S U(2)$ R-symmetry group. The supercharges are spinors with respect to this $S U(2)$ and therefore carry fundamental indices $i$. That is why we have to introduce a bookkeeping Grassmann variable $\eta_{i}$ which decouples from the spacetime spinor index structure. In other words, this $\eta_{i}$ is a spinor of the R-symmetry but a scalar with respect to the spacetime $S O(1,3)$. We define supercharge components $\overline{\mathcal{Q}}_{ \pm}(\eta)$ which are associated with the choices $\bar{\eta}_{\dot{a}, i}^{+}=\eta_{i} p_{\dot{a}}^{*} /\langle p q\rangle$ and $\bar{\eta}_{\dot{a}, i}^{-}=\eta_{i} q_{\dot{a}}^{*} /\langle q p\rangle$ :

$$
\begin{aligned}
& \overline{\mathcal{Q}}_{+}(\eta)=\eta_{i} \frac{p_{\dot{a}}^{*}}{\langle p q\rangle} \overline{\mathcal{Q}}^{\dot{a}, i}, \\
& \overline{\mathcal{Q}}_{-}(\eta)=\eta_{i} \frac{q_{\dot{a}}^{*}}{\langle q p\rangle} \overline{\mathcal{Q}}^{\dot{a}, i} .
\end{aligned}
$$

In the construction of $\mathcal{N}=2$ supermultiplets from their Clifford vacua, we obtain states in nontrivial representations of the $S U(2)$ R-symmetry ${ }^{21}$ Their $S U(2)$ tensor structures will be displayed inside the ket vectors, right after the $J_{z}$ eigenvalue, separated by a semicolon, ${ }^{22}$

\subsubsection{Spin one supermultiplets}

Again, we start our presentation with the smaller multiplets of lower spin. The universal sector due to $\mathcal{N}=2$ SUSY encompasses two spin one multiplets with scalar Clifford vacua, see figures 10 and 11 below.

The first multiplet $\left\{\omega^{+}, a, r, \Phi^{-}, \zeta^{+}, \Omega_{A}^{+}\right\}$is constructed from a scalar Clifford vacuum $\zeta^{+}$, c.f. 6.73 . Omitting all the vanishing results, we obtain

$$
\overline{\mathcal{Q}}_{ \pm}\left(\eta_{i}\right)\left|\Phi^{+}, 0 ; 1\right\rangle=\left|\bar{a}, \pm \frac{1}{2} ; \eta_{i}\right\rangle
$$

and

$$
\begin{aligned}
& \overline{\mathcal{Q}}_{ \pm}\left(\epsilon_{j}\right)\left|\bar{a}, \pm \frac{1}{2}, \eta_{i}\right\rangle=\left|\omega^{-}, \pm 1 ;(\epsilon \eta)\right\rangle, \\
& \overline{\mathcal{Q}}_{\mp}\left(\epsilon_{j}\right)\left|\bar{a}, \pm \frac{1}{2}, \eta_{i}\right\rangle=\frac{1}{\sqrt{2}}\left|\omega^{-}, 0 ;(\epsilon \eta)\right\rangle \pm \frac{1}{\sqrt{2}}\left|\Omega^{-}, 0 ; \epsilon_{j}\left(\tau_{A} \varepsilon\right)^{j i} \eta_{i}\right\rangle
\end{aligned}
$$

\footnotetext{
${ }^{21}$ In fact, it is a peculiar feature of the first mass level that its Clifford vacua are R-symmetry scalars.

${ }^{22}$ In the literature, on-shell supersymmetry is usually described by the notion of supercharge eigenstates - Grassmann coherent states, firstly in [25, and recently in 24] and also [26]. Our presentation of SUSY transformations including internal wavefunctions (carrying the R-symmetry quantum numbers) are an equivalent way of expressing their information content.
} 


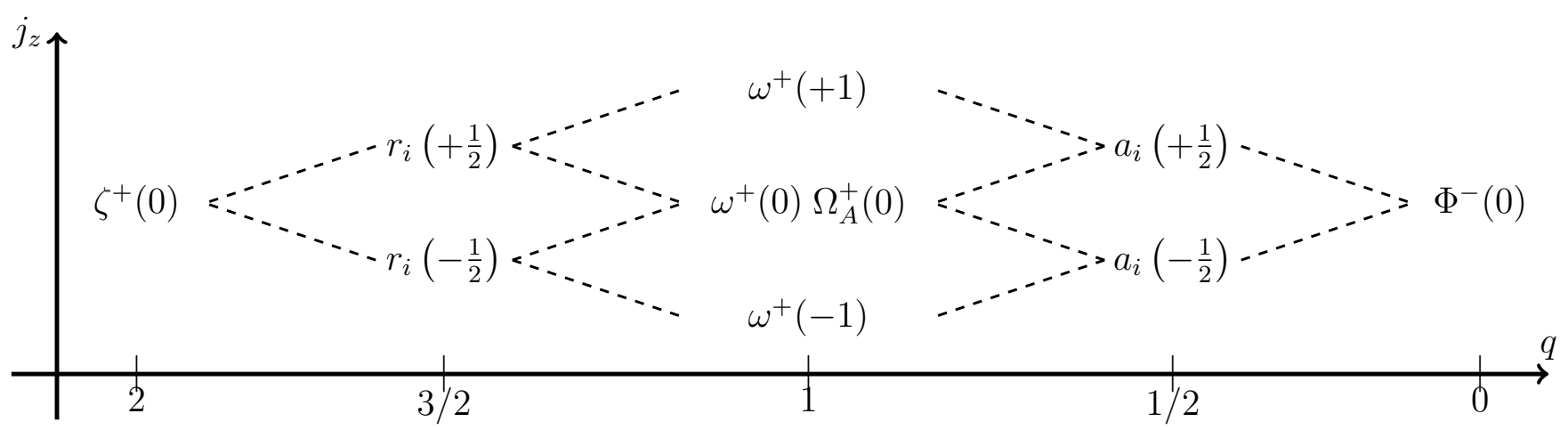

Figure 10: $\mathcal{N}=2$ SUSY multiplet with scalar Clifford vacuum: In $\mathcal{N}=2$ scenarios, the $U(1)$ charge $q$ with respect to the internal toroidal directions is plotted along the horizontal axis. Since the world-sheet fields $i \partial Z^{ \pm}$and $\mathrm{e}^{i q H}$ have charge \pm 1 and $q$, respectively, the SUSY generators built from $\mathrm{e}^{ \pm i H / 2}$ and $i \partial Z^{ \pm} \mathrm{e}^{\mp i H / 2}$ change $q$ by the fixed offset $\pm 1 / 2$.

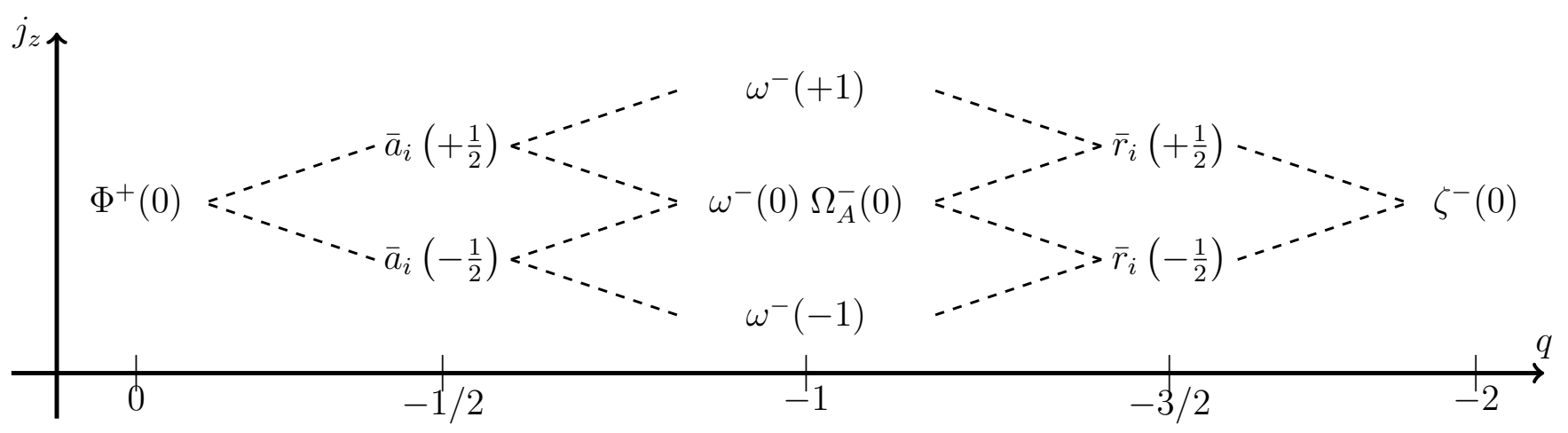

Figure 11: conjugate $\mathcal{N}=2$ SUSY multiplet with scalar Clifford vacuum

$$
\equiv\left|\omega^{-} \pm \Omega_{A}^{-}, 0\right\rangle,
$$

where $(\epsilon \eta)=\epsilon_{j} \varepsilon^{j i} \eta_{i}$. The $\omega^{-}$and $\Omega^{-}$states in the center of the diamond transform to

$$
\begin{gathered}
\overline{\mathcal{Q}}_{\mp}\left(\eta_{i}\right)\left|\omega^{-}, \pm 1 ;(\epsilon \eta)\right\rangle=\overline{\mathcal{Q}}_{ \pm}\left(\eta_{i}\right)\left|\omega^{-} \pm \Omega_{A}^{-}, 0\right\rangle=\left|\bar{r}, \pm \frac{1}{2} ;(\epsilon \eta) \eta_{i}\right\rangle, \\
\overline{\mathcal{Q}}_{\mp}\left(\eta_{i}\right)\left|\omega^{-} \pm \Omega_{A}^{-}, 0\right\rangle=0,
\end{gathered}
$$

and

$$
\overline{\mathcal{Q}}_{\mp}\left(\epsilon_{j}\right)\left|\bar{r}, \pm \frac{1}{2} ;(\epsilon \eta) \eta_{i}\right\rangle=\left|\zeta^{-}, 0 ;(\epsilon \eta)^{2}\right\rangle .
$$

Similar results are obtained for the mirror spin one multiplet $\left\{\omega^{-}, \bar{a}, \bar{r}, \Phi^{+}, \zeta^{-}, \Omega_{A}^{-}\right\}$, which is constructed from the scalar Clifford vacuum. The helicity SUSY transformations are

$$
\overline{\mathcal{Q}}_{ \pm}\left(\eta_{i}\right)\left|\zeta^{+}, 0 ; 1\right\rangle=\left|r, \pm \frac{1}{2} ; \eta_{i}\right\rangle,
$$


and

$$
\begin{aligned}
\overline{\mathcal{Q}}_{ \pm}\left(\epsilon_{j}\right)\left|r, \pm \frac{1}{2} ; \eta_{i}\right\rangle & =\left|\omega^{+}, \pm 1 ;(\epsilon \eta)\right\rangle, \\
\overline{\mathcal{Q}}_{\mp}\left(\epsilon_{j}\right)\left|r, \pm \frac{1}{2} ; \eta_{i}\right\rangle & =\frac{1}{\sqrt{2}}\left|\omega^{+}, 0 ;(\epsilon \eta)\right\rangle \pm \frac{1}{\sqrt{2}}\left|\Omega^{+}, 0 ; \epsilon_{j}\left(\tau_{A} \varepsilon\right)^{j i} \eta_{i}\right\rangle \\
& \equiv\left|\omega^{+} \pm \Omega^{+}, 0\right\rangle,
\end{aligned}
$$

and

$$
\begin{gathered}
\overline{\mathcal{Q}}_{\mp}\left(\eta_{i}\right)\left|\omega^{+}, \pm 1 ;(\epsilon \eta)\right\rangle=\overline{\mathcal{Q}}_{ \pm}\left(\eta_{i}\right)\left|\omega^{+} \pm \Omega^{+}, 0\right\rangle=\left|a, \pm \frac{1}{2} ;(\epsilon \eta) \eta_{i}\right\rangle, \\
\overline{\mathcal{Q}}_{\mp}\left(\eta_{i}\right)\left|\omega^{+} \pm \Omega^{+}, 0\right\rangle=0,
\end{gathered}
$$

and

$$
\overline{\mathcal{Q}}_{\mp}\left(\epsilon_{j}\right)\left|a, \pm \frac{1}{2} ;(\epsilon \eta) \eta_{i}\right\rangle=\left|\Phi^{-}, 0 ;(\epsilon \eta)^{2}\right\rangle .
$$

\subsubsection{Spin two supermultiplet}

The highest spin state of the first mass level populate a spin two multiplet $\left\{\alpha, \chi, \bar{\chi}, d, \xi, \beta^{ \pm}, s, \bar{s}, \phi\right\}$ (see figure 12), which is built from a vector Clifford vacuum $\beta_{\mu}^{+}$state, c.f. (6.39).

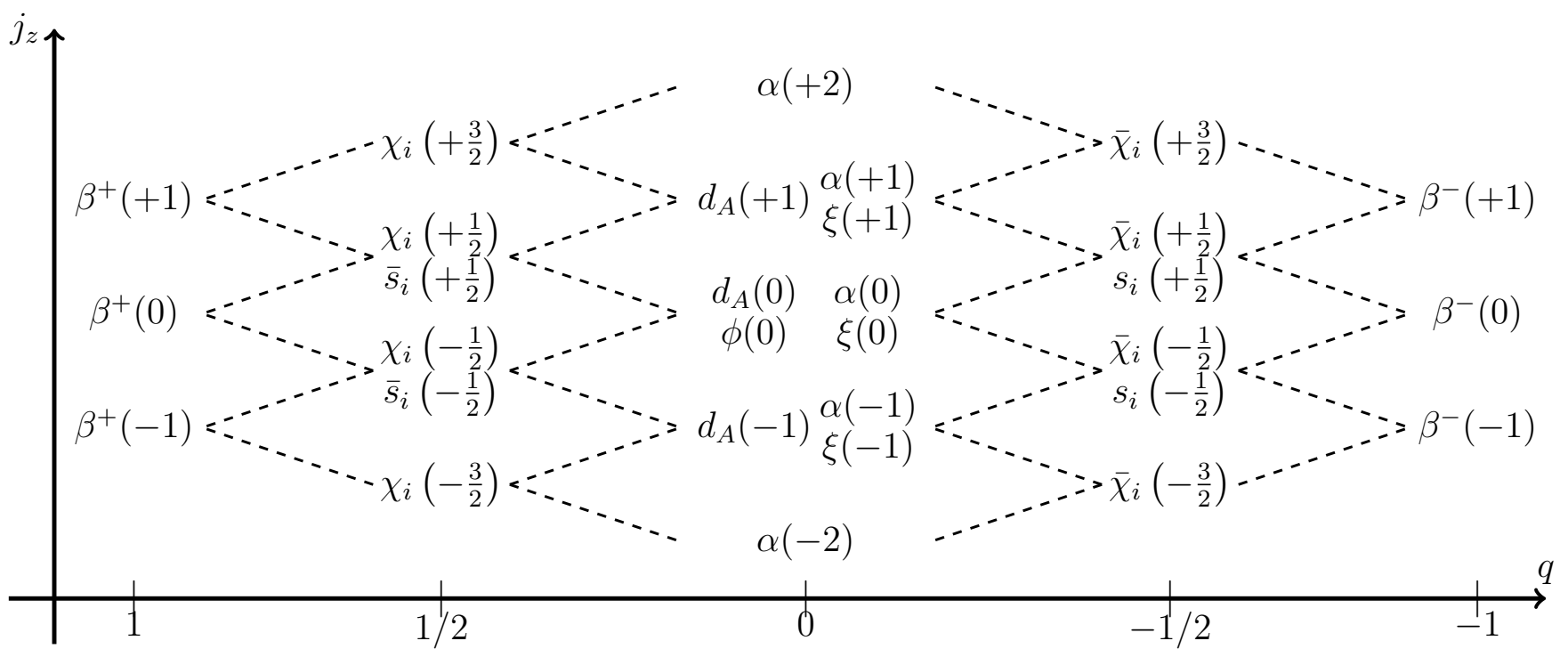

Figure 12: $\mathcal{N}=2$ SUSY multiplet with vector Clifford vacuum

The supermultiplet structure is more complicated here due to intersection points in the diamond like $\overline{\mathcal{Q}}_{-}\left(\eta_{i}\right)\left|\beta^{+},+1 ; 1\right\rangle \leftrightarrow \overline{\mathcal{Q}}_{+}\left(\eta_{i}\right)\left|\beta^{+}, 0 ; 1\right\rangle$. Since $j_{z} \mapsto-j_{z}$ reflection can be implemented by $p \leftrightarrow q$ 
exchange, we will only show the transformations for the upper half of the diamond. Omitting all the trivial relations, we obtain

$$
\begin{aligned}
\overline{\mathcal{Q}}_{+}\left(\eta_{i}\right)\left|\beta^{+},+1 ; 1\right\rangle & =\left|\chi,+\frac{3}{2} ; \eta_{i}\right\rangle, \\
\overline{\mathcal{Q}}_{-}\left(\eta_{i}\right)\left|\beta^{+},+1 ; 1\right\rangle & =\frac{1}{\sqrt{3}}\left|\chi,+\frac{1}{2} ; \eta_{i}\right\rangle+\frac{\sqrt{2}}{\sqrt{3}}\left|\bar{s},+\frac{1}{2} ; \eta_{i}\right\rangle \equiv\left|\chi \oplus \bar{s},+\frac{1}{2}\right\rangle_{1}, \\
\overline{\mathcal{Q}}_{+}\left(\eta_{i}\right)\left|\beta^{+}, 0 ; 1\right\rangle & =\frac{\sqrt{2}}{\sqrt{3}}\left|\chi,+\frac{1}{2} ; \eta_{i}\right\rangle-\frac{1}{\sqrt{3}}\left|\bar{s},+\frac{1}{2} ; \eta_{i}\right\rangle \equiv\left|\chi \oplus \bar{s},+\frac{1}{2}\right\rangle_{2},
\end{aligned}
$$

where $\left|\chi \oplus \bar{s},+\frac{1}{2}\right\rangle_{1}$ is orthogonal to $\left|\chi \oplus \bar{s},+\frac{1}{2}\right\rangle_{2}$. For the helicity SUSY transformation of the second column of figure 12 , we have

$$
\begin{aligned}
\overline{\mathcal{Q}}_{+}\left(\epsilon_{j}\right)\left|\chi,+\frac{3}{2} ; \eta_{i}\right\rangle & =|\alpha,+2 ;(\epsilon \eta)\rangle, \\
\overline{\mathcal{Q}}_{-}\left(\epsilon_{j}\right)\left|\chi,+\frac{3}{2} ; \eta_{i}\right\rangle & =-\frac{1}{2}|\alpha,+1 ;(\epsilon \eta)\rangle+\frac{1}{2}|\xi,+1 ;(\epsilon \eta)\rangle-\frac{1}{\sqrt{2}}|d,+1 ;(\epsilon \eta)\rangle \\
& \equiv|\alpha \oplus \xi \oplus d,+1\rangle_{1}, \\
\overline{\mathcal{Q}}_{+}\left(\epsilon_{j}\right)\left|\chi \oplus \bar{s},+\frac{1}{2}\right\rangle_{1} & =-\frac{1}{2}|\alpha,+1 ;(\epsilon \eta)\rangle+\frac{1}{2}|\xi,+1 ;(\epsilon \eta)\rangle+\frac{1}{\sqrt{2}}|d,+1 ;(\epsilon \eta)\rangle \\
& \equiv|\alpha \oplus \xi \oplus d,+1\rangle_{2}, \\
\overline{\mathcal{Q}}_{+}\left(\epsilon_{j}\right)\left|\chi \oplus \bar{s},+\frac{1}{2}\right\rangle_{2} & =-\frac{1}{\sqrt{2}}|\alpha,+1 ;(\epsilon \eta)\rangle-\frac{1}{\sqrt{2}}|\xi,+1 ;(\epsilon \eta)\rangle \\
& \equiv|\alpha \oplus \xi \oplus d,+1\rangle_{3} .
\end{aligned}
$$

One can easily check that the three states $|\alpha \oplus \xi \oplus d,+1\rangle_{1,2,3}$ are orthonormal. Moreover,

$$
\begin{aligned}
\overline{\mathcal{Q}}_{-}\left(\epsilon_{j}\right)\left|\chi \oplus \bar{s},+\frac{1}{2}\right\rangle_{1} & =-\frac{1}{\sqrt{6}}|\alpha, 0 ;(\epsilon \eta)\rangle+\frac{1}{\sqrt{2}}|\xi, 0 ;(\epsilon \eta)\rangle+\frac{1}{\sqrt{3}}|\phi, 0 ;(\epsilon \eta)\rangle \\
& \equiv|\alpha \oplus \xi \oplus d \oplus \phi\rangle_{1}, \\
\overline{\mathcal{Q}}_{-}\left(\epsilon_{j}\right)\left|\chi \oplus \bar{s},+\frac{1}{2}\right\rangle_{2} & =-\frac{1}{\sqrt{3}}|\alpha, 0 ;(\epsilon \eta)\rangle-\frac{1}{\sqrt{2}}|d, 0 ;(\epsilon \eta)\rangle-\frac{1}{\sqrt{6}}|\phi, 0 ;(\epsilon \eta)\rangle \\
& \equiv|\alpha \oplus \xi \oplus d \oplus \phi\rangle_{2} .
\end{aligned}
$$

By interchanging $p \leftrightarrow q$ we get the states

$|\alpha \oplus \xi \oplus d \oplus \phi\rangle_{1}^{\prime}=|\alpha \oplus \xi \oplus d \oplus \phi\rangle_{1}(p \leftrightarrow q)=-\frac{1}{\sqrt{6}}|\alpha, 0 ;(\epsilon \eta)\rangle-\frac{1}{\sqrt{2}}|\xi, 0 ;(\epsilon \eta)\rangle+\frac{1}{\sqrt{3}}|\phi, 0 ;(\epsilon \eta)\rangle$,

$|\alpha \oplus \xi \oplus d \oplus \phi\rangle_{2}^{\prime}=|\alpha \oplus \xi \oplus d \oplus \phi\rangle_{2}(p \leftrightarrow q)=-\frac{1}{\sqrt{3}}|\alpha, 0 ;(\epsilon \eta)\rangle+\frac{1}{\sqrt{2}}|d, 0 ;(\epsilon \eta)\rangle-\frac{1}{\sqrt{6}}|\phi, 0 ;(\epsilon \eta)\rangle$, 
which are the results obtained from $\overline{\mathcal{Q}}_{+}\left(\eta_{j}\right)\left|\chi \oplus \bar{s},-\frac{1}{2}\right\rangle$. Clearly, $|\alpha \oplus \xi \oplus d \oplus \phi\rangle_{1(2)}$ is orthogonal to $|\alpha \oplus \xi \oplus d \oplus \phi\rangle_{1(2)}^{\prime}$. The helicity SUSY transformations of this column are

$$
\begin{gathered}
\overline{\mathcal{Q}}_{-}\left(\eta_{i}\right)|\alpha,+2 ;(\epsilon \eta)\rangle=\overline{\mathcal{Q}}_{+}\left(\eta_{i}\right)|\alpha \oplus \xi \oplus d,+1\rangle_{1}=\left|\bar{\chi},+\frac{3}{2} ;(\epsilon \eta) \eta_{i}\right\rangle, \\
\overline{\mathcal{Q}}_{+}\left(\eta_{i}\right)|\alpha \oplus \xi \oplus d,+1\rangle_{2}=\overline{\mathcal{Q}}_{+}\left(\eta_{i}\right)|\alpha \oplus \xi \oplus d,+1\rangle_{3}=0,
\end{gathered}
$$

and

$$
\begin{aligned}
& \overline{\mathcal{Q}}_{-}\left(\eta_{i}\right)|\alpha \oplus \xi \oplus d,+1\rangle_{1}=0, \\
& \overline{\mathcal{Q}}_{-}\left(\eta_{i}\right)|\alpha \oplus \xi \oplus d,+1\rangle_{2}=\frac{1}{\sqrt{3}}\left|\bar{\chi},+\frac{1}{2} ;(\epsilon \eta) \eta_{i}\right\rangle-\frac{\sqrt{2}}{\sqrt{3}}\left|s,+\frac{1}{2} ;(\epsilon \eta) \eta_{i}\right\rangle \equiv\left|\bar{\chi} \oplus s,+\frac{1}{2}\right\rangle_{1}, \\
& \overline{\mathcal{Q}}_{-}\left(\eta_{i}\right)|\alpha \oplus \xi \oplus d,+1\rangle_{3}=\frac{\sqrt{2}}{\sqrt{3}}\left|\bar{\chi},+\frac{1}{2} ;(\epsilon \eta) \eta_{i}\right\rangle+\frac{1}{\sqrt{3}}\left|s,+\frac{1}{2} ;(\epsilon \eta) \eta_{i}\right\rangle \equiv\left|\bar{\chi} \oplus s,+\frac{1}{2}\right\rangle_{2} .
\end{aligned}
$$

States in the center of the diamond transform as

$$
\begin{gathered}
\overline{\mathcal{Q}}_{+}\left(\eta_{i}\right)|\alpha \oplus \xi \oplus d \oplus \phi\rangle_{1}^{\prime}=\overline{\mathcal{Q}}_{+}\left(\eta_{i}\right)|\alpha \oplus \xi \oplus d \oplus \phi\rangle_{2}^{\prime}=0, \\
\overline{\mathcal{Q}}_{+}\left(\eta_{i}\right)|\alpha \oplus \xi \oplus d \oplus \phi\rangle_{1}=\left|\bar{\chi} \oplus s,+\frac{1}{2}\right\rangle_{1}, \\
\overline{\mathcal{Q}}_{+}\left(\eta_{i}\right)|\alpha \oplus \xi \oplus d \oplus \phi\rangle_{2}=\left|\bar{\chi} \oplus s,+\frac{1}{2}\right\rangle_{2},
\end{gathered}
$$

where $\left|\bar{\chi} \oplus s,+\frac{1}{2}\right\rangle_{1}$ and $\left|\bar{\chi} \oplus s,+\frac{1}{2}\right\rangle_{2}$ are orthogonal to each other. Now we are left with the transformations to the anti-Clifford vacuum states $\left|\beta^{-}\right\rangle$in last column of the diamond:

$$
\begin{gathered}
\overline{\mathcal{Q}}_{-}\left(\epsilon_{j}\right)\left|\bar{\chi},+\frac{3}{2} ;(\epsilon \eta) \eta_{i}\right\rangle=\overline{\mathcal{Q}}_{+}\left(\epsilon_{j}\right)\left|\bar{\chi} \oplus s,+\frac{1}{2}\right\rangle_{1}=\left|\beta^{-},+1 ;(\epsilon \eta)^{2}\right\rangle, \\
\overline{\mathcal{Q}}_{+}\left(\epsilon_{j}\right)\left|\bar{\chi} \oplus s,+\frac{1}{2}\right\rangle_{2}=\overline{\mathcal{Q}}_{-}\left(\epsilon_{j}\right)\left|\bar{\chi} \oplus s,+\frac{1}{2}\right\rangle_{1}=0, \\
\overline{\mathcal{Q}}_{-}\left(\epsilon_{j}\right)\left|\bar{\chi} \oplus s,+\frac{1}{2}\right\rangle_{2}=\left|\beta^{-}, 0 ;(\epsilon \eta)^{2}\right\rangle .
\end{gathered}
$$

This completes the helicity SUSY transformations for the upper half of the diamond representing the spin two supermultiplet of $\mathcal{N}=2$.

\section{3 $\mathcal{N}=4$ supermultiplet}

In $\mathcal{N}=4$ SUSY, the supercharges carry internal $S O(6) \equiv S U(4)$ spinor indices $I$ or $\bar{I}$. Similar to $\mathcal{N}=2$ case, we introduce the internal spinors $\eta_{I}$ and $\bar{\eta}^{\bar{I}}$. Then the components of the (righthanded) anti-supercharge can be written as

$$
\overline{\mathcal{Q}}_{+}=\bar{\eta}^{\bar{I}} \frac{p_{\dot{a}}^{*}}{\langle p q\rangle} \overline{\mathcal{Q}}_{\bar{I}}^{\dot{a}}, \quad \overline{\mathcal{Q}}_{-}=\bar{\eta}^{\bar{I}} \frac{q_{\dot{a}}^{*}}{\langle q p\rangle} \overline{\mathcal{Q}}_{\bar{I}}^{\dot{a}} .
$$


We only have one big spin two supermultiplet in $\mathcal{N}=4$, see figure 13. Starting from the Clifford vacuum $\Phi^{+}$, c.f. 4.40 , the remainder of the multiplet is filled by $\overline{\mathcal{Q}}_{ \pm}$application. Following the symmetry argument of the last subsections, we will only show the helicity SUSY transformation of the states in the upper half $j_{z} \geq 0$ of the diamond. And again, the internal wavefunctions of the physical states are displayed right behind the semicolon in the ket.

We start from Clifford vacuum state $\left|\Phi^{+}, 0 ; 1\right\rangle$ located at the far left of the diamond. The helicity SUSY transformations read

$$
\overline{\mathcal{Q}}_{+}\left(\bar{\eta}^{\bar{I}}\right)\left|\Phi^{+}, 0 ; 1\right\rangle=\left|\bar{a},+\frac{1}{2} ; \bar{\eta}^{\bar{I}}\right\rangle
$$

and

$$
\begin{aligned}
\overline{\mathcal{Q}}_{+}\left(\bar{\epsilon}^{\bar{J}}\right)\left|\bar{a},+\frac{1}{2} ; \bar{\eta}^{\bar{I}}\right\rangle & =\left|\beta^{-},+1 ; \frac{1}{\sqrt{2}} \bar{\epsilon}^{\bar{J}}\left(\bar{\gamma}_{m} C\right)_{\bar{J} \bar{I}} \bar{\eta}^{\bar{I}}\right\rangle, \\
\overline{\mathcal{Q}}_{-}\left(\bar{\epsilon}^{\bar{J}}\right)\left|\bar{a},+\frac{1}{2} ; \bar{\eta}^{\bar{I}}\right\rangle & =-\frac{1}{\sqrt{2}}\left|\beta^{-}, 0 ; \frac{1}{\sqrt{2}} \bar{\epsilon}^{\bar{J}}\left(\bar{\gamma}_{m} C\right)_{\bar{J} \bar{I}} \bar{\eta}^{\bar{I}}\right\rangle+\frac{1}{\sqrt{2}}\left|\Omega^{-}, 0 ; \frac{1}{12} \bar{\epsilon}^{\bar{J}}\left(\bar{\gamma}_{m n l} C\right)_{\bar{J} \bar{I}} \bar{\eta}^{\bar{I}}\right\rangle \\
& \equiv\left|\beta^{-} \oplus \Omega^{-}, 0\right\rangle,
\end{aligned}
$$

and

$$
\begin{aligned}
& \overline{\mathcal{Q}}_{+}\left(\bar{\xi}^{\bar{K}}\right)\left|\beta^{-},+1 ; \frac{1}{\sqrt{2}} \bar{\epsilon}^{\bar{J}}\left(\bar{\gamma}_{m} C\right)_{\bar{J} \bar{I}} \bar{\eta}^{\bar{I}}\right\rangle=\left|\chi,+\frac{3}{2} ; \varepsilon_{\bar{I} \bar{J} \bar{K} \bar{L}} \bar{\eta}^{\bar{I}} \bar{\epsilon}^{\bar{J}} \bar{\xi}^{\bar{K}} C_{L}^{\bar{L}}\right\rangle, \\
& \overline{\mathcal{Q}}_{-}\left(\bar{\xi}^{\bar{K}}\right)\left|\beta^{-},+1 ; \frac{1}{\sqrt{2}} \bar{\epsilon}^{\bar{J}}\left(\bar{\gamma}_{m} C\right)_{\bar{J} \bar{I}} \bar{\eta}^{\bar{I}}\right\rangle=-\frac{1}{\sqrt{3}}\left|\chi,+\frac{1}{2} ; \varepsilon_{\bar{I} \bar{J} \bar{K} \bar{L}} \bar{\eta}^{\bar{I}} \bar{\epsilon}^{\bar{J}} \bar{\xi}^{\bar{K}} C_{L}^{\bar{L}}\right\rangle+\frac{\sqrt{2}}{\sqrt{3}}\left|\bar{r},+\frac{1}{2} ; \bar{r}_{\beta}\right\rangle \\
& \equiv\left|\chi \oplus \bar{r},+\frac{1}{2}\right\rangle_{1} \\
& \overline{\mathcal{Q}}_{+}\left(\bar{\xi}^{\bar{K}}\right)\left|\beta^{-} \oplus \Omega^{-}, 0\right\rangle=\frac{1}{\sqrt{3}}\left|\chi,+\frac{1}{2} ; \varepsilon_{\bar{I}} \bar{J} \bar{K} \bar{L} \bar{\eta}^{\bar{I}} \bar{\epsilon}^{\bar{J}} \bar{\xi}^{\bar{K}} C_{L}^{\bar{L}}\right\rangle+\frac{1}{\sqrt{6}}\left|\bar{r},+\frac{1}{2} ; \bar{r}_{\beta}\right\rangle+\frac{1}{\sqrt{2}}\left|\bar{r},+\frac{1}{2} ; \bar{r}_{\Omega}\right\rangle \\
& \equiv\left|\chi \oplus \bar{r},+\frac{1}{2}\right\rangle_{2}
\end{aligned}
$$

where

$$
\begin{aligned}
& \bar{r}_{\beta}=\frac{\sqrt{3}}{2} \bar{\epsilon}^{\bar{J}}\left(\bar{\gamma}^{m} C\right)_{\bar{J} \bar{I}} \bar{\eta}^{\bar{I}} \bar{\xi}^{\bar{K}}\left(\delta_{m n}^{(6)} \delta_{\bar{K}}{ }^{\bar{L}}+\frac{1}{6}\left(\bar{\gamma}_{m} \gamma_{n}\right)_{\bar{K}}{ }^{\bar{L}}\right), \\
& \bar{r}_{\Omega}=\frac{1}{48} \bar{\epsilon}^{\bar{J}}\left(\bar{\gamma}_{m n l} C\right)_{\bar{J} \bar{I}} \bar{\eta}^{\bar{I}} \bar{\xi}^{\bar{K}}\left(\bar{\gamma}_{k} \gamma^{m n l}\right)_{\bar{K}}{ }^{\bar{L}} .
\end{aligned}
$$

Note that $\bar{r}_{\beta}$ and $\bar{r}_{\Omega}$ represent different and mutually orthogonal internal wavefunctions of $\bar{r}$.

The left-handed spin $3 / 2$ states in the third column of the $\mathcal{N}=4$ diamond transform to

$\overline{\mathcal{Q}}_{+}\left(\bar{\theta}^{\bar{M}}\right)\left|\chi,+\frac{3}{2} ; \varepsilon_{\bar{I} \bar{J} \bar{K} \bar{L}} \bar{\eta}^{\bar{I}} \bar{\epsilon}^{\bar{J}} \bar{\xi}^{\bar{K}} C_{L}^{\bar{L}}\right\rangle=|\alpha,+2 ; \varepsilon(\bar{\eta} \bar{\epsilon} \bar{\xi} \bar{\theta})\rangle$, 


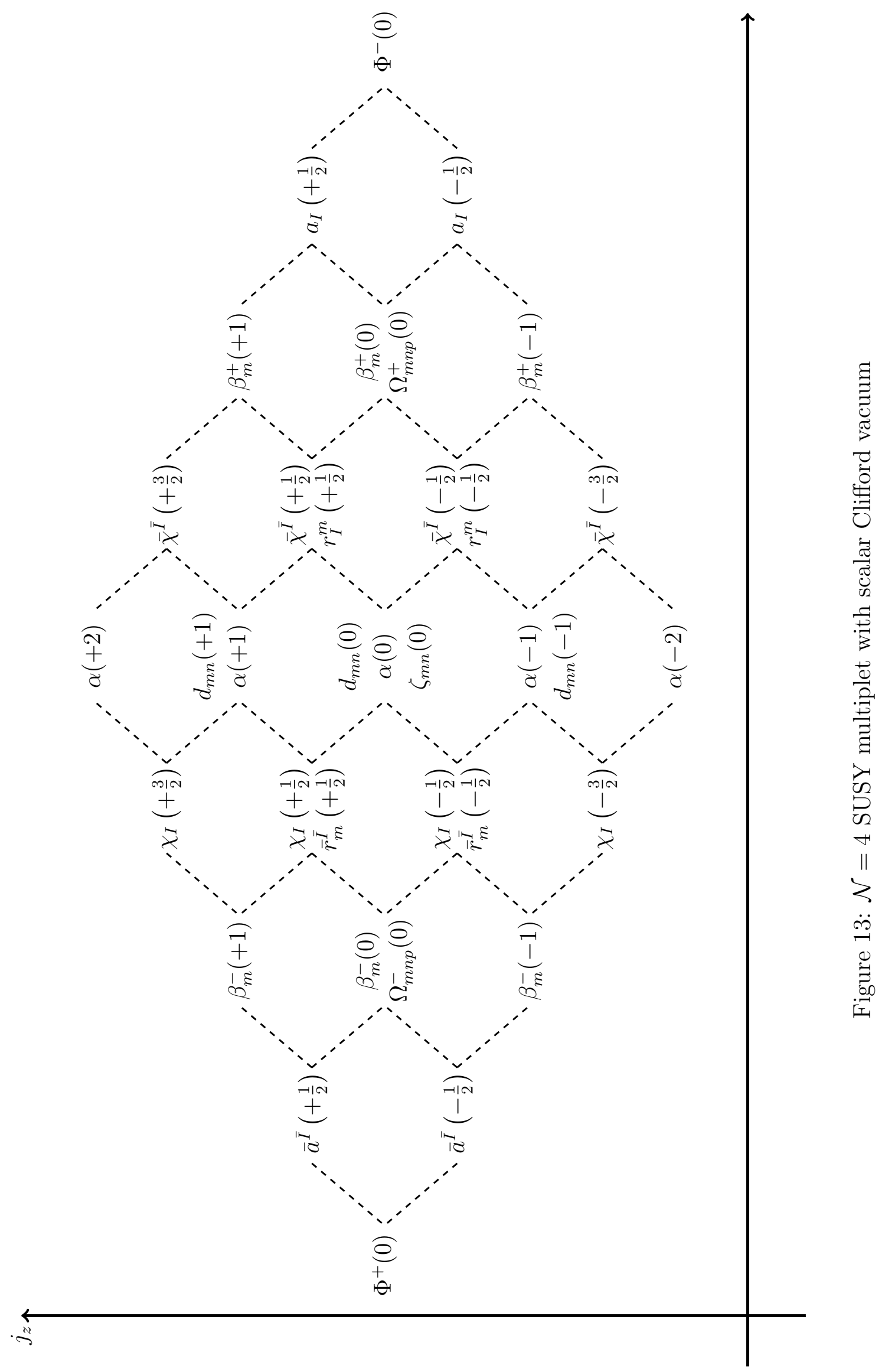




$$
\begin{aligned}
\overline{\mathcal{Q}}_{-}\left(\bar{\theta}^{\bar{M}}\right)\left|\chi,+\frac{1}{2} ; \varepsilon_{\bar{I} \bar{J} \bar{K} \bar{L}} \bar{\eta}^{\bar{I}} \bar{\epsilon}^{\bar{J}} \bar{\xi}^{\bar{K}} C_{L}^{\bar{L}}\right\rangle & =-\frac{1}{2}|\alpha,+1 ; \varepsilon(\bar{\eta} \bar{\epsilon} \bar{\xi} \bar{\theta})\rangle+\frac{\sqrt{3}}{2}\left|d,+1 ; d_{\chi}\right\rangle \equiv|\alpha \oplus d,+1\rangle_{1} \\
\overline{\mathcal{Q}}_{+}\left(\bar{\theta}^{\bar{M}}\right)\left|\chi \oplus \bar{r},+\frac{1}{2}\right\rangle_{1} & =-\frac{1}{2}|\alpha,+1 ; \varepsilon(\bar{\eta} \bar{\epsilon} \bar{\xi} \bar{\theta})\rangle-\frac{1}{2 \sqrt{3}}\left|d,+1 ; d_{\chi}\right\rangle+\frac{\sqrt{2}}{\sqrt{3}}\left|d,+1 ; d_{\bar{r}_{\beta}}\right\rangle \\
& \equiv|\alpha \oplus d,+1\rangle_{2}, \\
\overline{\mathcal{Q}}_{+}\left(\bar{\theta}^{\bar{M}}\right)\left|\chi \oplus \bar{r},+\frac{1}{2}\right\rangle_{2} & =\frac{1}{2}|\alpha,+1 ; \varepsilon(\bar{\eta} \bar{\epsilon} \bar{\xi} \bar{\theta})\rangle+\frac{1}{2 \sqrt{3}}\left|d,+1 ; d_{\chi}\right\rangle+\frac{1}{\sqrt{6}}\left|d,+1 ; d_{\bar{r}_{\beta}}\right\rangle \\
& +\frac{1}{\sqrt{2}}\left|d,+1 ; d_{\bar{r}_{\Omega}}\right\rangle \equiv|\alpha \oplus d,+1\rangle_{3}, \\
\overline{\mathcal{Q}}_{-}\left(\bar{\theta}^{\bar{M}}\right)\left|\chi \oplus \bar{r},+\frac{1}{2}\right\rangle_{1} & =\frac{1}{\sqrt{6}}|\alpha, 0 ; \varepsilon(\bar{\eta} \bar{\epsilon} \bar{\xi} \bar{\theta})\rangle-\frac{1}{\sqrt{6}}\left|d, 0 ; d_{\chi}\right\rangle+\frac{1}{\sqrt{3}}\left|d, 0 ; d_{\bar{r}_{\beta}}\right\rangle+\frac{1}{\sqrt{3}}\left|\zeta, 0 ; \zeta_{\bar{r}_{\beta}}\right\rangle \\
& \equiv|\alpha \oplus d \oplus \zeta, 0\rangle_{1}, \\
\overline{\mathcal{Q}}_{-}\left(\bar{\theta}^{\bar{M}}\right)\left|\chi \oplus \bar{r},+\frac{1}{2}\right\rangle_{2} & =-\frac{1}{\sqrt{6}}|\alpha, 0 ; \varepsilon(\bar{\eta} \bar{\epsilon} \bar{\xi} \bar{\theta})\rangle+\frac{1}{\sqrt{6}}\left|d, 0 ; d_{\chi}\right\rangle+\frac{1}{2 \sqrt{3}}\left|d, 0 ; d_{\bar{r}_{\beta}}\right\rangle \\
& +\frac{1}{2}\left|d, 0 ; d_{\bar{r}_{\Omega}}\right\rangle+\frac{1}{2 \sqrt{3}}\left|\zeta, 0 ; \zeta_{\bar{r}_{\beta}}\right\rangle+\frac{1}{2}\left|\zeta, 0 ; \zeta_{\bar{r}_{\Omega}}\right\rangle \\
& \equiv|\alpha \oplus d \oplus \zeta, 0\rangle_{2},
\end{aligned}
$$

where we have used the following abbreviations:

$$
\begin{aligned}
& \varepsilon(\bar{\eta} \bar{\epsilon} \bar{\xi} \bar{\theta})=\varepsilon_{\bar{I} \bar{J} \bar{K} \bar{L}} \bar{\eta}^{\bar{I}} \bar{\epsilon}^{\bar{J}} \bar{\xi}^{\bar{K}} \bar{\theta}^{\bar{L}}, \\
& d_{\chi}=\frac{1}{2 \sqrt{3}} \bar{\theta}^{\bar{M}}\left(\bar{\gamma}^{[m} \gamma^{n]}\right)_{\bar{M}}{ }^{\bar{L}} \varepsilon_{\bar{I} \bar{J} \bar{K} \bar{L}} \bar{\eta}^{\bar{I}} \bar{\epsilon}^{\bar{J}} \bar{\xi}^{\bar{K}}, \\
& d_{\bar{r}_{\beta}}=\frac{1}{\sqrt{2}} \bar{\theta}^{\bar{M}} \bar{r}_{\beta}^{[m|, \bar{L}|}\left(\bar{\gamma}^{n]} C\right)_{\bar{M} \bar{L}}, \quad d_{\bar{r}_{\Omega}}=\frac{1}{\sqrt{2}} \bar{\theta}^{\bar{M}} \bar{r}_{\Omega}^{[m|, \bar{L}|}\left(\bar{\gamma}^{n]} C\right)_{\bar{M} \bar{L}}, \\
& \zeta_{\bar{r}_{\beta}}=\frac{1}{\sqrt{2}} \bar{\theta}^{\bar{M}} \bar{r}_{\beta}^{(m|, \bar{L}|}\left(\bar{\gamma}^{n)} C\right)_{\bar{M} \bar{L}}, \quad \zeta_{\bar{r}_{\Omega}}=\frac{1}{\sqrt{2}} \bar{\theta}^{\bar{M}} \bar{r}_{\Omega}^{(m|, \bar{L}|}\left(\bar{\gamma}^{n)} C\right)_{\bar{M} \bar{L}}
\end{aligned}
$$

Similarly, $d_{\chi}, d_{\bar{r}_{\beta}}, d_{\bar{r}_{\Omega}}$ and $\zeta_{\bar{r}_{\beta}}, \zeta_{\bar{r}_{\Omega}}$ are two pairs of orthogonal states with respect to the internal R-symmetry. Thus, the explicit computation confirms that different states located at the same point inside the diamond (with the same $j_{z}$ ) are orthogonal to each other.

Now we are left with the helicity SUSY transformations for the right half of the diamond. After some manipulations, we obtain

$$
\begin{aligned}
& \overline{\mathcal{Q}}_{-}\left(\bar{\eta}^{\bar{I}}\right)|\alpha,+2 ; \varepsilon(\bar{\eta} \bar{\epsilon} \bar{\xi} \bar{\theta})\rangle=\overline{\mathcal{Q}}_{+}\left(\bar{\eta}^{\bar{I}}\right)|\alpha \oplus d,+1\rangle_{1}=\left|\bar{\chi},+\frac{3}{2} ; \varepsilon(\bar{\eta} \bar{\epsilon} \bar{\xi} \bar{\theta}) \bar{\eta}^{\bar{I}}\right\rangle, \\
& \overline{\mathcal{Q}}_{-}\left(\bar{\eta}^{\bar{I}}\right)|\alpha \oplus d,+1\rangle_{2}=\overline{\mathcal{Q}}_{+}\left(\bar{\eta}^{\bar{I}}\right)|\alpha \oplus d \oplus \zeta, 0\rangle_{1} \\
& \quad=\frac{1}{\sqrt{3}}\left|\bar{\chi},+\frac{1}{2} ; \varepsilon(\bar{\eta} \bar{\epsilon} \bar{\xi} \bar{\theta}) \bar{\eta}^{\bar{I}}\right\rangle-\frac{\sqrt{2}}{\sqrt{3}}\left|r,+\frac{1}{2} ; \varepsilon(\bar{\eta} \bar{\epsilon} \bar{\xi} \bar{\theta}) \bar{\eta}^{\bar{I}} \bar{\gamma}_{\bar{I} I}^{m}\left(\delta_{m n}^{(6)} \delta_{J}^{I}+\left(\gamma_{m} \bar{\gamma}_{n}\right)_{J}^{I}\right)\right\rangle
\end{aligned}
$$




$$
\begin{aligned}
& \equiv\left|\bar{\chi} \oplus r,+\frac{1}{2}\right\rangle_{1}, \\
& \overline{\mathcal{Q}}_{-}\left(\bar{\eta}^{\bar{I}}\right)|\alpha \oplus d,+1\rangle_{3}=\overline{\mathcal{Q}}_{+}\left(\bar{\eta}^{\bar{I}}\right)|\alpha \oplus d \oplus \zeta, 0\rangle_{2} \\
&=\frac{1}{\sqrt{3}}\left|\bar{\chi},+\frac{1}{2} ; \varepsilon(\bar{\eta} \bar{\epsilon} \bar{\xi} \bar{\theta}) \bar{\eta}^{\bar{I}}\right\rangle+\frac{1}{\sqrt{6}}\left|r,+\frac{1}{2} ; \varepsilon(\bar{\eta} \bar{\epsilon} \bar{\xi} \bar{\theta}) \bar{\eta}^{\bar{I}} \bar{\gamma}_{\bar{I} I}^{m}\left(\delta_{m n}^{(6)} \delta_{J}^{I}+\left(\gamma_{m} \bar{\gamma}_{n}\right)_{J}^{I}\right)\right\rangle \\
& \quad+\frac{1}{\sqrt{2}}\left|r,+\frac{1}{2} ; \varepsilon(\bar{\eta} \bar{\epsilon} \bar{\xi} \bar{\theta}) \bar{\eta}^{\bar{I}} \bar{\gamma}_{\bar{I} I}^{m n l}\left(\gamma_{k} \bar{\gamma}_{m n l}\right)_{J}^{I}\right\rangle \\
& \equiv\left|\bar{\chi} \oplus r,+\frac{1}{2}\right\rangle_{2},
\end{aligned}
$$

and

$$
\begin{aligned}
\overline{\mathcal{Q}}_{-}\left(\bar{\epsilon}^{\bar{J}}\right)\left|\bar{\chi},+\frac{3}{2} ; \varepsilon(\bar{\eta} \bar{\epsilon} \bar{\xi} \bar{\theta}) \bar{\eta}^{\bar{I}}\right\rangle= & \overline{\mathcal{Q}}_{+}\left(\bar{\epsilon}^{\bar{J}}\right)\left|\bar{\chi} \oplus r,+\frac{1}{2}\right\rangle_{1}=\left|\beta^{+},+1 ; \frac{1}{\sqrt{2}} \varepsilon(\bar{\eta} \bar{\epsilon} \bar{\xi} \bar{\theta}) \bar{\epsilon}^{\bar{J}}\left(\bar{\gamma}_{m} C\right)_{\bar{J} \bar{I}} \bar{\eta}^{\bar{I}}\right\rangle, \\
\overline{\mathcal{Q}}_{-}\left(\bar{\epsilon}^{\bar{J}}\right)\left|\bar{\chi} \oplus r,+\frac{1}{2}\right\rangle_{2}= & -\frac{1}{\sqrt{2}}\left|\beta^{+}, 0 ; \frac{1}{\sqrt{2}} \varepsilon(\bar{\eta} \bar{\epsilon} \bar{\xi} \bar{\theta}) \bar{\epsilon}^{\bar{J}}\left(\bar{\gamma}_{m} C\right)_{\bar{J} \bar{I}} \bar{\eta}^{\bar{I}}\right\rangle \\
& +\frac{1}{\sqrt{2}}\left|\Omega^{+}, 0 ; \frac{1}{12} \varepsilon(\bar{\eta} \bar{\epsilon} \bar{\xi} \bar{\theta}) \bar{\epsilon}^{\bar{J}}\left(C \gamma_{m n l}\right)_{\bar{J} \bar{I}} \bar{\eta} \bar{I}\right\rangle \equiv\left|\beta^{+} \oplus \Omega^{+}, 0\right\rangle,
\end{aligned}
$$

and

$\overline{\mathcal{Q}}_{-}\left(\bar{\xi}^{\bar{K}}\right)\left|\beta^{+},+1 ; \frac{1}{\sqrt{2}} \varepsilon(\bar{\eta} \bar{\epsilon} \bar{\xi} \bar{\theta}) \bar{\epsilon}^{\bar{J}}\left(\bar{\gamma}_{m} C\right)_{\bar{J} \bar{I}} \bar{\eta}^{\bar{I}}\right\rangle=\overline{\mathcal{Q}}_{+}\left(\bar{\xi}^{\bar{K}}\right)\left|\beta^{+} \oplus \Omega^{+}, 0\right\rangle=\left|a,+\frac{1}{2} ; \varepsilon(\bar{\eta} \bar{\epsilon} \bar{\xi} \bar{\theta}) \varepsilon_{\bar{I} \bar{J} \bar{K} \bar{L}} \bar{\eta}^{\bar{I}} \bar{\epsilon}^{\bar{J}} \bar{\xi}^{\bar{K}} C_{L}^{\bar{L}}\right\rangle$,

and finally we have

$$
\overline{\mathcal{Q}}_{-}(\bar{\theta} \bar{L})\left|a,+\frac{1}{2} ; \varepsilon(\bar{\eta} \bar{\epsilon} \bar{\xi} \bar{\theta}) \varepsilon_{\bar{I} \bar{J} \bar{K} \bar{L}} \bar{\eta}^{\bar{I}} \bar{\epsilon}^{\bar{J}} \bar{\xi}^{\bar{K}} C_{L}^{\bar{L}}\right\rangle=\left|\Phi^{+}, 0 ;[\varepsilon(\bar{\eta} \bar{\epsilon} \bar{\xi} \bar{\theta})]^{2}\right\rangle
$$

This completes the chain of transformations that take the Clifford vacuum $\left|\Phi^{+}\right\rangle$into its anti-Clifford counterpart $\left|\Phi^{-}\right\rangle$.

\section{Conclusions \& Outlook}

The main purpose of this paper is the explicit construction of vertex operators and SUSY transformation of universal multiplets of the first mass level. In sections 4, 5 and 6, we have identified the $\alpha^{\prime} m^{2}=1$ particle content of superstring compactifications to four dimensions whose presence is implied by $\mathcal{N}=4,1,2$ SUSY respectively. The universality arguments are based on Ramond sector SCFT operators which necessarily enter the SUSY charges and are available for building vertex operators, see section 3 and [12, 13, 14]. Then, using subleading terms of OPEs, we have explicitly evaluated all the SUSY transformations and found that the 24 (80) first mass level states 
in $\mathcal{N}=1(\mathcal{N}=2)$ scenarios are aligned into three supermultiplets. This has to be contrasted with the maximally supersymmetric case where the 256 states form one single $\mathcal{N}=4$ multiplet.

The multiplet structure of all the cases is investigated using spinor helicity methods and the results are summarized in figures 8,9 for $\mathcal{N}=1$, figures 10,11 , 12 for $\mathcal{N}=2$ and figure 13 for $\mathcal{N}=4$. We worked out the transformation properties of helicity eigenstates along a covariantly chosen quantization axis, see section 7 for the main results and appendix C for some background information on spinor helicity methods.

This work motivates a lot of further studies. It would be desirable to determine the universal particle content at higher mass levels, i.e. to classify the $S O(3)$ and R-symmetry quantum numbers of universal $\mathcal{N}=1$ and $\mathcal{N}=2$ SUSY multiplets of any mass level along the lines of the $S O(9)$ analysis in [27]. Explicit vertex operators on the second mass level are available in ten [8] and four dimensions [10]. These results suggest an investigation of subleading Regge trajectories, i.e. closed form expressions for vertex operators of non-maximal spin $n, n-1, \ldots$ at mass level $n$.

Having a good control over vertex operators is necessary to gain further insight into the $\mathrm{S}$ matrix of massive string excitations. The leading Regge trajectory is a good example where cubic and quartic interactions could be discussed for all mass levels, see [7] for bosonic string theory and [28] for the superstring. The simple structure of the $N$-point open superstring disk amplitude of massless states [29, 30] suggests that also the amplitudes of heavy vibration modes enjoy a hidden harmony. It would be desirable to work out the kinematic building blocks and the most natural basis of world-sheet integrals for their tree- and loop amplitudes.

Supersymmetry is certainly a key ingredient for investigating scattering amplitudes of massive states. An efficient way of constraining (or in some cases even determining) massive superamplitudes via supersymmetric Ward identities is explained in [31, 32]. For the purpose of a full-fledged superstring computation, the pure spinor formalism [33] is a very useful approach to take advantage of manifest supersymmetry. Unfortunately, the only explicitly known vertex operators in pure spinor superspace are at mass levels zero and one [6, 9], so determining and applying their higher mass counterparts is an open challenge. Also, there exists a manifestly $\mathcal{N}=1$ supersymmetric approach in four dimensions known as the hybrid formalism [34], see [35, 36] for a treatment of the first mass level in this framework. In any case, understanding the super Poincaré multiplet structure of massive states is the indispensable first step to exploit the power of SUSY for scattering amplitude, this was a key motivation for the present article. 


\section{Acknowledgments}

This work is supported by the DFG Transregional Collaborative Research Centre TRR 33 and the DFG cluster of excellence "Origin and Structure of the Universe". WZ.F. is also supported in part by the National Science Foundation Grant PHY-0757959. Any opinions, findings, and conclusions or recommendations expressed in this material are those of the author and do not necessarily reflect the views of the National Science Foundation. WZ.F. is grateful to the MaxPlanck-Institut für Physik (Werner-Heisenberg-Institut) for its kind hospitality while this work was initiated. He also would like to thank Cheng Peng for very helpful discussions at the final stage of this work. D.L. likes to thank the Simons Center for Geometry and Physics for its hospitality. O.S. would like to thank Northeastern University for hospitality during the time of preparation and Rutger Boels, Stefan Hohenegger, Stephan Stieberger and Tomasz Taylor for enlightening discussions. We are also very grateful to Robert Richter for carefully reading our draft and giving us multiple valuable suggestions.

\section{A Notation and convention}

Various types of indices appear in this article, so it is essential to keep the notation as clear and unambiguous as possible. Here is a list of occurring index classes together with the preferably used alphabets and letters:

- In ten dimensions, vector indices of $S O(1,9)$ are taken from the middle of the Latin alphabet $m, n, p, \ldots$ The corresponding Weyl spinor indices are Greek letters from the beginning of the alphabet, $\alpha, \beta, \gamma, \ldots$ for left-handed spinors, and their dotted version $\dot{\alpha}, \dot{\beta}, \dot{\gamma}, \ldots$ for the right-handed counterparts.

- Vectors in four-dimensional Minkowski spacetime have indices from the middle of the Greek alphabet $\mu, \nu, \lambda, \rho, \ldots$. Spinor indices of $S O(1,3)$ are lower case Latin letters $a, b, c, \ldots$ for left-handed Weyl spinors and upper case $\dot{a}, \dot{b}, \dot{c}$ for right-handed Weyl spinors.

- The R-symmetry group of $\mathcal{N}=4$ spacetime SUSY is $S O(6) \equiv S U(4)$. We will use $m, n, p \ldots$ as vector indices and $I, J, K(\bar{I}, \bar{J}, \bar{K})$ as left-handed (right-handed) spinor indices. Confusions with the $D=10$ vector indices are excluded by the context.

- In case of $\mathcal{N}=2$ spacetime SUSY, we denote the fundamental indices of the $S U(2) \mathrm{R}$ symmetry by $i, j, k$ and the corresponding adjoint indices by $A, B, C$. 
- Chan-Paton generators carrying the color degrees of freedom of the vertex operator are suppressed throughout this work since they are the same for all members of the SUSY multiplet.

- Also, the coupling $g_{\mathrm{A}}=\sqrt{2 \alpha^{\prime}} g_{\mathrm{YM}}$ of vertex operators is suppressed, i.e. set to unity.

All these symmetry groups involve their metrics $\eta^{m n}, \eta^{\mu \nu}, \delta_{m n}^{(6)}$ as well as gamma matrices and charge conjugation matrices as Clebsch-Gordan coefficients:

- $\gamma_{\alpha \dot{\beta}}^{m}, \bar{\gamma}_{m}^{\dot{\alpha} \beta}$ and $C_{\alpha}^{\dot{\beta}}, C^{\dot{\alpha}}{ }_{\beta}$ in $D=10$

- $\sigma_{a \dot{b}}^{\mu}, \bar{\sigma}_{\mu}^{\dot{a} b}$ and $\varepsilon_{a b}, \varepsilon^{\dot{a} \dot{b}}$ in $D=4$

- $\gamma_{m}^{I \bar{J}}, \bar{\gamma}_{\bar{I} J}^{m}$ and $C^{I}{ }_{\bar{J}}, C_{\bar{I}}^{J}$ for the internal $S O(6)$ of $\mathcal{N}=4$ SUSY

- standard Pauli matrices $\tau_{A}{ }^{i}{ }_{j}$ and $\varepsilon^{i j}$ for the $S U(2)$ R-symmetry of $\mathcal{N}=2$ SUSY

Our conventions for the slash notation is

$$
\begin{array}{lll}
\not k_{\alpha \dot{\beta}}=k_{m} \gamma_{\alpha \dot{\beta}}^{m}, & \not k^{\dot{\beta} \alpha}=k^{m} \bar{\gamma}_{m}^{\dot{\beta} \alpha} & \text { in } D=10 \\
\not k a \dot{b}=k_{\mu} \sigma_{a \dot{b}}^{\mu}, & \not k^{\dot{b} a}=k^{\mu} \bar{\sigma}_{\mu}^{\dot{b} a} & \text { in } D=4
\end{array}
$$

The totally antisymmetric $\varepsilon$ tensors are normalized to having nonzero \pm 1 , e.g. $\varepsilon^{\mu \nu \lambda \rho}$ for $D=4$ vectors and $\varepsilon_{A B C}$ for the adjoint representation of $S U(2)$.

The signature of the Dirac algebras is negative in lines with the Wess \& Bagger conventions:

$$
\begin{aligned}
\gamma_{\alpha \dot{\beta}}^{m} \bar{\gamma}^{n \dot{\beta} \gamma}+\gamma_{\alpha \dot{\beta}}^{n} \bar{\gamma}^{m \dot{\beta} \gamma} & =-2 \eta^{m n} \delta_{\alpha}^{\gamma} \\
\sigma_{a \dot{b}}^{\mu} \bar{\sigma}^{\nu \dot{b} c}+\sigma_{a \dot{b}}^{\nu} \bar{\sigma}^{\mu \dot{b c}} & =-2 \eta^{\mu \nu} \delta_{a}^{c} \\
\gamma_{m}^{I \bar{J}} \bar{\gamma}_{n \bar{J} K}+\gamma_{n}^{I \bar{J}} \bar{\gamma}_{m \bar{J} K} & =-2 \delta_{m n}^{(6)} \delta_{K}^{I}
\end{aligned}
$$

On the other hand, the $S U(2)$ Pauli matrices obey the multiplication rule

$$
\left(\tau_{A}\right)^{i}{ }_{j}\left(\tau_{B}\right)^{j}{ }_{k}=\delta_{A B} \delta_{k}^{i}+i \varepsilon_{A B C}\left(\tau^{C}\right)^{i}{ }_{k}
$$

Useful material on spinors in various spacetime dimensions can be found in [37, 38, 39], the present conventions closely follow [18, 19, 20].

\section{B Operator product expansions}

This appendix gathers the operator product expansions needed to evaluate the BRST constraints and SUSY variations. Before taking a closer look at the interacting SCFTs, let us display the free 
field OPEs for the sake of completeness, namely

$$
\begin{aligned}
i \partial X^{\mu}(z) \mathrm{e}^{i k \cdot X}(w) & \sim\left[\frac{2 \alpha^{\prime} k^{\mu}}{z-w}+i \partial X^{\mu}(w)+(z-w) i \partial^{2} X^{\mu}(w)+\ldots\right] \mathrm{e}^{i k \cdot X}(w) \\
i \partial X^{\mu}(z) i \partial X^{\nu}(w) \mathrm{e}^{i k \cdot X}(w) & \sim\left[\frac{2 \alpha^{\prime} \eta^{\mu \nu}}{(z-w)^{2}}+\frac{2 \alpha^{\prime} k^{\mu} i \partial X^{\nu}(w)}{z-w}+i \partial X^{\mu} i \partial X^{\nu}(w)+\ldots\right] \mathrm{e}^{i k \cdot X}(w)
\end{aligned}
$$

as well as

$$
\psi^{\mu}(z) \psi^{\nu}(w) \sim \frac{\eta^{\mu \nu}}{z-w}+\psi^{\mu} \psi^{\nu}(w)+(z-w) \partial \psi^{\mu} \psi^{\nu}(w)+\ldots
$$

They are valid in any number of compactification dimensions. Another universal feature is the superghost CFT, governed by

$$
\begin{aligned}
\mathrm{e}^{q_{1} \phi(z)} \mathrm{e}^{q_{2} \phi(w)}=(z & -w)^{-q_{1} q_{2}}\left[\mathrm{e}^{\left(q_{1}+q_{2}\right) \phi(w)}+q_{1}(z-w) \partial \phi \mathrm{e}^{\left(q_{1}+q_{2}\right) \phi(w)}\right. \\
& \left.+\frac{1}{2}(z-w)^{2}\left[q_{1} \partial^{2} \phi+q_{1}^{2}(\partial \phi)^{2}\right] \mathrm{e}^{\left(q_{1}+q_{2}\right) \phi(w)}+\ldots\right] .
\end{aligned}
$$

The following subsections consider the interacting RNS CFT of the $\psi$ fermion and its spin fields $S$ as well as its excited versions. The OPEs were pioneered in [17] and can be checked by means of correlation functions gathered in [18, 19, 20], and a broader discussion of the RNS operator algebra in various dimensions will be given in [21].

\section{B.1 Spacetime CFT in $D=10$}

Evaluating the BRST conditions on the most general fermion vertex operator at the first mass level requires OPEs

$$
\begin{aligned}
\psi^{m}(z) S_{\alpha}(w) & \sim \frac{\gamma_{\alpha \dot{\beta}}^{m} S^{\dot{\beta}}(w)}{\sqrt{2}(z-w)^{1 / 2}}+(z-w)^{1 / 2}\left[S_{\alpha}^{m}(w)+\frac{2 \gamma_{\alpha \dot{\beta}}^{m}}{\sqrt{2} 5} \partial S^{\dot{\beta}}(w)\right]+\ldots \\
\psi^{m}(z) \partial S_{\alpha}(w) & \sim \frac{\gamma_{\alpha \dot{\beta}}^{m} S^{\dot{\beta}}(w)}{2 \sqrt{2}(z-w)^{3 / 2}}-\frac{S_{\alpha}^{m}(w)}{2(z-w)^{1 / 2}}+\frac{4 \gamma_{\alpha \dot{\beta}}^{m} \partial S^{\dot{\beta}}(w)}{5 \sqrt{2}(z-w)^{1 / 2}}+\ldots \\
\psi_{m}(z) S_{n}^{\dot{\beta}}(w) & \sim \frac{\eta_{m n} S^{\dot{\beta}}(w)}{(z-w)^{3 / 2}}+\frac{\bar{\gamma}_{m}^{\dot{\beta} \alpha} S_{n \alpha}(w)}{\sqrt{2}(z-w)^{1 / 2}}-\frac{2 \eta_{m n} \partial S^{\dot{\beta}}(w)}{5(z-w)^{1 / 2}}+\ldots
\end{aligned}
$$

in $D=10$. The corresponding SUSY variations are computed by means of

$$
S^{\dot{\beta}}(z) \psi_{m}(w) \sim \frac{\bar{\gamma}_{m}^{\dot{\beta} \alpha} S_{\alpha}(w)}{\sqrt{2}(z-w)^{1 / 2}}+(z-w)^{1 / 2}\left[S_{m}^{\dot{\beta}}(w)+\frac{3 \bar{\gamma}_{m}^{\dot{\beta} \alpha} \partial S_{\alpha}(w)}{5 \sqrt{2}}\right]+\ldots
$$




$$
\begin{aligned}
& S^{\dot{\beta}}(z) \psi_{m} \psi_{n} \psi_{p}(w) \sim \frac{-1}{2 \sqrt{2}(z-w)^{3 / 2}} \bar{\gamma}_{m n p}^{\dot{\beta} \alpha} S_{\alpha}(w)-\frac{3}{2(z-w)^{1 / 2}}\left(\bar{\gamma}_{[m n}\right)^{\dot{\beta}} S_{p]}^{\dot{\alpha}}(w) \\
& \quad+\frac{1}{10 \sqrt{2}(z-w)^{1 / 2}} \bar{\gamma}_{m n p}^{\dot{\beta} \alpha} \partial S_{\alpha}(w)+\ldots \\
& S^{\dot{\beta}}(z) \partial \psi_{m}(w) \sim \frac{\bar{\gamma}_{m}^{\dot{\beta} \alpha} S_{\alpha}(w)}{2 \sqrt{2}(z-w)^{3 / 2}}-\frac{S_{m}^{\dot{\beta}}(w)}{2(z-w)^{1 / 2}}+\frac{7 \bar{\gamma}_{m}^{\dot{\beta} \alpha} \partial S_{\alpha}(w)}{10 \sqrt{2}(z-w)^{1 / 2}}+\ldots
\end{aligned}
$$

for the NS sector and

$$
\begin{aligned}
& S_{\alpha}(z) S_{\beta}(w) \sim \frac{\left(\gamma^{m} C\right)_{\alpha \beta} \psi_{m}(w)}{\sqrt{2}(z-w)^{3 / 4}}+(z-w)^{1 / 4} \frac{\left(\gamma^{m} C\right)_{\alpha \beta} \partial \psi_{m}(w)}{2 \sqrt{2}} \\
&-(z-w)^{1 / 4} \frac{\left(\gamma^{m n p} C\right)_{\alpha \beta} \psi_{m} \psi_{n} \psi_{p}(w)}{12 \sqrt{2}}+\ldots \\
& S_{\alpha}(z) S_{m}^{\dot{\beta}}(w) \sim \frac{C_{\alpha}^{\dot{\beta}} \psi_{m}(w)}{(z-w)^{7 / 4}}-\frac{C_{\alpha} \dot{\beta} \partial \psi_{m}(w)}{2(z-w)^{3 / 4}}-\frac{\left(\gamma^{n p} C\right)_{\alpha} \dot{\beta} \psi_{m} \psi_{n} \psi_{p}(w)}{4(z-w)^{3 / 4}}+\ldots \\
& S_{\alpha}(z) \partial S_{\beta}(w) \sim \frac{3\left(\gamma^{m} C\right)_{\alpha \beta} \psi_{m}(w)}{4 \sqrt{2}(z-w)^{7 / 4}}+\frac{7\left(\gamma^{m} C\right)_{\alpha \beta} \partial \psi_{m}(w)}{8 \sqrt{2}(z-w)^{3 / 4}} \\
&+\frac{\left(\gamma^{m n p} C\right)_{\alpha \beta} \psi_{m} \psi_{n} \psi_{p}(w)}{48 \sqrt{2}(z-w)^{3 / 4}}+\ldots
\end{aligned}
$$

for the $\mathrm{R}$ sector.

\section{B.2 Spacetime CFT in $D=4$}

In $D=4$ spacetime dimensions, $h=\frac{1}{4}$ spin fields $S_{a}, S^{\dot{b}}$ of both chiralities are present. The OPEs between spinors and vectors or $p$-forms treat both chiralities on equal footing, e.g.

$$
\begin{aligned}
\psi^{\mu}(z) S_{a}(w) & \sim \frac{\sigma_{a \dot{b}}^{\mu} S^{\dot{b}}(w)}{\sqrt{2}(z-w)^{1 / 2}}+(z-w)^{1 / 2}\left[S_{a}^{\mu}(w)+\frac{\sigma_{a \dot{b}}^{\mu}}{\sqrt{2}} \partial S^{\dot{b}}(w)\right]+\ldots \\
\psi_{\mu}(z) S^{\dot{b}}(w) & \sim \frac{\bar{\sigma}_{\mu}^{\dot{b} a} S_{a}(w)}{\sqrt{2}(z-w)^{1 / 2}}+(z-w)^{1 / 2}\left[S_{\mu}^{\dot{b}}(w)+\frac{\bar{\sigma}_{\mu}^{\dot{b} a}}{\sqrt{2}} \partial S_{a}(w)\right]+\ldots
\end{aligned}
$$

that is why we only display one chiral half of further OPEs:

$$
\begin{aligned}
\psi^{\mu}(z) \partial S_{a}(w) & \sim \frac{\sigma_{a \dot{b}}^{\mu} S^{\dot{b}}(w)}{2 \sqrt{2}(z-w)^{3 / 2}}-\frac{S_{a}^{\mu}(w)}{2(z-w)^{1 / 2}}+\frac{\sigma_{a \dot{b}}^{\mu} \partial S^{\dot{b}}(w)}{2 \sqrt{2}(z-w)^{1 / 2}}+\ldots \\
\psi_{\mu}(z) S_{\nu}^{\dot{b}}(w) & \sim \frac{\eta_{\mu \nu} S^{\dot{b}}(w)}{(z-w)^{3 / 2}}+\frac{\bar{\sigma}_{\mu}^{\dot{b} a} S_{\nu a}(w)}{\sqrt{2}(z-w)^{1 / 2}}-\frac{\eta_{\mu \nu} \partial S^{\dot{b}}(w)}{(z-w)^{1 / 2}}+\ldots
\end{aligned}
$$

Four-dimensional SUSY variations of NS operators require

$$
S^{\dot{b}}(z) \psi_{\mu}(w) \sim \frac{\bar{\sigma}_{\mu}^{\dot{b} a} S_{a}(w)}{\sqrt{2}(z-w)^{1 / 2}}+(z-w)^{1 / 2} S_{\mu}^{\dot{b}}(w)+\ldots
$$




$$
\begin{aligned}
S^{\dot{b}}(z) \psi_{\mu} \psi_{\nu}(w) & \sim \frac{-\left(\bar{\sigma}_{\mu \nu}\right)_{\dot{a}}^{\dot{b}} S^{\dot{a}}(w)}{2(z-w)}+\sqrt{2} \bar{\sigma}^{[\nu \mid \dot{b} a} S_{a}^{\mid \mu]}(w)+\frac{1}{2}\left(\bar{\sigma}_{\mu \nu}\right)^{\dot{b}} \dot{a} \partial S^{\dot{a}}(w)+\ldots \\
S^{\dot{b}}(z) \psi_{\mu} \psi_{\nu} \psi_{\lambda}(w) & \sim \frac{-1}{2 \sqrt{2}(z-w)^{3 / 2}} \bar{\sigma}_{\mu \nu \lambda}^{\dot{b} a} S_{a}(w)-\frac{3}{2(z-w)^{1 / 2}}\left(\bar{\sigma}_{[\mu \nu}\right)_{\dot{a}}^{\dot{b}} S_{\lambda]}^{\dot{a}}(w) \\
& +\frac{1}{\sqrt{2}(z-w)^{1 / 2}} \bar{\sigma}_{\mu \nu \lambda}^{\dot{b} a} \partial S_{a}(w)+\ldots \\
S^{\dot{b}}(z) \partial \psi_{\mu}(w) & \sim \frac{\bar{\sigma}_{\mu}^{\dot{b} a} S_{a}(w)}{2 \sqrt{2}(z-w)^{3 / 2}}-\frac{S_{\mu}^{\dot{b}}(w)}{2(z-w)^{1 / 2}}+\frac{\bar{\sigma}_{\mu}^{\dot{b} a} \partial S_{a}(w)}{\sqrt{2}(z-w)^{1 / 2}}+\ldots
\end{aligned}
$$

With two $\mathrm{R}$ sector states involved, the OPEs are sensitive to their relative chirality:

$$
\begin{aligned}
& S_{a}(z) S_{b}(w) \sim \frac{\varepsilon_{a b}}{(z-w)^{1 / 2}}-\frac{1}{4}(z-w)^{1 / 2}\left(\sigma^{\mu \nu} \varepsilon\right)_{a b} \psi_{\mu} \psi_{\nu}(w)+\ldots \\
& S_{a}(z) S^{\dot{b}}(w) \sim \frac{\left(\sigma^{\mu} \varepsilon\right)_{a}{ }^{\dot{b}} \psi_{\mu}(w)}{\sqrt{2}}+(z-w) \frac{\left(\sigma^{\mu} \varepsilon\right)_{a}{ }^{\dot{b}} \partial \psi_{\mu}(w)}{2 \sqrt{2}} \\
& -(z-w) \frac{\left(\sigma^{\mu \nu \lambda} \varepsilon\right)_{a}^{b} \psi_{\mu} \psi_{\nu} \psi_{\lambda}(w)}{12 \sqrt{2}}+\ldots \\
& S_{a}(z) S_{\mu}^{\dot{b}}(w) \sim \frac{\left(\sigma^{\nu} \varepsilon\right)_{a} \dot{b} \psi_{\mu} \psi_{\nu}(w)}{\sqrt{2}(z-w)^{1 / 2}}+\ldots \\
& S_{a}(z) S_{b}^{\mu}(w) \sim \frac{\varepsilon_{a b} \psi^{\mu}(w)}{(z-w)}-\frac{\varepsilon_{a b} \partial \psi^{\mu}(w)}{2}-\frac{\left(\sigma_{\nu \lambda} \varepsilon\right)_{a b} \psi^{\mu} \psi^{\nu} \psi^{\lambda}(w)}{4}+\ldots \\
& S_{a}(z) \partial S_{b}(w) \sim \frac{\varepsilon_{a b}}{2(z-w)^{3 / 2}}+\frac{\left(\sigma^{\mu \nu} \varepsilon\right)_{a b} \psi_{\mu} \psi_{\nu}(w)}{8(z-w)^{1 / 2}}+\ldots \\
& S_{a}(z) \partial S^{\dot{b}}(w) \sim \frac{\left(\sigma^{\mu} \varepsilon\right)_{a}{ }^{\dot{b}} \partial \psi_{\mu}(w)}{2 \sqrt{2}}+\frac{\left(\sigma^{\mu \nu \lambda} \varepsilon\right)_{a}{ }^{\dot{b}} \psi_{\mu} \psi_{\nu} \psi_{\lambda}(w)}{12 \sqrt{2}}+\ldots
\end{aligned}
$$

\section{B.3 Internal CFT for $\mathcal{N}=4$ SUSY}

The internal components of the ten-dimensional NS fermion are denoted by $\Psi_{m}$ with vector index $m$ for the $S O(6)$ R-symmetry. Accordingly, the associated $h=\frac{3}{8}$ spin fields $\Sigma^{I}, \bar{\Sigma}_{\bar{J}}$ have $S O(6)$ spinor indices $I, \bar{J}=1,2,3,4$. Their mutual OPEs can be covariantly expressed in terms of $S O(6)$ gamma matrices:

$$
\begin{aligned}
\Psi_{m}(z) \Sigma^{I}(w) & \sim \frac{\gamma_{m}^{I \bar{J}} \bar{\Sigma}_{\bar{J}}(w)}{\sqrt{2}(z-w)^{1 / 2}}+(z-w)^{1 / 2}\left[\Sigma_{m}^{I}(w)+\frac{2 \gamma_{m}^{I \bar{J}}}{3 \sqrt{2}} \partial \bar{\Sigma}_{\bar{J}}(w)\right]+\ldots \\
\Psi^{m}(z) \bar{\Sigma}_{\bar{J}}^{n}(w) & \sim \frac{\delta_{(6)}^{m n} \bar{\Sigma}_{\bar{J}}(w)}{(z-w)^{3 / 2}}+\frac{\bar{\gamma}_{\bar{J} I}^{m} \Sigma^{n, I}(w)}{\sqrt{2}(z-w)^{1 / 2}}-\frac{2 \delta_{(6)}^{m n} \partial \bar{\Sigma}_{\bar{J}}(w)}{3(z-w)^{1 / 2}}+\ldots \\
\Psi_{m}(z) \partial \Sigma^{I}(w) & \sim \frac{\gamma_{m}^{I \bar{J}} \bar{\Sigma}_{\bar{J}}(w)}{2 \sqrt{2}(z-w)^{3 / 2}}-\frac{\Sigma_{m}^{I}(w)}{2(z-w)^{1 / 2}}+\frac{2 \gamma_{m}^{I \bar{J}} \partial \bar{\Sigma}_{\bar{J}}(w)}{3 \sqrt{2}(z-w)^{1 / 2}}+\ldots
\end{aligned}
$$


We need the following OPEs for computing SUSY transformations of bosons:

$$
\begin{aligned}
\bar{\Sigma}_{\bar{J}}(z) \Psi^{m}(w) & \sim \frac{\bar{\gamma}_{\bar{J} I}^{m} \Sigma^{I}(w)}{\sqrt{2}(z-w)^{1 / 2}}+(z-w)^{1 / 2}\left[\bar{\Sigma}_{\bar{J}}^{m}(w)+\frac{\bar{\gamma}_{\bar{J} I}^{m}}{3 \sqrt{2}} \partial \Sigma^{I}(w)\right]+\ldots \\
\bar{\Sigma}_{\bar{J}}(z) \Psi^{m} \Psi^{n}(w) & \sim \frac{-\left(\bar{\gamma}^{m n}\right)_{\bar{J}}^{\bar{I}} \bar{\Sigma}_{\bar{I}}(w)}{2(z-w)}+\sqrt{2} \bar{\gamma}^{[n \mid \bar{J} I} \Sigma_{I}^{m]}(w)+\frac{1}{6}\left(\bar{\gamma}^{m n}\right)_{\bar{J}}^{\bar{I}} \partial \bar{\Sigma}_{\bar{I}}(w)+\ldots \\
\bar{\Sigma}_{\bar{J}}(z) \Psi^{m} \Psi^{n} \Psi^{p}(w) & \sim \frac{-1}{2 \sqrt{2}(z-w)^{3 / 2}} \bar{\gamma}_{\bar{J} I}^{m n p} \Sigma^{I}(w)-\frac{3}{2(z-w)^{1 / 2}}\left(\bar{\gamma}^{[m n}\right)_{\bar{J}}^{\bar{I}} \bar{\Sigma}_{\bar{I}}^{p]}(w) \\
\bar{\Sigma}_{\bar{J}}(z) \partial \Psi^{m}(w) & \sim \frac{1}{2 \sqrt{2}(z-w)^{1 / 2}} \bar{\gamma}_{\bar{J} I}^{m n p} \partial \Sigma^{I}(w)+\ldots \\
2 \sqrt{2}(z-w)^{3 / 2} & -\frac{\bar{\Sigma}_{I}^{m}(w)}{2(z-w)^{1 / 2}}+\frac{5 \bar{\gamma}_{\bar{J} I}^{k} \partial \Sigma^{I}(w)}{6 \sqrt{2}(z-w)^{1 / 2}}+\ldots
\end{aligned}
$$

Again, OPEs between $\mathrm{R}$ sector states depend on the relative chirality:

$$
\begin{aligned}
& \Sigma^{I}(z) \bar{\Sigma}_{\bar{J}}(w) \sim \frac{C^{I} \bar{J}^{3 / 4}-\frac{1}{4}(z-w)^{1 / 4}\left(\gamma_{m n} C\right)^{I} \Psi_{\bar{J}}^{m} \Psi^{n}(w)+\ldots}{(z-w)^{3 J} \Psi^{m}(w)}+(z-w)^{3 / 4} \frac{\left(\gamma_{m} C\right)^{I J} \partial \Psi^{m}(w)}{2 \sqrt{2}} \\
& \Sigma^{I}(z) \Sigma^{J}(w) \sim \frac{\left(\gamma_{m} C\right)^{I J}}{\sqrt{2}(z-w)^{1 / 4}}+(z-w)^{3 / 4} \frac{\left(\gamma_{m n p} C\right)^{I J} \Psi^{m} \Psi^{n} \Psi^{p}(w)}{12 \sqrt{2}}+\ldots \\
& \Sigma^{I}(z) \Sigma_{m}^{J}(w) \sim \frac{\left(\gamma^{n} C\right)^{I J} \Psi_{m} \Psi_{n}(w)}{\sqrt{2}(z-w)^{3 / 4}+\ldots}+\ldots \\
& \Sigma^{I}(z) \bar{\Sigma}_{\bar{J}}^{m}(w) \sim \frac{C^{I} \Psi^{m}(w)}{(z-w)^{5 / 4}-\frac{C^{I}{ }_{\bar{J}} \partial \Psi^{m}(w)}{2(z-w)^{1 / 4}}-\frac{\left(\gamma_{n p} C\right)^{I} \Psi^{m} \Psi^{n} \Psi^{p}(w)}{4(z-w)^{1 / 4}}+\ldots} \\
& \Sigma^{I}(z) \partial \bar{\Sigma}_{\bar{J}}(w) \sim \frac{3 C^{I} \bar{J}}{4(z-w)^{7 / 4}}+\frac{\left(\gamma_{m n} C\right)^{I} \Psi^{m} \Psi^{n}(w)}{16(z-w)^{3 / 4}}+\ldots \\
& \Sigma^{I}(z) \partial \Sigma^{J}(w) \sim \frac{\left(\gamma_{m} C\right)^{I J} \Psi^{m}(w)}{4 \sqrt{2}(z-w)^{5 / 4}+\frac{5\left(\gamma_{m} C\right)^{I J} \partial \Psi^{m}(w)}{8 \sqrt{2}(z-w)^{1 / 4}}} \\
&+\frac{\left(\gamma_{m n p} C\right)^{I J} \Psi^{m} \Psi^{n} \Psi^{p}(w)}{16 \sqrt{2}(z-w)^{1 / 4}}+\ldots
\end{aligned}
$$

\section{B.4 Internal CFT for $\mathcal{N}=1$ SUSY}

Most of the OPEs relevant for the internal $c=9$ SCFT described in subsection 3.4 can be derived from the CFT of a free boson:

$$
\begin{aligned}
i \partial H(z) \mathrm{e}^{i q H}(w) & \sim\left[\frac{q}{z-w}+i \partial H(w)+\ldots\right] \mathrm{e}^{i q H}(w) \\
\mathrm{e}^{i q H}(z) i \partial H(w) & \sim\left[\frac{q}{z-w}+\left(q^{2}-1\right) i \partial H(w)+\ldots\right] \mathrm{e}^{i q H}(w)
\end{aligned}
$$




$$
\mathrm{e}^{i q_{1} H}(z) \mathrm{e}^{i q_{2} H}(w) \sim(z-w)^{q_{1} q_{2}}\left[1+q_{1}(z-w) i \partial H+\ldots\right] \mathrm{e}^{i\left(q_{1}+q_{2}\right) H}(w)
$$

This allows to reproduce 3.28 and $(3.34)$ from the bosonized representations 3.35 of the operators $\mathcal{J}, \Sigma^{ \pm}$and $\mathcal{O}^{ \pm}$. Moreover, we have

$$
\begin{aligned}
\Sigma^{ \pm}(z) \mathcal{J}(w) & \sim \frac{ \pm \sqrt{3} \Sigma^{ \pm}(w)}{2(z-w)} \mp \frac{\partial \Sigma^{ \pm}(w)}{2 \sqrt{3}}+\ldots \\
\Sigma^{ \pm}(z) \mathcal{O}^{\mp}(w) & \sim(z-w)^{-3 / 2} \Sigma^{\mp}(w)-(z-w)^{-1 / 2} \partial \Sigma^{\mp}(w)+\ldots
\end{aligned}
$$

The excited spin fields $\tilde{\Sigma}^{ \pm}=g^{\mp} \mathrm{e}^{ \pm i H / \sqrt{12}}$ are canonically normalized

$$
\begin{aligned}
& \tilde{\Sigma}^{ \pm}(z) \tilde{\Sigma}^{\mp}(w) \sim \frac{1}{(z-w)^{11 / 4}} \pm \frac{i \partial H(w)}{2 \sqrt{3}(z-w)^{7 / 4}}+\ldots \\
& \tilde{\Sigma}^{ \pm}(z) \tilde{\Sigma}^{ \pm}(w) \sim \frac{g^{\mp} g^{\mp} \mathrm{e}^{ \pm \frac{i H}{\sqrt{3}}}(w)}{(z-w)^{1 / 4}}+\ldots
\end{aligned}
$$

such that the mutual singularities between standard and excited spin fields are given by

$$
\begin{aligned}
& \tilde{\Sigma}^{ \pm}(z) \Sigma^{ \pm}(w) \sim(z-w)^{1 / 4} g^{\mp} \mathrm{e}^{ \pm \frac{2 i}{\sqrt{3}} H}(w)+\ldots \\
& \tilde{\Sigma}^{ \pm}(z) \Sigma^{\mp}(w) \sim \sqrt{\frac{2}{3}} \frac{G_{\text {int }}^{\mp}(w)}{(z-w)^{1 / 4}}+\ldots
\end{aligned}
$$

Moreover, in presence of the internal supercurrents $G_{\text {int }}^{ \pm}=\sqrt{\frac{3}{2}} \mathrm{e}^{ \pm i H / \sqrt{3}} g^{ \pm}$,

$$
\begin{aligned}
G_{\text {int }}^{ \pm}(z) \tilde{\Sigma}^{ \pm}(w) & \sim \sqrt{\frac{3}{2}} \frac{\Sigma^{ \pm}(w)}{(z-w)^{5 / 2}}+\sqrt{\frac{2}{3}} \frac{\partial \Sigma^{ \pm}(w)}{(z-w)^{3 / 2}}+\ldots \\
G_{\text {int }}^{ \pm}(z) \tilde{\Sigma}^{\mp}(w) & \sim \sqrt{\frac{3}{2} \frac{g^{ \pm} g^{ \pm} \mathrm{e}^{ \pm \frac{i}{2 \sqrt{3}} H}(w)}{(z-w)^{1 / 2}}}+\ldots \\
\tilde{\Sigma}^{ \pm}(z) \mathcal{J}(w) & \sim \pm \frac{\tilde{\Sigma}^{ \pm}(w)}{2 \sqrt{3}(z-w)}+\ldots
\end{aligned}
$$

\section{Spinor helicity methods for massive wavefunctions}

Before we proceed to introduce the massive version of the spinor helicity formalism, we will make a short review for the helicity formalism of massless spinors. For massless spin- $\frac{1}{2}$ spinors, we use the following notations,

$$
\begin{aligned}
& |i\rangle=\left|k_{i}\right\rangle=u_{+}\left(k_{i}\right)=v_{-}\left(k_{i}\right)=\left(\begin{array}{c}
0 \\
k_{i}^{* \dot{a}}
\end{array}\right), \\
& \left.\mid i]=\mid k_{i}\right]=u_{-}\left(k_{i}\right)=v_{+}\left(k_{i}\right)=\left(\begin{array}{c}
k_{i, a} \\
0
\end{array}\right),
\end{aligned}
$$




$$
\begin{aligned}
& {\left[i \mid=\left[k_{i} \mid=\bar{u}_{+}\left(k_{i}\right)=\bar{v}_{-}\left(k_{i}\right)=\left(k_{i}^{a}, 0\right),\right.\right.} \\
& \langle i|=\left\langle k_{i}\right|=\bar{u}_{-}\left(k_{i}\right)=\bar{v}_{+}\left(k_{i}\right)=\left(0, k_{i, \dot{a}}^{*}\right) .
\end{aligned}
$$

Here the momentums with spinor indices denote two component commutative spinors. They are defined by

$$
\begin{gathered}
P^{\dot{a} a}=p_{\mu} \bar{\sigma}^{\mu \dot{a} a}=-p^{* \dot{a}} p^{a}, \\
P_{a \dot{a}}=p_{\mu} \sigma_{a \dot{a}}^{\mu}=-p_{a} p_{\dot{a}}^{*},
\end{gathered}
$$

where $p^{* \dot{a}}=\left(p^{a}\right)^{*}$ and $p_{\dot{a}}^{*}=\left(p_{a}\right)^{*}$. Spinor indices could be raised (lowered) by $\varepsilon^{a b}\left(\varepsilon_{a b}\right)$ or $a, b$ with dots,

$$
p^{a}=\varepsilon^{a b} p_{b}, \quad p^{* \dot{a}}=\varepsilon^{\dot{a} \dot{b}} p_{\dot{b}}^{*} .
$$

Then we can define the notations for the spinor products,

$$
\begin{gathered}
\langle p q\rangle=\langle p \mid q\rangle=\bar{u}_{-}(p) u_{+}(q)=p_{\dot{a}}^{*} q^{* \dot{a}} \\
{[p q]=[p \mid q]=\bar{u}_{+}(p) u_{-}(q)=p^{a} q_{a}}
\end{gathered}
$$

so that simply we have

$$
\begin{aligned}
{[p q] } & =-[q p], & \langle p q\rangle & =-\langle q p\rangle, \\
\langle p q\rangle^{*} & =-[p q], & \langle p p\rangle & =[p p]=0,
\end{aligned}
$$

and

$$
\langle p q\rangle[q p]=-2(p \cdot q) .
$$

\section{C.1 Massive spin one boson}

A spin $J$ particle contains $2 J+1$ spin degrees of freedom associated to the eigenstates of $J_{z}$. The choice of the quantization axis $z$ can be handled in an elegant way by decomposing the momentum $k$ into two arbitrary light-like reference momenta $p$ and $q$ :

$$
k^{\mu}=p^{\mu}+q^{\mu}, \quad k^{2}=-m^{2}=2 p q, \quad p^{2}=q^{2}=0 .
$$

Then the spin quantization axis is chosen to be the direction of $q$ in the rest frame. The $2 J+1$ spin wavefunctions depend of $p$ and $q$, however this dependence drops out in the amplitudes summed over all spin directions and in "unpolarized" cross sections. 
The massive spin one wavefunctions $\xi_{\mu}$ (transverse, i.e., $\xi_{\mu} k^{\mu}=0$ ) are given by the following polarization vectors [22, 23], up to a phase factor,

$$
\begin{aligned}
\xi_{+}^{\mu}(k) & =\frac{1}{\sqrt{2} m} p_{\dot{a}}^{*} \bar{\sigma}^{\mu \dot{a} a} q_{a}, \\
\xi_{0}^{\mu}(k) & =\frac{1}{2 m} \bar{\sigma}^{\mu \dot{a} a}\left(p_{\dot{a}}^{*} p_{a}-q_{\dot{a}}^{*} q_{a}\right), \\
\xi_{-}^{\mu}(k) & =-\frac{1}{\sqrt{2} m} q_{\dot{a}}^{*} \bar{\sigma}^{\mu \dot{a} a} p_{a} .
\end{aligned}
$$

\section{C.2 Massive spin two boson}

The massive spin two boson $\alpha^{\mu \nu}$ satisfies the following conditions,

$$
\begin{gathered}
\alpha^{\mu \nu}(k, \lambda)=\alpha^{\nu \mu}(k, \lambda), \\
k_{\mu} \alpha^{\mu \nu}(k, \lambda)=0, \\
g_{\mu \nu} \alpha^{\mu \nu}(k, \lambda)=0,
\end{gathered}
$$

where $\lambda$ expresses the helicity of $\alpha^{\mu \nu}$. We do the same decomposition of the momentum, and the wavefunction of a spin two boson can be written as [22],

$$
\begin{aligned}
\alpha^{\mu \nu}(k,+2) & =\frac{1}{2 m^{2}} \bar{\sigma}^{\mu \dot{a} a} \bar{\sigma}^{\nu \dot{b b}} p_{\dot{a}}^{*} q_{a} p_{\dot{b}}^{*} q_{b}, \\
\alpha^{\mu \nu}(k,+1) & =\frac{1}{4 m^{2}} \bar{\sigma}^{\mu \dot{a} a} \bar{\sigma}^{\nu \dot{b} b}\left[\left(p_{\dot{a}}^{*} p_{a}-q_{\dot{a}}^{*} q_{a}\right) p_{\dot{b}}^{*} q_{b}+p_{\dot{a}}^{*} q_{a}\left(p_{\dot{b}}^{*} p_{b}-q_{\dot{b}}^{*} q_{b}\right)\right], \\
\alpha^{\mu \nu}(k, 0) & =\frac{1}{2 \sqrt{6} m^{2}} \bar{\sigma}^{\mu \dot{a} a} \bar{\sigma}^{\nu \dot{b} b}\left[\left(p_{\dot{a}}^{*} p_{a}-q_{\dot{a}}^{*} q_{a}\right)\left(p_{\dot{b}}^{*} p_{b}-q_{\dot{b}}^{*} q_{b}\right)-p_{\dot{a}}^{*} q_{a} q_{\dot{b}}^{*} p_{b}-q_{\dot{a}}^{*} p_{a} p_{\dot{b}}^{*} q_{b}\right], \\
\alpha^{\mu \nu}(k,-1) & =\frac{1}{4 m^{2}} \bar{\sigma}^{\mu \dot{a} a} \bar{\sigma}^{\nu \dot{b} b}\left[\left(q_{\dot{a}}^{*} q_{a}-p_{\dot{a}}^{*} p_{a}\right) q_{\dot{b}}^{*} p_{b}+q_{\dot{a}}^{*} p_{a}\left(q_{\dot{a}}^{*} q_{a}-p_{\dot{b}}^{*} p_{b}\right)\right], \\
\alpha^{\mu \nu}(k,-2) & =\frac{1}{2 m^{2}} \bar{\sigma}^{\mu \dot{a} a} \bar{\sigma}^{\nu \dot{b} b} q_{\dot{a}}^{*} p_{a} q_{\dot{b}}^{*} p_{b} .
\end{aligned}
$$

\section{C.3 Massive spin 1/2 fermions}

Massive spin $\frac{1}{2}$ fermions satisfy the Dirac equation,

$$
\begin{aligned}
& (\not k+m) u(k)=0, \\
& (\not k-m) v(k)=0,
\end{aligned}
$$

where $u(k)$ and $v(k)$ are positive and negative energy solutions with momentum $k^{\mu}$, which correspond to fermion and anti-fermion wavefunctions respectively. Since we do not deal with the 
wavefunctions of the negative energy solutions, we will only present $u(k)$ wavefunction here. $u(k)$ satisfies the spin-sum relations, orthogonal condition and the normalization condition,

$$
\begin{gathered}
\sum_{\text {spin }} u_{ \pm}(k) \bar{u}_{ \pm}(k)=-\not k+m \\
\bar{u}_{ \pm}(k) u_{\mp}(k)=0 \\
\bar{u}_{ \pm}(k) u_{ \pm}(k)=2 m
\end{gathered}
$$

Writing the four component spinor $u(k)$ as

$$
u=\left(\begin{array}{c}
\chi_{a} \\
\bar{\eta}^{\dot{a}}
\end{array}\right)
$$

and plugging it into the Dirac equation, we get

$$
k_{\mu}\left(\begin{array}{cc}
0 & \sigma_{a \dot{a}}^{\mu} \\
\bar{\sigma}^{\mu \dot{a} a} & 0
\end{array}\right)\left(\begin{array}{c}
\chi_{a} \\
\bar{\eta}^{\dot{a}}
\end{array}\right)=-m\left(\begin{array}{c}
\chi_{a} \\
\bar{\eta}^{\dot{a}}
\end{array}\right) .
$$

The Dirac equation is decomposed to,

$$
\begin{aligned}
k_{\mu} \bar{\sigma}^{\mu \dot{a} a} \chi_{a} & =-m \bar{\eta}^{\dot{a}}, \\
k_{\mu} \sigma_{a \dot{a}}^{\mu} \bar{\eta}^{\dot{a}} & =-m \chi_{a} .
\end{aligned}
$$

Making the same decomposition of the momentum $k^{\mu}=p^{\mu}+q^{\mu}$, we can obtain the wavefunction of the massive spin $\frac{1}{2}$ fermion [23],

$$
\begin{aligned}
& u_{+}(k)=\left(\begin{array}{c}
\frac{\langle q p\rangle}{m} q_{a} \\
p^{* \dot{a}}
\end{array}\right), \\
& u_{-}(k)=\left(\begin{array}{c}
p_{a} \\
\frac{[q p]}{m} q^{* \dot{a}}
\end{array}\right) .
\end{aligned}
$$

\section{C.4 Massive spin 3/2 fermions}

A massive spin $\frac{3}{2}$ fermion are described by a Rarita-Schwinger spinor-vector $\Psi^{A, \mu}$ which satisfies equations,

$$
\begin{aligned}
(i \not \partial-m)^{A}{ }_{B} \Psi^{B, \mu} & =0, \\
\left(\gamma_{\mu}\right)^{A}{ }_{B} \Psi^{B, \mu} & =0, \\
\partial_{\mu} \Psi^{B, \mu} & =0,
\end{aligned}
$$


where $A$ and $B$ are spinor indices. Again we only consider the positive energy solution $U$, it satisfies,

$$
\begin{aligned}
(\not k+m)^{A}{ }_{B} U(k)^{B, \mu} & =0, \\
\bar{U}_{A, \mu}(k, \lambda) U^{A, \mu}\left(k, \lambda^{\prime}\right) & =2 m \delta_{\lambda \lambda^{\prime}} .
\end{aligned}
$$

The wavefunction of $U$ can be written as [23],

$$
\begin{aligned}
U^{A, \mu}\left(+\frac{3}{2}\right) & =\frac{1}{\sqrt{2} m}\left(\begin{array}{c}
\frac{\langle q p\rangle}{m} q_{a} \\
p^{* \dot{a}}
\end{array}\right)\left(p_{\dot{b}}^{*} \bar{\sigma}^{\mu \dot{b} b} q_{b}\right) \\
U^{A, \mu}\left(+\frac{1}{2}\right) & =\frac{\bar{\sigma}^{\mu \dot{b} b}}{\sqrt{6} m}\left[\left(\begin{array}{c}
\frac{\langle q p\rangle}{m} q_{a} \\
p^{* \dot{a}}
\end{array}\right)\left(p_{\dot{b}}^{*} p_{b}-q_{\dot{b}}^{*} q_{b}\right)+\left(\begin{array}{c}
\frac{\langle q p\rangle}{m} p_{a} \\
-q^{* \dot{a}}
\end{array}\right)\left(p_{\dot{b}}^{*} q_{b}\right)\right] \\
U^{A, \mu}\left(-\frac{1}{2}\right) & =\frac{\bar{\sigma}^{\mu \dot{b} b}}{\sqrt{6} m}\left[\left(\begin{array}{c}
p_{a} \\
\frac{[q p]}{m} q^{* \dot{a}}
\end{array}\right)\left(p_{\dot{b}}^{*} p_{b}-q_{\dot{b}}^{*} q_{b}\right)+\left(\begin{array}{c}
-q_{a} \\
\frac{[q p]}{m} p^{* \dot{a}}
\end{array}\right)\left(q_{\dot{b}}^{*} p_{b}\right)\right] \\
U^{A, \mu}\left(-\frac{3}{2}\right) & =\frac{1}{\sqrt{2} m}\left(\begin{array}{c}
p_{a} \\
\frac{[q p]}{m} q^{* \dot{a}}
\end{array}\right)\left(q_{\dot{b}}^{*} \bar{\sigma}^{\mu \dot{b} b} p_{b}\right) .
\end{aligned}
$$

\section{References}

[1] W. -Z. Feng, D. Lüst, O. Schlotterer, S. Stieberger, T. R. Taylor, "Direct Production of Lightest Regge Resonances," Nucl. Phys. B843 (2011) 570-601, arXiv:1007.5254 [hep-th]].

[2] D. Amati, M. Ciafaloni and G. Veneziano, "Can Spacetime Be Probed Below the String Size?" Phys. Lett. B 216 (1989) 41.

[3] D. Lüst, S. Stieberger and T. R. Taylor, "The LHC String Hunter's Companion," Nucl. Phys. B 808 (2009) 1, arXiv:0807.3333 [hep-th]].

[4] D. Lüst, O. Schlotterer, S. Stieberger and T. R. Taylor, "The LHC String Hunter's Companion (II): Five-Particle Amplitudes and Universal Properties," Nucl. Phys. B 828 (2010) 139, arXiv:0908.0409 [hep-th]].

[5] I. G. Koh, W. Troost, A. Van Proeyen, "Covariant Higher Spin Vertex Operators In The Ramond Sector," Nucl. Phys. B292 (1987) 201.

[6] N. Berkovits and O. Chandia, "Massive Superstring Vertex Operator in D = 10 Superspace," JHEP 0208 (2002) 040 arXiv:hep-th/0204121.

[7] A. Sagnotti and M. Taronna, "String Lessons for Higher-Spin Interactions," Nucl. Phys. B 842 (2011) 299 arXiv:1006.5242 [hep-th]]. 
[8] M. Bianchi, L. Lopez, R. Richter, "On Stable Higher Spin States in Heterotic String Theories," JHEP 1103 (2011) 051, arXiv:1010.1177 [hep-th]].

[9] I. Y. Park, "Scattering of Massive Open Strings in Pure Spinor," Nucl. Phys. B 852 (2011) 287, arXiv:1101.1204 [hep-th]].

[10] W. -Z. Feng, T. R. Taylor, "Higher Level String Resonances in Four Dimensions," Nucl. Phys. B 856 (2012) 247-277, arXiv:1110.1087 [hep-th]].

[11] D. Friedan, E.J. Martinec and S.H. Shenker, "Conformal Invariance, Supersymmetry And String Theory," Nucl. Phys. B 271 (1986) 93.

[12] T. Banks, L.J. Dixon, D. Friedan and E.J. Martinec, "Phenomenology and Conformal Field Theory Or Can String Theory Predict the Weak Mixing Angle?," Nucl. Phys. B 299 (1988) 613.

[13] T. Banks and L.J. Dixon, "Constraints on String Vacua with Spacetime Supersymmetry," Nucl. Phys. B 307 (1988) 93.

[14] S. Ferrara, D. Lüst and S. Theisen, "World-sheet Versus Spectrum Symmetries In Heterotic And Type II Superstrings.," Nucl. Phys. B 325, 501 (1989).

[15] S. Stieberger and T.R. Taylor, "Supersymmetry Relations and MHV Amplitudes in Superstring Theory," Nucl. Phys. B 793, 83 (2008), arXiv:0708.0574 [hep-th]].

[16] J. Cohn, D. Friedan, Z. -a. Qiu, S. H. Shenker, "Covariant Quantization Of Supersymmetric String Theories: The Spinor Field Of The Ramond-neveu-schwarz Model," Nucl. Phys. B278 (1986) 577.

[17] V. A. Kostelecky, O. Lechtenfeld, W. Lerche, S. Samuel, S. Watamura, "Conformal Techniques, Bosonization and Tree Level String Amplitudes," Nucl. Phys. B288 (1987) 173.

[18] D. Haertl, O. Schlotterer, S. Stieberger, "Higher Point Spin Field Correlators in D=4 Superstring Theory," Nucl. Phys. B834 (2010) 163-221. arXiv:0911.5168 [hep-th]].

[19] D. Haertl, O. Schlotterer, "Higher Loop Spin Field Correlators in Various Dimensions," Nucl. Phys. B849 (2011) 364-409. arXiv:1011.1249 [hep-th]]

[20] O. Schlotterer, "Scattering Amplitudes in Open Superstring Theory," (2011) PhD thesis.

[21] O. Schlotterer, S. Theisen "The Covariant RNS Operator Algebra," work in progress.

[22] D. Spehler and S. F. Novaes, "Helicity Wave Functions for Massless and Massive Spin-2 Particles," Phys. Rev. D 44 (1991) 3990. 
[23] S. F. Novaes and D. Spehler, "Weyl-van der Waerden Spinor Technique for Spin- $\frac{3}{2}$ Fermions," Nucl. Phys., B371 (1992) 618.

[24] R. H. Boels, C. Schwinn, "On-shell Supersymmetry for Massive Multiplets," Phys. Rev. D84 (2011) 065006, arXiv:1104.2280 [hep-th]].

[25] V. P. Nair "A Current Algebra for Some Gauge Theory Amplitudes," Phys. Lett. B 214, 215 (1988).

[26] M. Bianchi, H. Elvang and D. Z. Freedman "Generating Tree Amplitudes in $N=4 \mathrm{SYM}$ and $N=8$ SG," JHEP 0809 (2008) 063, arXiv:0805.0757 [hep-th]]; J. M. Drummond, J. Henn, G. P. Korchemsky and E. Sokatchev, "Dual Superconformal Symmetry of Scattering Amplitudes in $N=4$ Super-Yang-Mills Theory," Nucl. Phys. B 828 (2010) 317-374, arXiv:0807.1095 [hep-th]]; J. M. Drummond, J. Henn, G. P. Korchemsky and E. Sokatchev, "Generalized Unitarity for $N=4$ Super-Amplitudes," arXiv:0808.0491 [hep-th]]; N. Arkani-Hamed, F. Cachazo and J. Kaplan "What is the Simplest Quantum Field Theory?" arXiv:0808.1446 [hep-th]]; H. Elvang, Y.-t. Huang and C. Peng "On-shell Superamplitudes in $N<4$ SYM," arXiv:1102.4843v1 [hep-th]].

[27] A. Hanany, D. Forcella, J. Troost, "The Covariant Perturbative String Spectrum," Nucl. Phys. B846 (2011) 212-225, arXiv:1007.2622 [hep-th]].

[28] O. Schlotterer, "Higher Spin Scattering in Superstring Theory," [arXiv:1011.1235 [hep-th]].

[29] C. R. Mafra, O. Schlotterer and S. Stieberger, "Complete N-Point Superstring Disk Amplitude I. Pure Spinor Computation," arXiv:1106.2645 [hep-th]].

[30] C. R. Mafra, O. Schlotterer and S. Stieberger, "Complete N-Point Superstring Disk Amplitude II. Amplitude and Hypergeometric Function Structure," arXiv:1106.2646 [hep-th]].

[31] R. H. Boels and D. O'Connell, "Simple Superamplitudes in Higher Dimensions," arXiv:1201.2653 [hep-th]].

[32] R. H. Boels, "Three Particle Superstring Amplitudes with Massive Legs," arXiv:1201.2655 [hep-th]].

[33] N. Berkovits, "Super Poincare Covariant Quantization of the Superstring," JHEP 0004 (2000) 018, arXiv:hep-th/0001035.

[34] N. Berkovits, "A New Description of the Superstring," In *Rio de Janeiro 1995, Particles and fields* 390-418, arXiv:hep-th/9604123]. 
[35] N. Berkovits, M. M. Leite "First Massive State of the Superstring in Superspace," Phys. Lett. B415 (1997) 144-148, arXiv:hep-th/9709148.

[36] N. Berkovits, M.M. Leite "Superspace Action for the First Massive States of the Superstring," Phys. Lett. B454 (1999) 38-42, arXiv:hep-th/9812153].

[37] P. C. West, "Supergravity, Brane Dynamics and String Duality," arXiv:hep-th/9811101.

[38] J. Polchinski, "Superstring Theory and beyond, Volume II," CUP 2005.

[39] J. A. Strathdee, "Extended Poincaré Supersymmetry," Int. J. Mod. Phys. A 2 (1987) 273. 